\title{
SHOCK DYNAMICS IN NON-UNIFORM MEDIA
}

\author{
Thesis by \\ Christopher Jeyaparan Catherasoo
}

In Partial Fulfillment

of the Requirements for the Degree of

Doctor of Philosophy

\author{
California Institute of Technology \\ Pasadena, Callifornia
}

1982

(Submitted 16 April 1982) 
- ii -

- 1982

Christopher Jeyaparan Catherasoo All Rights Reserved 
- iii -

To my parents, who after all, started me on my numbers. 


\section{ACKNOWLEDGFMENTS}

The author takes this opportunity to sincerely thank all those who have helped him in this research effort. He is indebted to Professor Bradford Sturtevant for suggesting the problem and for his invaluable guidance and support throughout the project. To his fellow graduate students, he is sincerely grateful for many stimulating discussions. His appreciation also goes to the computing centre staff, especially the consultants and operators, for their cooperation during the preparation of this manuscript. The financial support provided by the California Institute of Technology is gratefully acknowledged.

Finally, the author's deepest appreciation and special thanks go to all his friends, both here at Caltech and elsewhere, and most importantly to his parents, sisters and brother-in-law for their constant encouragement, emotional support and prayers, without which this effort would not have been possible. 


\begin{abstract}
The theory of shock dynamies in two dimensions is reformulated to treat shock propagation in a non-uniform medium. The analysis yields a system of hyperbolic equations with source terms representing the generation of disturbances on the shock wave as it propagates into the fluid non-uniformities. The theory is applied to problems involving the refraction of a plane shock wave at a free plane gaseous interface. The "slow-fast" interface is investigated in detail, while the "fast-slow" interface is treated only briefly. Intrinsic to the theory is a relationship analogous to Snell's law of refraction at an interface. The theory predicts both regular and irregular ( $\mathrm{Mach}$ ) refraction, and a criterion is developed for the transition from one to the other. Quantitative results for several different shock strengths, angles of incidence and sound speed ratios are presented. An analogy between shock refraction and the motion of a force field in unsteady one-dimensional gasdynamies is pointed out. Also discussed is the limiting case for a shock front to be continuous at the interface. Comparison of results is made with existing experimental data, with transition calculations based on three-shock theory, and with the simple case of normal interaction.
\end{abstract}




\section{TABLE OF CONTENTS}

Copyright

Dedication

Acknowledgements

iv

Abstract

$\mathrm{v}$

Table of Contents

vi

List of Figures

viii

List of Tables

$\mathrm{x}$

List of Symbols

xi

1.0 INTRODUCTION

2.0 GOVERNING EQUATIONS 6

2.1 Quasi-one-dimensional non-steady flow in a 6 non-uniform medium with finite area changes

2.2 Geometrical shock dynamics in two dimensions 14

2.3 Wave propagation on the shock 17

2.4 The characteristic system 25

3.0 SHOCK REFRACTION 31

$\begin{array}{lll}3.1 & \text { Problem } 1 & 36\end{array}$

3.2 Problem 2 43

4.0 COMPARISON WITH EXPERIMENT AND THEORY

4.1 Comparison with experimental results 50

4.2 Comparison with three-shock theory 57

4.3 Normal interaction $\quad 59$

$\begin{array}{lll}4.4 & \text { Closing remarks } & 61\end{array}$ 
- vii -

$5.0 \quad$ SUMMARY AND CONCLUSIONS

6.0 REFERENCES 65

APPENDICES

A. Integration of the ray tube area function 66

B. The coordinate transformation relations 69

C. The singularity 73

D. Shock wave interaction with a normal contact 80 discontinuity

E. Flowcharts and computer programs 90 
- viii -

\section{LIST OF FIGURES}

Figure

Title

Page

$2.1 \quad$ The $(\alpha, \beta)$ coordinate system. 15

2.2 Variation of the functions $A, \lambda, \mu, \nu, v$ and $\omega$ with 24 Mach number.

2.3 The contact surface discontinuity. 28

3.1 Configurations for Problem 1: 32

(a) Contact surface above leading $\mathrm{C}^{+}$characteristic;

(b) Contact surface between leading $C^{+}$and $C^{-}$ characteristics;

(c) Contact surface below leading $C^{-}$characteristic.

3.2 Configurations for Problem 2;

(a) Contact surface above leading $C^{+}$characteristic;

(b) Contact surface between leading $C^{+}$and wall.

3.3 Sample solutions for Problem 1 with $M_{1}=5.0$ and $a_{02} / a_{01}=2.0$;

(a) Regular refraction, $\delta_{I}=60.0^{\circ}$;

(b) Irregular refraction, $\delta_{\bar{I}}=45.0^{\circ}$;

(c) Irregular refraction, $\delta_{I}=30.0^{\circ}$;

(d) Irregular refraction, $\delta_{I}=15.0^{\circ}$;

(e) Irregular refraction, $\delta_{I}=0^{\circ}$;

(i) Regular reîraction, $\delta_{I}=-45.0^{\circ}$.

3.4 Sample solutions for Problem 2 with $M_{1}=5.0$, $a_{02} / a_{01}=2.0$ and $\delta_{W}=15.0^{\circ}$;
(a) Regular refraction, $\delta_{I}=65.0^{\circ}$;
(b) Regular refraction, $\delta_{I}=60.0^{\circ}$;
(c) Irregular refraction, $\delta_{I}=45.0^{\circ}$;
(d) Irregular refraction, $\delta_{I}=30.0^{\circ}$.

3.5 Irregular refraction solution for Problem 2 with $M_{1}=2.0, a_{02} / a_{01}=2.0, \delta_{W}=0^{\circ}$ and $\delta_{I}=30.0^{\circ}$. 
4.1 Transmitted shock wave angle $\omega_{t}$ versus incident shock wave angle $\omega_{i}$ for refraction of a plane shock at a contaminated carbon dioxide - helium interface.

4.2 Transition angles for refraction of a plane shock at a pure carbon dioxide - helium interface.

4.3 Relative error between the results from shock dynamies and the results from one-dimensional gasdynamies for normal interaction, $\delta_{I}=90^{\circ}$.

B.1 Relationship between the $(\alpha, \beta)$ and the $(x, y)$ coordinate systems.

D.1 Interaction of a shock wave with a normal contact discontinuity;
(a) Reflected shock wave;
(b) Reflected expansion wave.

E.1 Flowcharts for shock refraction calculations;
(a) Regular refraction;
(b) Irregular refraction. 
$-\mathrm{x}-$

\section{LIST OF TABLES}

Table Title Page

3.1 Summary of calculated refraction parameters for 36 Problem 1.

3.2 Summary of calculated refraction parameters for 44 Problem 2.

4.1 Calculated and measured refraction parameters 51 based on settings used by Jahn.

4.2 Calculated refraction parameters for normal interac- 59 tion. 


\section{IUST OF SYMBOLS}

Symbol

Description

Reference

a Sound speed

A Area of ray tube

Equation (2.1.26)

c Slope of characteristics in $(\alpha, \beta)$ plane

Equation (2.3.9)

C Arbitrary constant

$C^{ \pm} \quad$ Characteristics

Equation (2.3.8)

$f \quad$ Function of $M$ and $\gamma$

Equation (2.1.22)

F Simplifying notation

Equation (2.3.5)

$g \quad$ Function of $M$ and $\gamma$

Equation (2.1.23)

$G$ Simplifying notation

Equation (2.3.5)

$h \quad$ Function of $M$ and $\gamma$

Equation (2.1.24)

$J^{ \pm} \quad$ Riemarn invariants

Equation (2.4.1)

$M \quad$ Mach number

$p \quad$ Pressure

$P \quad$ Simplifying notation

Equation (2.1.15)

Q Simplifying notation

Equation (2.1.15)

$R \quad$ Simplifying notation

Equation (2.1.15)

$r \quad$ Non-dimensionalized sound speed

Equation (C.1.3)

$t \quad$ Time

u Gas velocity

$U \quad$ Shock front velocity

Equation (2.4.11)

$V \quad$ Simplifying notation

Equation (2.4.12)

$\approx \quad$ Abscissa

y Ordinate 


\begin{tabular}{|c|c|c|}
\hline$\alpha$ & Coordinate describing shock position & Figure R.1 \\
\hline$\beta$ & Coordinate describing ray position & Figure 2.1 \\
\hline$\gamma$ & Ratio of specific heats, $c_{p} / c_{v}$ & \\
\hline$\delta_{I}$ & Angle between gaseous interface and $x$-axis & Figure 2.3 \\
\hline$\delta_{W}$ & Angle between solid boundary and $x$-axis & Figure 3.2 \\
\hline$\varepsilon$ & Relative error & Equation (4.3.1) \\
\hline$\zeta$ & Function of $M$ and $\gamma$ & Equation (A.5) \\
\hline$\eta^{ \pm}$ & Angle between characteristics and $x$-axis & Equation $(2.3 .20)$ \\
\hline$\eta_{i}$ & Pressure ratio across the shock & Equation $(4.2 .1)$ \\
\hline$\theta$ & Angle between ray and $x$-axis & Figure 2.1 \\
\hline$\tilde{\theta}$ & Angle between ray and interface & Equation $(2.4 .6)$ \\
\hline$\kappa$ & Simplifying notation & Equation $(2.3 .4)$ \\
\hline$\lambda$ & Function of $M$ and $\gamma$ & Equation (2.1.21) \\
\hline$\mu$ & Function of $M$ and $\gamma$ & Equation (2.1.25) \\
\hline$\nu$ & Function of $M$ and $\gamma$ & Equation (2.3.15) \\
\hline$\phi$ & Angle between shock and $x$-axis & Figure 2.1 \\
\hline$\Phi$ & Function of $M$ and $\alpha$ & Equation (C.2.5) \\
\hline$\xi$ & Simplifying notation & Equation (2.3.4) \\
\hline$\psi$ & Function of $M$ & Equation (C.2.2) \\
\hline$\Psi$ & Function of $M$ & Equation (C.2.2) \\
\hline$\rho$ & Gas density & \\
\hline$v$ & Angle between characteristics and ray & Equation (2.3.19) \\
\hline$x$ & Angle between shock-shock and $x$-axis & Equation $(2.4 .3)$ \\
\hline$\tilde{\chi}$ & Angle between shock-shock and interface & Equation (4.1.3) \\
\hline$\omega$ & Function of $M$ and $\gamma$ & Equation $(2.3 .11)$ \\
\hline$\omega_{i}$ & Incident shock wave angle & Equation $(4.1 .1)$ \\
\hline$\omega_{t}$ & Transmitted shock wave angle & Equation (4.1.2) \\
\hline
\end{tabular}




\section{Chapter 1}

\section{INTRODUCTION}

When a plane shock wave propagates through a non-uniform medium, the wave front becomes curved and distorted, as in the diffraction and refraction of shock waves in turbulence and in substances of varying sound speed. Similar distortions of shock fronts occur even in uniform media when the fronts pass over curved boundaries, as in shock diffraction over a wedge. The latter problem has received considerable attention in the past, and it is now possible to treat diffraction over bodies with exact numerical calculation (Shankar, Kutler and Anderson, 1978; Kutler and Shankar, 1977), or with an approximate theory, known as shock dynamics, due to Whitham (1957, 1959). This approximate theory has been extended by Collins and Chen (1970b), to cover shock propagation in a non-uniform medium. However, their work has not received much attention, despite the fact that there does not exist a method for calculating such flows that properly preserves the sharpness of discontinuities, other than the method of characteristics for fully 2 - or 3-dimensional non-steady flow.

Shock dynamics is the nonlinear analogue of geometrical acoustics. It accounts for the fact that in non-uniform propagation, the rays (normals to the shock front) are not straight and parallel. It treats the distortion of wave fronts in terms of disturbances which propagate transversely along the fronts. As with geometrical acoustics, the theory does not treat the field behind the wave fronts and, therefore, is not applicable to problems in which disturbances generated by processes behind the shock overtake and modify it. It differs from geometrical acoustics in that the disturbances may either steepen or spread out by nonlinearity, a property unique to finite-amplitude waves. 
It is the purpose of this work to reexamine the theory of shock dynamics for application to the problem of shock propagation in a non-uniform medium, and to study the behaviour in a few simple cases. In particular, we consider the case of a plane shock wave propagating at some angle to a free plane gaseous interface separating two uniform regions with different sound speeds. The general results of such an interaction are the refraction of the wave as it crosses the interface, and the generation of a reflected wave. The reflected wave may be either a shock or an expansion, depending on the gas properties on each side of the interface, and on the local angle of incidence. Two basic types of shock refraction have been observed in experiments (Jahn, 1956; Abd-el-Fattah, Henderson and Lozzi, 1976). The first is regular refraction, which is specular in nature, and involves either a shock or an expansion for the reflected wave, while the second is irregular or Mach refraction, in which the reflected wave can only be a shock. Irregular refraction involves the formation of a third shock at the interface, called the Mach stem, and there exists a vortex sheet discontinuity downstream of the three shock confluence. In general, regular refraction occurs when the angle between the shock front and the interface is small, while irregular refraction occurs when the angle of incidence is large.

Although the governing equations for the regular refraction of a plane shock at a free plane gaseous interface are relatively simple to formulate, their analysis is difficult because of the number of parameters involved (Taub, 1947; Polachek and Seeger, 1951; Henderson, 1966 and others). In general, the equations have twelve possible roots. Some of the roots may be imaginary or may yield thermodynamically impossible solutions, and these roots can be eliminated. However, it is still necessary to develop a criterion to determine which of the remaining solutions agrees best with experiment. This has been the source of considerable controversy, and in view of this complexity, a method of analyzing the shock refraction problem by a simpler approximate theory is attractive. 
In addition, a theory that can handle irregular refraction, as well as arbitrarily complex geometrical boundary conditions, will be very useful.

Whitham $(1957,1959)$ proposed an approximate theory for the propagation of a shock in a perfect gas, which he termed shock dynamics. The theory involves the treatment of wave-like disturbances, which propagate on the shock front and carry information on the changes of shock strength and ray angle. The governing equations for these disturbances are hyperbolic in nature, and their behaviour is analogous in many ways to that of compression and expansion waves in gasdynamics. In particular, a disturbance carrying an increase in shock strength steepens and eventually breaks to form a discontinuity, while a disturbance carrying a decrease in shock strength spreads out along the shock front.

The shock may be thought of as propagating along channels made up by neighbouring rays on its front. Immediately behind the shock front, the rays are in fact the particle paths, so we may think of the shock as locally travelling down a channel with solid walls. Chester (1954) and Chisnell (1957) have shown that a first-order differential relationship can be derived between the shock strength and the changes in channel area, if the rate of change of channel area is small. This relationship plays an important role in the overall development of the theory.

One restriction to the application of shock dynamics for the study of general shock propagation problems is that no disturbances generated in the flow behind the shock should overtake and interact with the main shock front, so that in effect, the flow field behind the wave front is ignored and cannot be calculated. The reason for this stems from the application of Whitham's characteristic rule. 
Although the theory of shock dynamies is approximate, it has been developed and applied successfully to various problems of shock propagation in a uniform medium. In particular, it has been used to solve problems involving the diffraction of strong shocks by cones, cylinders and spheres (Bryson and Gross, 1961), and the diffraction of shocks by wedges and corners (Whitham, 1957; Henderson, 1980). In general, for Mach numbers greater than about 2.0, the agreement between the theoretical and the experimental results is found to be good.

Collins and Chen (1970b) have applied the theory of shock dynamics to the propagation of a shock wave through a non-uniform medium. They have attempted to solve the problem of a shock propagating in two half-planes separated by a free surface, by imposing the condition that the two portions of the shock front at the free surface travel at the same velocity. While the concept is sound, the analysis does not properly account for the transmission and reflection of both families of characteristics at the interface, and does not provide for the formation of discontinuities when disturbances of increasing strength propagate on the shock.

The present work reformulates the theory of shock dynamics in a nonuniform medium, using Whitham's characteristic rule, and obtains a completely general expression for the area-Mach number relationship that accounts for any spatial variations in the medium ahead of the shock front. As mentioned above, a case of special interest is that in which discontinuities in fluid properties, that is, contact discontinuities, occur ahead of the shock front. We develop a method for treating these discontinuities, and show that the theory leads naturally to an expression analogous to Snell's law of refraction (the same condition imposed by Collins and Chen), together with an ordinary differential equation that relates the Mach numbers on both sides of the discontinuity. These two 
relations are sufficient for closure of the system of equations.

In Chapter 2 , the equations of shock dynamics are reformulated to include the effects of changes of sound speed in the medium ahead of the shock front, which may be caused by spatial variations in thermodynamic state or gas concentration, or by the presence of body forces. The resulting equations are nonlinear and are similar to the conventional shock dynamies equations, except for the appearance of source terms. It is these source terms which generate disturbances on the shock front as the shock propagates through the nonuniformities. In Chapter 3 , the equations are applied to two different problems involving a plane shock wave incident at some angle to a free plane gaseous interface. In the first problem, only a gaseous interface is present, while the second involves a solid boundary as well. Both problems were chosen because they are self-similar and do not contain any non-simple regions. In Chapter 4, a comparison of results is made with the experimental data of Jahn (1956) and with the experimental and theoretical work of Henderson's group (Abd-elFattah, Henderson and Lozzi, 1976; Abd-el-Fattah and Henderson, 1978). Finally, in the summary and conclusions, some points for further investigation are set out. 


\section{Chapter 2}

\section{THE GOVERNING EQUATIONS}

The derivation of the equations which account for the generation and propagation of disturbances on the shock front follows the analysis of Whitham (1957). Starting with the equations of motion, in section 2.1, we form a differential relation for the flow quantities on the $C^{+}$characteristic, apply Whitham's characteristic rule, and use the shock jump conditions to obtain a relationship between the shock Mach number, the ray tube area and the local changes in the sound speed of the medium ahead of the shock wave. Next, in section 2.2, we introduce an orthogonal coordinate system based on the shock positions and the rays, and from purely geometrical considerations, formulate two further equations. In section 2.3, the three equations are combined, put into characteristic form and transformed into the physical coordinate system, to. yield the governing equations for the theory. Finally, in section 2.4, we discuss the behaviour of the characteristics, both in uniform and non-uniform regions, and derive the jump conditions at discontinuities in the flow.

\subsection{Quasi-one-dimensional non-steady fow in a non-uniform medium with finite area changes}

In this section, a relation between the shock Mach number $M$, the ray tube area $A$ and variation in fluid properties is obtained as in CCW theory (Chester, 1954; Chisnell, 1955, 1957; Whitham, 1958) for shock propagation in a channel of slowly varying area, and, in this case, with slowly varying sound speed $a_{0}$ ahead of the shock. Since shock dynamics is formulated to treat only perfect gases, the changes in $a_{0}$ may be caused by spatial variations in the thermodynamic state of the fluid, or by spatial variations in the concentration of mixtures of 
perfect gases, or both. Hence, in the formulation of the theory, it is necessary to consider imposed variations of temperature $T_{0}$ and specific heat ratio $\gamma$, together with related variations in density $\rho_{0}$, and of pressure $p_{0}$, which would result if body forces were present. It is convenient to express these variations in terms of $\gamma, a_{0}$ and $p_{0}$.

We consider the propagation of a shock wave down a tube of non-uniform cross-section which contains an equilibrium non-homogeneous distribution of gas. If the cross-sectional area $A(x)$ of the tube does not vary too rapidly, that is,

$$
\frac{A(x)-A}{A} \ll 1
$$

where $A$ is some mean value, then the fow may be treated as quasi-one dimensional, and the equations obtained by averaging across the cross-section may be applied. The equations of motion are

continuity :

$$
A \frac{\partial \rho}{\partial t}+\frac{\partial}{\partial x}(\rho u A)=0
$$

momentum :

$$
\frac{D u}{D t}+\frac{1}{\rho} \frac{\partial p}{\partial x}=F
$$

and energy:

$$
\frac{D p}{D t}-a^{2} \frac{\partial \rho}{\partial x}=0
$$

where $F$ is the body force per unit mass. These equations can be rewritten as 


$$
\begin{array}{r}
\rho_{t}+\rho u_{x}+u \rho_{x}+\rho u \frac{A_{x}}{A}=0, \\
u_{t}+u u_{z}+\frac{1}{\rho} p_{z}=F
\end{array}
$$

and

$$
p_{t}+u p_{x}-\alpha^{2}\left(\rho_{t}+u p_{x}\right)=0
$$

Consider the flow quantities $\alpha_{1}, p_{1}, u_{1}$ and $\rho_{1}$ behind the shock. We eliminate the derivatives of $\rho$ from (2.1.5) and (2.1.6) to give

$$
p_{t}+u_{1} p_{x}+p_{1} \alpha_{1}^{2}\left(u_{x}+u_{1} \frac{A_{x}}{A}\right)=0
$$

and rearrange $(2.1 .6)$ to give

$$
p_{x}+\rho_{1} u_{t}+\rho_{1} u_{1} u_{z}=\rho_{1} F
$$

Multiply (2.1.9) by $l$ and add to (2.1.8) to give

$$
p_{t}+u_{1} p_{x}+\rho_{1} \alpha_{1}^{2}\left(u_{x}+u_{1} \frac{A_{x}}{A}\right)+l\left(p_{z}+\rho_{1} u_{t}+\rho_{1} u_{1} u_{x}-\rho_{1} F\right)=0
$$

that is,

$$
\left\{p_{t}+\left(u_{1}+l\right) p_{x}\right\}+\rho_{1} l\left\{u_{t}+\frac{1}{l}\left(a_{1}^{2}+u_{1} l\right) u_{x}\right\}+\rho_{1} a_{1}^{2} u \frac{A_{x}}{A}-\rho_{1} l F=0 .
$$

To put this into characteristic form, we require 


$$
\left(u_{1}+l\right)=\frac{1}{l}\left(a_{1}^{2}+u_{1} l\right)
$$

that is,

$$
l= \pm a_{1} .
$$

So, the slopes of the two characteristic curves given by

$$
\frac{d x}{d t}=u_{1}+l
$$

are

$$
\frac{d x}{d t}=u_{1} \pm a_{1}
$$

and the characteristic equations are

$$
C^{ \pm}:\left\{\frac{\partial}{\partial t}+\left(u_{1} \pm a_{1}\right) \frac{\partial}{\partial x}\right\}\left(p \pm \rho_{1} \alpha_{1} u\right)+\rho_{1} a_{1}^{2} u_{1} \frac{A_{x}}{A} \mp \rho_{1} a_{1} F=0 \text {. }
$$

Also, from (2.1.7) we get a third characteristic equation

$$
P^{+}: \quad\left\{\frac{\partial}{\partial t}+u_{1} \frac{\partial}{\partial x}\right\}\left(p-a_{1}^{2} \rho\right)=0 .
$$

Next, we apply Whitham's characteristic rule, in which the exact nonlinear differential relation for the flow quantities along the $C^{+}$characteristic is used for the flow quantities just behind the shock front. The characteristic rule is discussed by Whitham (1958), who proves its direct applicablity for the case of small perturbations in the ray tube area. In the nonlinear case, that is, when changes in the ray tube area are not necessarily small, the rule still works remarkably well for "self-propagating" shocks, that is, for shocks which are 
accelerating, so that disturbances located farther than a certain critical distance behind the shock never catch up with the shock (Hayes, 1968). It is implicitly assumed in the application of the characteristic rule that this condition is satisfied, that is, we exclude the possibility of any disturbances from the downstream flow overtaking and modifying the shock.

From (2.1.10), on the $C^{+}$characteristic, we have

$$
\frac{d}{d x}\left(p+\rho_{1} a_{1} u\right)+\frac{\rho_{1} a_{1}^{2} u_{1}}{u_{1}+a_{1}} \frac{1}{A} \frac{d A}{d x}-\frac{\rho_{1} a_{1} F}{u_{1}+a_{1}}=0 \text { on } \frac{d x}{d t}=u_{1}+a_{1}
$$

By application of the characteristic rule, on the shock front, we have

$$
\frac{d p_{1}}{d x}+\rho_{1} \alpha_{1} \frac{d u_{1}}{d x}+\frac{\rho_{1} \alpha_{1}^{2} u_{1}}{u_{1}+\alpha_{1}} \frac{1}{A} \frac{d A}{d x}-\frac{\rho_{1} \alpha_{1} F}{u_{1}+\alpha_{1}}=0
$$

which we rearrange to give

$$
\frac{u_{1}+a_{1}}{\rho_{1} \bar{u}_{1}^{2} u_{1}} d p_{1}+\frac{u_{1}+a_{1}}{a_{1} u_{1}} d u_{1}+\frac{1}{A} d A-\frac{1}{a_{1} u_{1}} F d x=0
$$

Using the sound speed relation for a perfect gas

$$
a_{0}^{2}=\gamma \frac{\rho_{0}}{\rho_{0}}
$$

and the equilibrium condition, that is,

$$
F=\frac{1}{\rho_{0}} \frac{d p_{0}}{d x}
$$


we can write (2.1.14) as

$$
P \frac{d p_{1}}{p_{0}}+Q \frac{d u_{1}}{u_{1}}-R \frac{d p_{0}}{p_{0}}+\frac{d A}{A}=0
$$

where

$$
\begin{aligned}
& P=\frac{1}{\gamma}\left[\frac{\frac{u_{1}}{a_{0}}+\frac{a_{1}}{a_{0}}}{\frac{\rho_{1}}{\rho_{0}}\left(\frac{a_{1}}{a_{0}}\right)^{2} \frac{u_{1}}{a_{0}}}\right] \\
& Q=\left[\frac{\frac{u_{1}}{a_{0}}+\frac{a_{1}}{a_{0}}}{\frac{a_{1}}{a_{0}}}\right]
\end{aligned}
$$

and

$$
R=\frac{1}{\gamma}\left[\frac{1}{\frac{a_{1}}{a_{0}} \frac{u_{1}}{a_{0}}}\right] .
$$

We now use the shock jump conditions to relate the perturbation quantities $d p_{1}$ and $d u_{1}$ to the changes in Mach number. We have the shock relations

$$
\begin{aligned}
& u_{1}=\frac{2 a_{0}\left(M^{2}-1\right)}{(\gamma+1) M} \\
& p_{1}=p_{0}\left[1+\frac{2 \gamma\left(M^{2}-1\right)}{\gamma+1}\right],
\end{aligned}
$$

where $M$ is the local shock Mach number, and we have assumed that $u_{0}=0$. Differentiation of (2.1.16) yields 


$$
\frac{d u_{1}}{u_{1}}=\frac{d a_{0}}{a_{0}}-\frac{d \gamma}{\gamma+1}+\frac{M^{2}+1}{M\left(M^{2}-1\right)} d M
$$

and

$$
d p_{1}=\frac{2 p_{0}}{\gamma+1}\left[2 \gamma M d M+\frac{M^{2}-1}{\gamma+1} d \gamma\right]+\left[1+\frac{2 \gamma\left(M^{2}-1\right)}{\gamma+1}\right] d p_{0}
$$

We substitute from $(2.1 .17)$ and $(2.1 .18)$ into $(2.1 .15)$ to give

$$
\begin{aligned}
& \left(\frac{2 P}{\gamma+1}\right)\left[2 \gamma M d M+\frac{M^{2}-1}{\gamma+1} d \gamma\right]+P\left[1+\frac{2 \gamma\left(M^{2}-1\right)}{\gamma+1}\right] \frac{d p_{0}}{p_{0}}+ \\
& +Q\left[\frac{d \alpha_{0}}{\alpha_{0}}-\frac{d \gamma}{\gamma+1}+\frac{M^{2}+1}{M\left(M^{2}-1\right)} d M\right]-R \frac{d p_{0}}{p_{0}}+\frac{d A}{A}=0 .
\end{aligned}
$$

which can be rewritten as

$$
\frac{M \lambda}{M^{2}-1} d M+\frac{d A}{A}+f d \gamma+g \frac{d a_{0}}{a_{0}}+h \frac{d p_{0}}{p_{0}}=0
$$

where

$$
\begin{gathered}
\lambda(M, \gamma)=\left(1+\frac{2}{\gamma+1} \frac{1-\mu^{2}}{\mu}\right)\left(1+2 \mu+\frac{1}{M^{2}}\right), \\
f(M, \gamma)=\frac{g(M, \gamma)}{\gamma(\gamma+1)}(\mu-\gamma),
\end{gathered}
$$




$$
\begin{gathered}
g(M, \gamma)=1+\frac{2 \mu\left(M^{2}-1\right)}{(\gamma-1) M^{2}+2} \\
h(M, \gamma)=\frac{1}{2 \gamma\left(M^{2}-1\right)}\left[2\left(M^{2}-1\right)+\mu\left\{2 \gamma M^{2}-(\gamma-1)\right\}-\frac{(\gamma+1)^{2} \mu M^{2}}{(\gamma-1) M^{2}+2}\right]
\end{gathered}
$$

and

$$
\mu(M, \gamma)=\left[\frac{(\gamma-1) M^{2}+2}{2 \gamma M^{2}-(\gamma-1)}\right]^{1 / 2}
$$

We note that equation (2.1.20) treats any spatial variations in the perfect gas ahead of the shock front. In general, it agrees with the expression derived by Collins and Chen (1970b), except for the coefficients of $d^{d} \gamma$ and $d p_{0}$. In the case of the latter coefficient, the difference is due to the fact that in their analysis, Collins and Chen do not consider the possible occurrence of body forces.

Equation (2.1.20) corresponds to Whitham's area-Mach number relation for shock propagation in a uniform medium, to which it reduces immediately when $\alpha_{0}, p_{0}$ and $\gamma$ are constant. Then we have

$$
\frac{d A}{A}+\frac{M \lambda(M)}{M^{2}-1} d M=0
$$

Thus, in a uniform medium, $A=A(M)$ only, whereas in a non-uniform medium, $A=A\left(M, \alpha_{0}, p_{0}, \gamma\right)$. Details of the integration of (2.1.26) are given in Appendix A. 


\subsection{Geometrical shock dynamics in two dimensions}

An orthogonal system of coordinates $(\alpha, \beta)$, is introduced, where $\alpha=$ constant are the shock fronts and $\beta=$ constant are the rays. Following whitham (1959), we specify the shock surface as

$$
S(\mathrm{x}, t) \equiv t-\alpha(\mathrm{x})=0
$$

that is, the family of lines $\alpha(x)=$ constant describes the positions of the shock front at successive times. An increment $\delta \alpha$ corresponds to a change of shock position in time $\delta t$, where

$$
\delta t=\delta \alpha
$$

If $U(\alpha, \beta)$ is the normal velocity of the shock front at $(\alpha, \beta)$, the distance travelled by the shock in time ot is

$$
U(\alpha, \beta) \delta t=U(\alpha, \beta) \delta \alpha
$$

Thus, the distance along the ray between the shock positions given by $\alpha$ and $\alpha+\delta \alpha$ is

$$
\mathrm{PQ}=U(\alpha, \beta) \delta \alpha
$$

where $\mathrm{P}$ is the point with the coordinates $(\alpha, \beta)$ as shown in figure 2.1. In terms of the local Mach number, defined by

$$
M(\alpha, \beta)=\frac{U(\alpha, \beta)}{a_{0}(\alpha, \beta)}
$$

we can write 


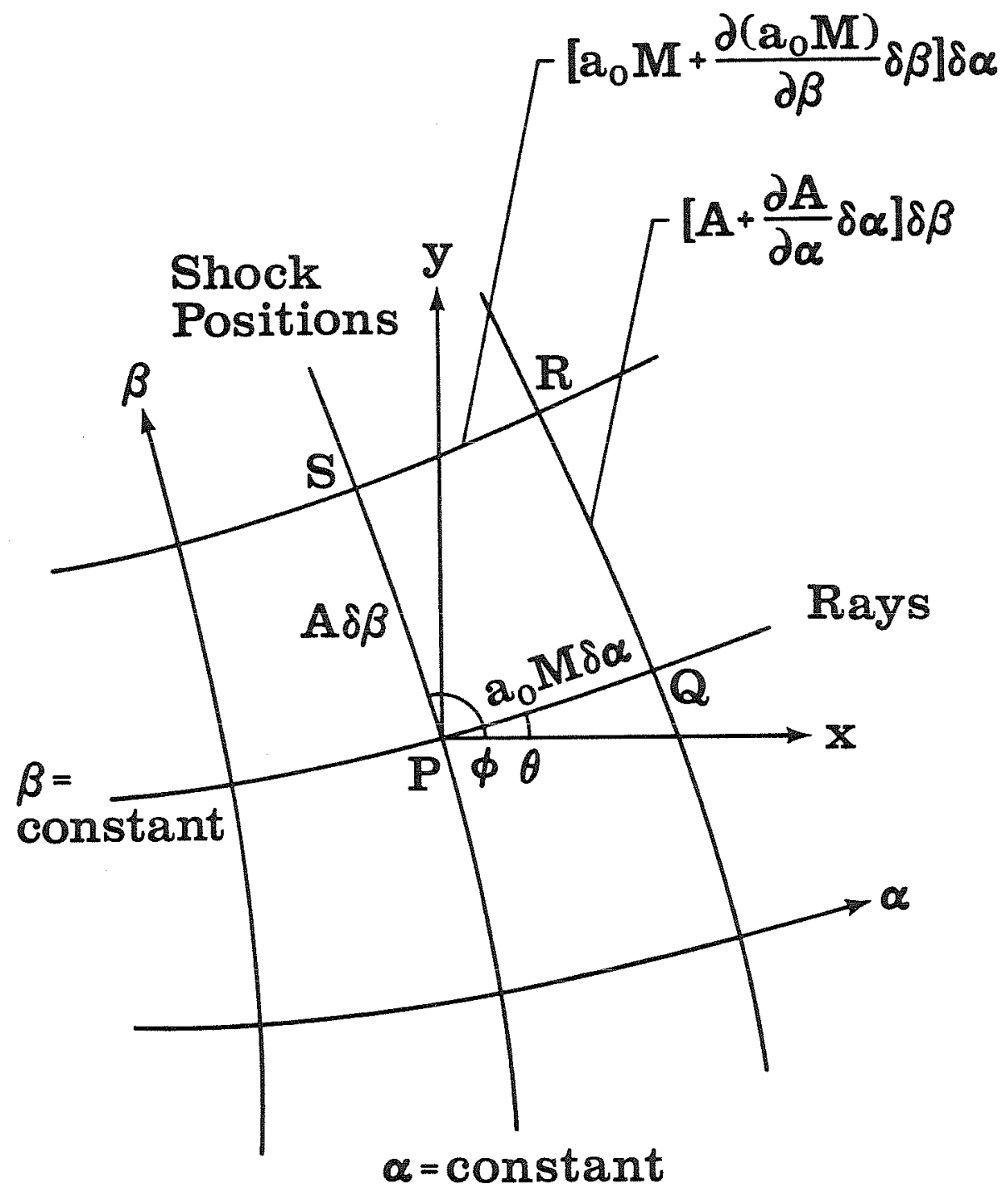

FIGURE 2.1. The $(\alpha, \beta)$ coordinate system. 


$$
P Q=a_{0}(\alpha, \beta) M(\alpha, \beta) \delta \alpha
$$

Next, we consider increments in the $\beta$-direction. We let the distance along the shock between the rays described by $\beta$ and $\beta+\delta \beta$ be

$$
\mathrm{PS}=A(\alpha, \beta) \delta \beta
$$

where $A(\alpha, \beta)$ is the area of the ray tube bounded by the rays $\beta$ and $\beta+\delta \beta$. From the geometry of figure 2.1, we have

$$
\mathrm{SR}=\left[\boldsymbol{\alpha}_{0} M+\frac{\partial}{\partial \beta}\left(\boldsymbol{\alpha}_{0} M\right) \delta \beta\right] \delta \alpha .
$$

and

$$
\mathrm{QR}=\left[A+\frac{\partial A}{\partial \alpha} \delta \beta\right] \delta \alpha
$$

Let $\phi(\alpha, \beta)$ be the angle between the shock and the $x$-axis. Then the change in shock inclination from $P$ to $Q$ is

$$
\begin{aligned}
\delta \phi & =-\frac{\mathrm{SR}-\mathrm{PQ}}{\mathrm{PS}} \\
& =-\frac{\frac{\partial}{\partial \beta}\left(a_{0} M\right) \delta \beta \delta \alpha}{A \delta \beta} .
\end{aligned}
$$

that is,

$$
\frac{\delta \phi}{\delta \alpha}=-\frac{1}{A} \frac{\partial}{\partial \beta}\left(a_{0} M\right)
$$

Similarly, let $\theta(\alpha, \beta)$ be the angle between the ray and the $x$-axis. Then the change in ray inclination from $P$ to $S$ is 


$$
\begin{aligned}
\delta \theta & =\frac{\mathrm{QR}-\mathrm{PS}}{\mathrm{PQ}} \\
& =-\frac{\frac{\partial A}{\partial \alpha} \delta \alpha \delta \beta}{\alpha_{0} M \delta \alpha}
\end{aligned}
$$

that is,

$$
\frac{\delta \theta}{\delta \beta}=\frac{1}{a_{0} M} \frac{\partial A}{\partial \alpha}
$$

From the orthogonality condition, we have

$$
\phi=\frac{\pi}{2}+\theta
$$

Applying (2.2.7) and passing to the limit, (2.2.5) and (2.2.6) become

$$
\frac{\partial \theta}{\partial \alpha}=-\frac{1}{A} \frac{\partial}{\partial \beta}\left(\alpha_{0} M\right)
$$

and

$$
\frac{\partial \theta}{\partial \beta}=\frac{1}{a_{0} M} \frac{\partial A}{\partial \alpha}
$$

Equations (2.2.8) relate the quantities $a_{0}, A, M$ and $\theta$ in the $(\alpha, \beta)$ plane.

\subsection{Wave propagation on the shock}

We now combine equation (2.1.20) with equations (2.2.8) to obtain a system of equations which decribes the propagation of a disturbance on the shock front. From (2.1.20), we have

$$
\frac{\partial A}{\partial \alpha}=-A\left[\frac{M \lambda}{M^{2}-1} \frac{\partial M}{\partial \alpha}+f \frac{\partial y}{\partial \alpha}+\frac{g}{\alpha_{0}} \frac{\partial \alpha_{0}}{\partial \alpha}+\frac{h}{p_{0}} \frac{\partial p_{0}}{\partial \alpha}\right]
$$


On substituting (2.3.1) into (2.2.B), we get

$$
\frac{\partial \theta}{\partial \alpha}+\frac{1}{A} \frac{\partial}{\partial \beta}\left(a_{0} M\right)=0
$$

and

$$
\frac{\partial \theta}{\partial \beta}+\frac{A}{a_{0} M}\left[\frac{M \lambda}{M^{2}-1} \frac{\partial M}{\partial \alpha}+f \frac{\partial \gamma}{\partial \alpha}+\frac{g}{a_{0}} \frac{\partial a_{0}}{\partial \alpha}+\frac{h}{p_{0}} \frac{\partial p_{0}}{\partial \alpha}\right]=0 .
$$

Equations (2.3.2) may be rewritten as

$$
\frac{\partial \theta}{\partial \alpha}+\frac{a_{0}}{A} \frac{\partial M}{\partial \beta}+\frac{M}{A} \frac{\partial a_{0}}{\partial \beta}=0
$$

and

$$
\frac{\partial \theta}{\partial \beta}+\frac{A \lambda}{\alpha_{0}\left(M^{2}-1\right)} \frac{\partial M}{\partial \alpha}+\frac{A}{\alpha_{0} M}\left[f \frac{\partial \gamma}{\partial \alpha}+\frac{g}{a_{0}} \frac{\partial \alpha_{0}}{\partial \alpha}+\frac{\hbar}{p_{0}} \frac{\partial p_{0}}{\partial \alpha}\right]=0 \text {. }
$$

We introduce the simplifying notation

$$
\begin{aligned}
& \xi=\frac{a_{0}}{A}, \\
& \kappa=\frac{A \lambda}{a_{0}\left(M^{2}-1\right)}, \\
& F=\frac{M}{A} \frac{\partial a_{0}}{\partial \beta}
\end{aligned}
$$

and

$$
G=\frac{A}{a_{0} M}\left[f \frac{\partial \gamma}{\partial \alpha}+\frac{g}{a_{0}} \frac{\partial a_{0}}{\partial \alpha}+\frac{h}{p_{0}} \frac{\partial p_{0}}{\partial \alpha}\right]
$$

so that equations (2.3.3) become 


$$
\frac{\partial \theta}{\partial \alpha}+\xi \frac{\partial M}{\partial \beta}+F=0
$$

and

$$
\frac{\partial \theta}{\partial \beta}+\kappa \frac{\partial M}{\partial \alpha}+G=0
$$

We multiply (2.3.7) by $l$ and add to (2.3.6) to give

$$
\left(\theta_{\alpha}+\xi M_{\beta}+F\right)+l\left(\theta_{\beta}+\kappa M_{\alpha}+G\right)=0 .
$$

that is,

$$
\left(\theta_{\alpha}+l \theta_{\beta}\right)+l \kappa\left(M_{\alpha}+\frac{\xi}{l \kappa} M_{\beta}\right)+(F+l G)=0 .
$$

To put this equation into characteristic form, we require

$$
l=\frac{\xi}{l \kappa} \text {, }
$$

that is,

$$
l=\left(\frac{\xi}{\kappa}\right)^{1 / 2} .
$$

So, the slopes of the two characteristic curves given by

$$
\frac{d \beta}{d \alpha}=l
$$

are

$$
\frac{d \beta}{d \alpha}= \pm \frac{\alpha_{0}}{A}\left(\frac{M^{2}-1}{\lambda}\right)^{1 / 2}
$$

and the characteristic equations $C^{ \pm}$are 


$$
\left(\theta_{a} \pm c \theta_{\beta}\right) \pm c \kappa\left(M_{\alpha} \pm c M_{\beta}\right)+(F \pm c G)=0
$$

that is,

$$
\left(\theta_{\alpha} \pm c \theta_{\beta}\right) \pm\left(\frac{\lambda}{M^{2}-1}\right)^{1 / 2}\left(M_{\alpha} \pm c M_{\beta}\right)=-(F \pm c G)
$$

where

$$
c=\frac{a_{0}}{A}\left(\frac{M^{2}-1}{\lambda}\right)^{1 / 2}
$$

From (2.3.8), we get

$$
d \theta \pm\left(\frac{\lambda}{M^{2}-1}\right)^{1 / 2} d M=-(F \pm c G) d \alpha \text { on } \quad \frac{d \beta}{d \alpha}= \pm c
$$

If we introduce

$$
d \omega=\left(\frac{\lambda}{M^{2}-1}\right)^{1 / 2} d M
$$

that is,

$$
\omega=\int_{1}^{H}\left[\frac{\lambda(m, \gamma)}{m^{2}-1}\right]^{1 / 2} d m
$$

the characteristic equations (2.3.8) become 


$$
C^{ \pm}: \quad\left(\frac{\partial}{\partial \alpha} \pm c \frac{\partial}{\partial \beta}\right)(\theta \pm \omega)=-(F \pm c G)
$$

while equations (2.3.10) become

$$
d \theta \pm d \omega=-(F \pm c G) d \alpha
$$

on the characteristics

$$
\frac{d \beta}{d \alpha}= \pm c
$$

Since, in general, problems are more easily formulated in the $(x, y)$ plane than in the $(\alpha, \beta)$ plane, it is expedient to transform the governing equations to the physical plane. The coordinate transformation relations, which are derived in Appendix B, are applied to (2.3.12) to give

$$
\begin{aligned}
& {\left[\left(a_{0} M \cos \theta \frac{\partial}{\partial x}+a_{0} M \sin \theta \frac{\partial}{\partial y}\right) \pm c\left(-A \sin \theta \frac{\partial}{\partial x}+A \cos \theta \frac{\partial}{\partial y}\right)\right](\theta \pm \omega)=} \\
& =\int \frac{M}{A}\left(-A \sin \theta \frac{\partial a_{0}}{\partial x}+A \cos \theta \frac{\partial \alpha_{0}}{\partial y}\right) \pm \frac{A c}{\alpha_{0} M}\left\{f\left(\alpha_{0} M \cos \theta \frac{\partial \gamma}{\partial x}+\alpha_{0} M \sin \theta \frac{\partial y}{\partial y}\right)+\right. \\
& \left.\left.+\frac{g}{a_{0}}\left(a_{0} M \cos \theta \frac{\partial a_{0}}{\partial x}+a_{0} M \sin \theta \frac{\partial a_{0}}{\partial y}\right)+\frac{h}{p_{0}}\left(a_{0} M \cos \theta \frac{\partial p_{0}}{\partial x}+a_{0} M \sin \theta \frac{\partial p_{0}}{\partial y}\right)\right]\right\} .
\end{aligned}
$$

On rearranging, this becomes 


$$
\begin{aligned}
& {\left[(1 \mp \nu \tan \theta) \frac{\partial}{\partial x}+(\tan \theta \pm \nu) \frac{\partial}{\partial y}\right](\theta \pm \omega)=} \\
& =-\left[\frac{1}{a_{0}}\{-\tan \theta \pm \nu g) \frac{\partial a_{0}}{\partial x}+(1 \pm \nu g \tan \theta) \frac{\partial a_{0}}{\partial y}\right\}+ \\
& \left.\quad \pm \nu f\left(\frac{\partial \gamma}{\partial x}+\tan \theta \frac{\partial \gamma}{\partial y}\right) \pm \frac{\nu h}{p_{0}}\left(\frac{\partial p_{0}}{\partial x}+\tan \theta \frac{\partial p_{0}}{\partial y}\right)\right]
\end{aligned}
$$

where

$$
\nu=\frac{A \mathrm{c}}{a_{0} M} .
$$

that is,

$$
\nu=\frac{1}{M}\left(\frac{M^{2}-1}{\lambda}\right)^{1 / 2}
$$

Similarly, equations (2.3.13) become

$$
\begin{aligned}
d \theta \pm d \omega= & -\frac{1}{1 \mp \nu \tan \theta}\left[\frac{1}{\alpha_{0}}\left\{(-\tan \theta \pm \nu g) \frac{\partial \alpha_{0}}{\partial x}+(1 \pm \nu g \tan \theta) \frac{\partial \alpha_{0}}{\partial y}\right\}+\right. \\
& \left. \pm \nu f\left(\frac{\partial \gamma}{\partial x}+\tan \theta \frac{\partial \gamma}{\partial y}\right) \pm \frac{\nu h}{p_{0}}\left(\frac{\partial p_{0}}{\partial x}+\tan \theta \frac{\partial p_{0}}{\partial y}\right)\right] d x
\end{aligned}
$$

and 


$$
\begin{aligned}
d \theta \pm d \omega= & -\frac{1}{\tan \theta \pm \nu}\left[\frac{1}{a_{0}}\left\{(-\tan \theta \pm \nu g) \frac{\partial a_{0}}{\partial x}+(1 \pm \nu g \tan \theta) \frac{\partial a_{0}}{\partial y}\right\}+\right. \\
& \left. \pm \nu f\left(\frac{\partial \gamma}{\partial x}+\tan \theta \frac{\partial y}{\partial y}\right) \pm \frac{\nu \hbar}{p_{0}}\left(\frac{\partial p_{0}}{\partial x}+\tan \theta \frac{\partial p_{0}}{\partial y}\right)\right] d y
\end{aligned}
$$

on the characteristics

$$
\begin{aligned}
\frac{d y}{d x} & =\frac{\tan \theta \pm \nu}{1 \mp \nu \tan \theta} \\
& =\tan (\theta \pm v)
\end{aligned}
$$

where

$$
v=\tan ^{-1}(\nu)
$$

The angles made by the $C^{+}$and $C^{-}$characteristics with the $x$-axis are given by

$$
\eta^{ \pm}=\theta \pm v
$$

We note that the two characteristics make equal angles with the ray direction. The behaviour of each of the functions $A, \lambda, \mu, \nu, v$ and $\omega$ with $M$ is shown in figure 2.2.

Equations (2.3.14), or alternatively, equations (2.3.16) and (2.3.17), form the governing equations for shock dynamies in a non-uniform medium. 


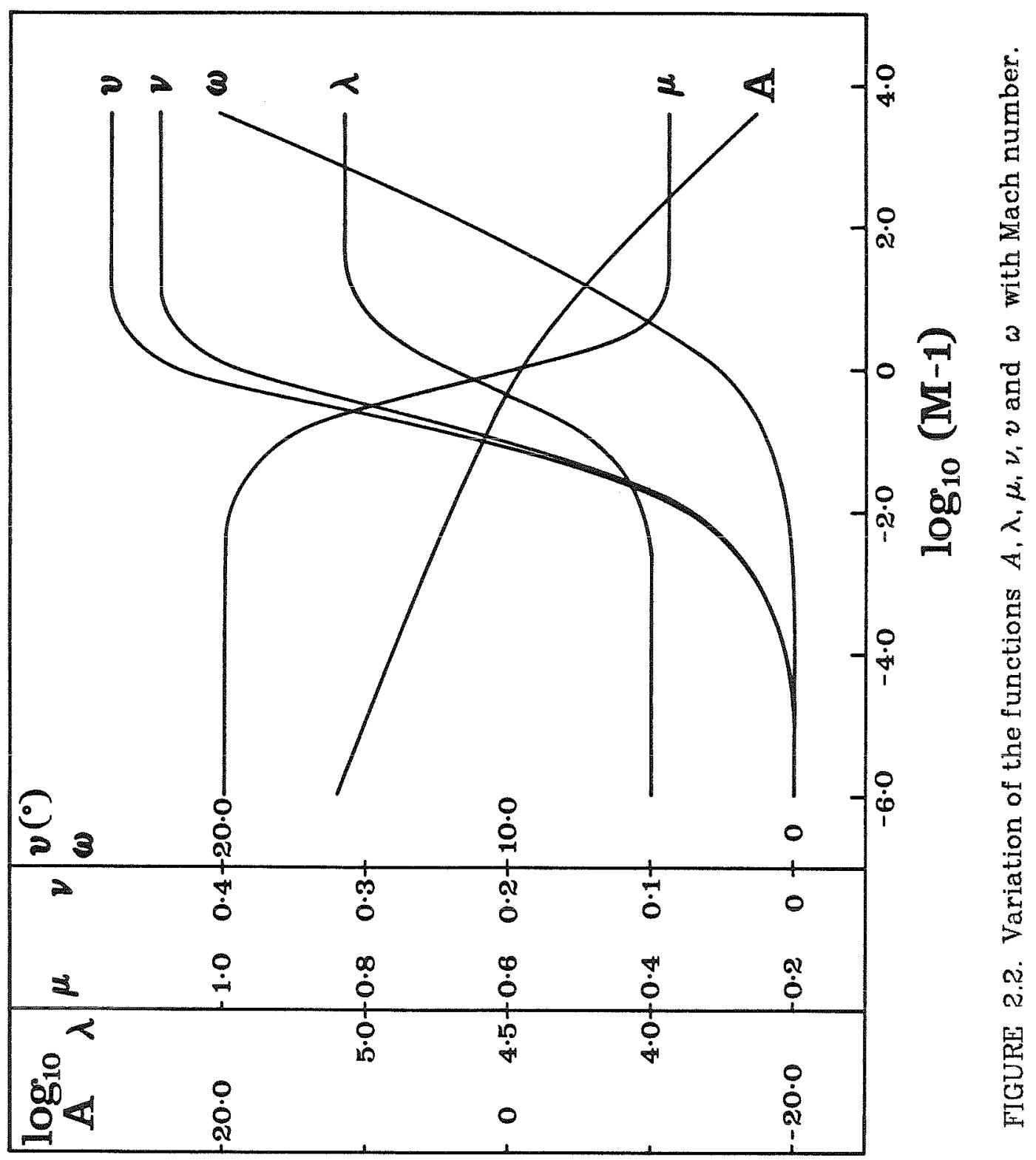




\subsection{The characteristic system}

The governing equations (2.3.14) form a hyperbolic system which describes wave-like disturbances that travel in opposite directions along the shock front, and carry information about changes of $M$ and $\theta$. The characteristics are real, and their slope, $d y / d x$, is an increasing function of $M$, so that a disturbance carrying an increase in shock strength steepens, whereas a disturbance carrying a decrease in shock strength spreads out. When an expansive disturbance on the shock front results in a simple wave (expansion fan), we refer to it as a shock-expansion. On the other hand, a compressive disturbance eventually breaks and forms a discontinuity of $M$ and $\theta$ on the shock front, the jump conditions across which have been given by Whitham (1957). The locus of this discontinuity is called a shock-shock. Physically, the shock-shock is the locus of the triple-shock intersection, and its occurrence signals the formation of a Mach stem.

The governing equations (2.3.14) are similar to those obtained by Whitham (1957) for the case of a uniform medium $\left(\gamma, \alpha_{0}, p_{0}=\right.$ constant), but in general, they differ through the appearance of "source" terms on the right-hand side. The source terms contain the gradients of the independent variables, $\gamma, a_{0}$ and $p_{0}$. Indeed, for the uniform medium case, the gradients are zero and the source terms vanish, so that we recover the equations given by Whitham. Thus, in this formulation, disturbances may be generated on the shock front by boundary conditions such as wall curvature, as well as by non-uniformities in the medium ahead of the shock. We note that the source terms are implicit, since they contain the dependent variables, $M$ and $\theta$, as well as the independent variables, $\gamma_{1} a_{0}$ and $p_{0}$ 
2.4.1. The shock-expansion and the shock-shock. The governing equations may be solved by the method of characteristics for two independent variables. In the uniform regions, we have

$$
\theta \pm \omega=J^{ \pm}
$$

where $J^{ \pm}$are invariants. In a shock-expansion, the invariants are constant on the family of characteristics which originate in the uniform region, while the other family of characteristics carries the disturbance. On the other hand, when shock-shocks occur, they must be fitted into the continuous solution using the shock-shock jump conditions,

$$
\tan \left(\theta_{2}-\theta_{1}\right)=\frac{\left[\left(A_{1}^{2}-A_{2}^{2}\right)\left(M_{2}^{2}-M_{1}^{2}\right)\right]^{1 / 2}}{A_{1} M_{1}+A_{2} M_{2}}
$$

and

$$
\tan \left(\chi-\theta_{1}\right)=\frac{A_{1}}{M_{1}}\left[\frac{M_{2}^{2}-M_{1}^{2}}{A_{1}^{2}-A_{2}^{2}}\right]^{1 / 2}
$$

where

$$
A=\exp \left\{-\int \frac{m \lambda(m)}{m^{2}-1} d m\right\}
$$

The subscripts 1 and $Z$ denote the quantities ahead of and behind the shockshock respectively, and $\chi$ is the angle of the shock-shock with the $x$-axis. The integration of the function $A$ is given in Appendix A. 
2.4.2. The contact discontinuity. For shock dynamics in a non-uniform medium, one must also account for the possible occurrence of discontinuities in fluid properties, that is, contact discontinuities, ahead of the shock front. We consider an element of interface inclined at an angle $\delta_{I}$ to the $x$-axis, as shown in figure 2.3. To remove the $x$-dependence from the source terms, we introduce a simple rotation of the shock and the interface through an angle $-\delta_{I}$, so that the interface lies parallel to the $x$-axis. Then $a_{0} \gamma$ and $p_{0}$ become functions of $y$ only, and equations (2.3.17) reduce to

$$
d \tilde{\theta} \pm d \omega=-\frac{1}{\tan \tilde{\theta} \pm \nu}\left[(1 \pm \nu g \tan \widetilde{\theta}) \frac{d a_{0}}{\alpha_{0}} \pm \nu \tan \tilde{\theta}\left[f d \gamma+h \frac{d p_{0}}{p_{0}}\right)\right]
$$

on the characteristics

$$
\frac{d y}{d x}=\frac{\tan \tilde{\theta} \pm \nu}{1 \mp \nu \tan \tilde{\theta}}
$$

where $\tilde{\theta}$, the angle between the rays of the local shock front and the interface, is given by

$$
\tilde{\theta}=\theta-\delta_{I}
$$

If we apply equations (2.4.5) to a pair of characteristics which intersects a contact interface across which there are infinitesimal changes of sound speed $d a_{0}$. specific heat ratio $d \gamma$, and pressure $d p_{0}$, on eliminating the terms in $a_{0}$, we obtain the simple result

$$
\tan \tilde{\theta} d \tilde{\theta}=-\frac{d U}{U}
$$




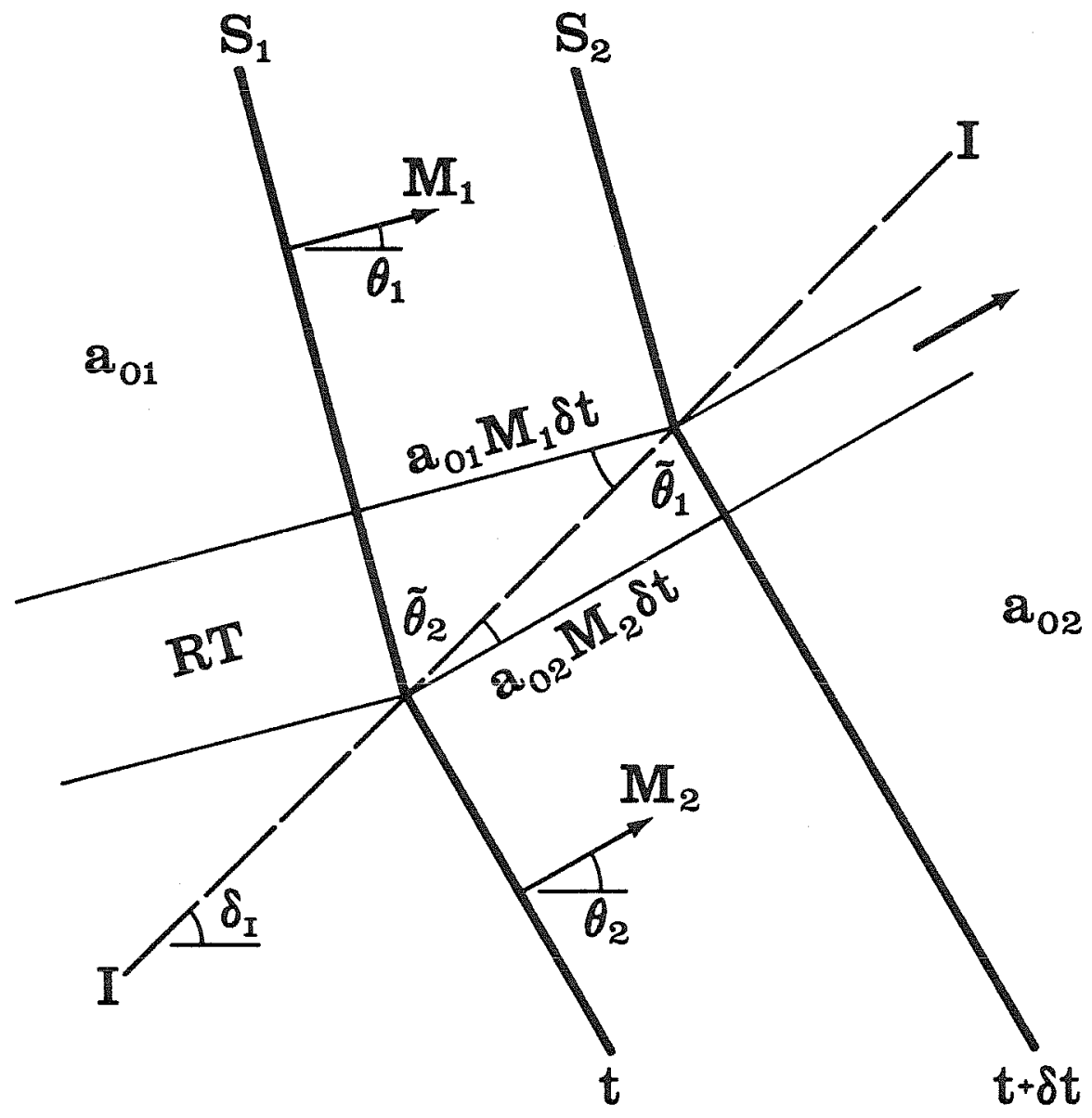

FIGURE 2.3. The contact surface discontinuity, $S_{1}, S_{2}$, shock front positions at two successive times, $t$ and $t+\delta t$; 1 , interface; $\mathrm{RT}$, ray tube. 
Then, assuming that the effects of an interface carrying finite changes can be built up by summing the infinitesimal changes, equation (2.4.7) is integrated to yield

$$
\frac{U}{\cos \ddot{\theta}}=\text { constant }
$$

Hence, equations (2.4.5) provide exactly the condition required for a continuous shock front across such an interface, a condition which is analogous to Snell's law of refraction.

By eliminating the terms in $\widetilde{\theta}$ from equations $(2.4 .5)$, we obtain

$$
\left(\tan ^{2} \tilde{\theta}-\nu^{2}\right) \frac{d M}{M}=\nu^{2}\left[\left(1-g \tan ^{2} \tilde{\theta}\right) \frac{d \alpha_{0}}{\alpha_{0}}-\tan ^{2} \tilde{\theta}\left(f d \gamma+h \frac{d p_{0}}{p_{0}}\right)\right] .
$$

In order to demonstrate the properties of the equations in the simplest way possible, we consider in the remainder of this work, the case $\gamma_{1} p_{0}=$ constant, that is, the medium is a perfect gas in which only variations of temperature occur. Then the last two terms in equations $(2,4.9)$ vanish and the equation reduces to an ordinary differential equation in $M$ and $a_{0}$,

$$
\frac{d M}{d a_{0}}=\frac{\nu^{2} M\left(U^{2}-g V^{2}\right)}{a_{0}\left(V^{2}-\nu^{2} U^{2}\right)}
$$

where

$$
U=a_{0} M
$$

and

$$
V=\left[\left(\frac{a_{01} M_{1}}{\cos \widetilde{\theta}_{1}}\right]^{2}-\left(\alpha_{0} M\right)^{2}\right]^{1 / 2}
$$


Given the shock strength and sound speed on one side of the interface, say $M_{1}$ and $a_{01}$, the ordinary differential equation $(2,4.10)$ can be solved to yield the shock strength $M_{2}$ on the other side, where the sound speed is $a_{02}$. To complete the solution of the jump conditions across the interface, the slope of the shock front in the secondary region is found from equation (2.4.8). Then, the final step is to restore the shock and the interface to their original positions by another simple rotation through an angle $+\delta_{I}$.

We note that the ordinary differential equation (2.4.10) is well-behaved except for a singularity which occurs when the denominator vanishes. It is shown in Appendix $\mathrm{C}$ that when the equation is singular, a characteristic lies parallel to the interface. It is also shown that the singularity is a square-root singularity, and that it is integrable. The physical significance of this condition is discussed in section 3.1 .

It is interesting to consider the direction of crossing of the ray tubes at the interface. Figure 2.3 has been drawn to illustrate the results of shock refraction for the case of $\theta<\delta_{I}$, and shown in the figure is a ray tube consisting of a bundle of rays. For the case of $\theta<\delta_{I}$, the ray tubes cross the interface from region 1 to region 2 , while for the case of $\theta>\delta_{I}$, the ray tubes cross the interface in the opposite direction. Since the ray tubes represent the channelling of energy between rays, the direction in which the ray tubes cross the interface indicates the region into which the refracted shock is growing. When $\theta=\delta_{I}$, the shock is normal to the interface and the ray tubes do not cross the interface. This condition is identical to that which is applied at a solid boundary in the analysis of shock diffraction using the theory of shock dynamics in a uniform medium. 


\section{Chapter 3}

\section{SHOCK REFRACTION}

As a demonstration of the application of the theory, we consider problems involving a plane shock wave incident at some angle on a free plane gaseous interface across which there exists a finite difference in sound speed (Jahn, 1956; Abd-el-Fattah, Henderson and Lozzi, 1976). Two different problems are discussed. In the first problem, only a gaseous interface is present, while the second involves a solid boundary as well. The problems are designed to be selfsimilar, and they have been chosen for consideration because non-simple regions do not appear in the flow-field. For regular refraction, the effects of the interface are only local, so in fact, regular refraction can be solved exactly by three-shock theory. However, shock dynamics also predicts irregular refraction, so even these simple cases are interesting.

As the first example, Problem 1, we choose the simplest configuration that contains all the important effects, namely, one in which the contact interface is wedge-shaped, with one of the sides aligned parallel with the undisturbed shock front and the other lying at an angle $\delta_{I}$ with the $x$-axis, as shown in figure 3.1 . $S_{1}$ is the incident shock front and $S_{2}$ is the shock front at a subsequent time after interaction with the interface. The shock transmits through the vertical portion of the contact surface and refracts from the other. Regions 1 through 6 in figure 3.1 are uniform regions. The shock in region 2 provides the lower boundary condition in this problem, and is calculated exactly from the equations of one-dimensional gasdynamics (Appendix D), while in regions $3,4,5$ and 6 , the shock conditions are calculated from the approximate theory developed here. 


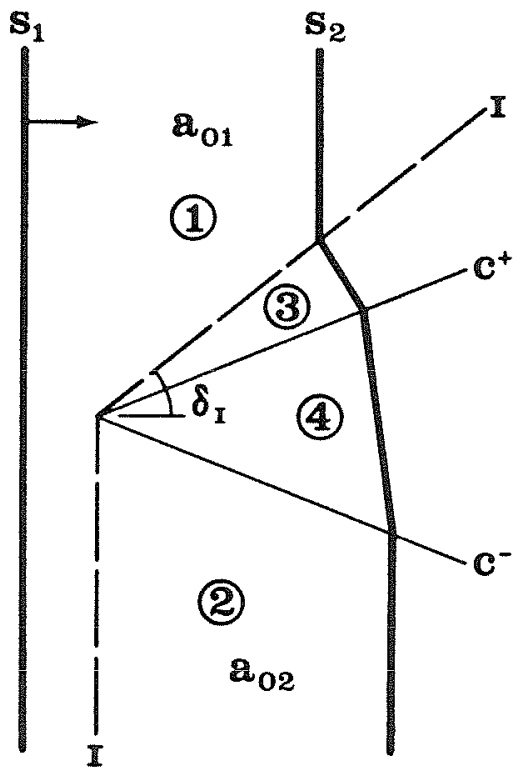

(a)

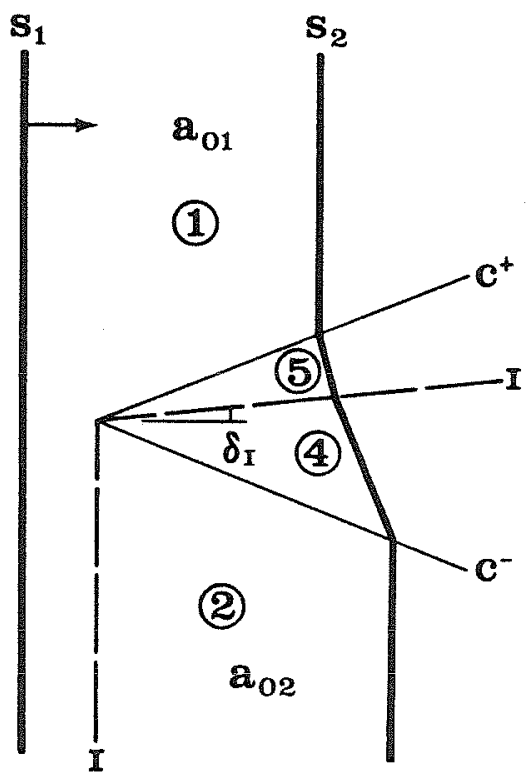

(b)

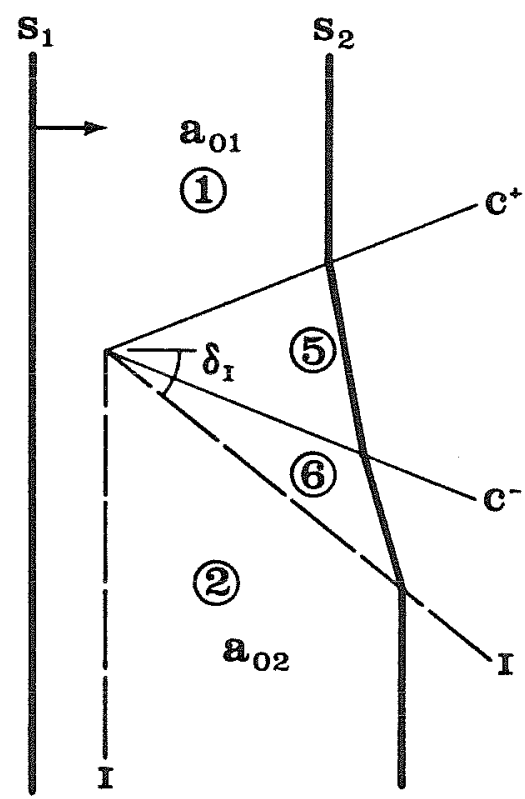

(c)

FIGURE 3.1. Configurations for Problem 1; (a) Contact surface above leading $C^{+}$characteristic; (b) Contact surface between leading $C^{+}$and $C^{-}$characteristics; (c) Contact surface below leading $C^{-}$characteristic. $S_{1}$, incident shock front: $S_{2}$, shock front after interaction with interface; $I$, interface. 
In the second example, Problem 2 , a solid wall is introduced below the sloping interface, at an angle $\delta_{\mathbb{y}}$ with the $x$-axis, as shown in figure 3.2 , and the boundary condition imposed here is that the shock is always normal to the wall. Regions 1 through 4 are uniform and they are calculated from shock dynamics theory.

As shown in figures 3.1 and 3.2, different cases are possible for each problem, depending on the position of the interface with respect to the leading $C^{+}$and $C^{-}$characteristics. In Problem 1, the interface may lie above the leading $C^{+}$ characteristic, it may lie between the leading $C^{+}$and $C^{-}$characteristics, or it may lie below the leading $C^{-}$characteristic. Likewise, in Problem 2 , the interface may lie above the leading $C^{+}$characteristic, or it may lie between the leading $C^{+}$characteristic and the wall. When the interface lies above the leading $C^{+}$characteristic or below the leading $C^{-}$characteristic, the steepness of the interface prevents disturbances on the shock front from propagating outward from the interface, and these are cases of regular refraction. However, in Problem 1, when the interface lies between the leading $C^{+}$and the leading $C^{-}$ characteristics, or in Problem 2, when it lies between the leading $C^{+}$characteristic and the wall, the disturbances propagate outward from the interface, resulting in irregular refraction. Thus, shock dynamics theory for a nonuniform medium models both regular and irregular refraction. This is not the case for shock dynamies in a uniform medium, where, for the reflection of a shock by a solid wedge, the theory predicts a very tiny Mach stem for conditions under which regular reflection actually occurs (Whitham, 1957).

In order to demonstrate the quantitative behaviour of the theory, shock refraction has been calculated for several different values of $\delta_{I}, \delta_{W}, a_{0}$ and $M$. The case $a_{02}>\mathbb{a}_{01}$ (the "slow-fast" interaction) has been investigated in detail for both problems, while the case $a_{02}<\alpha_{01}$ (the "fast-slow" interaction) has so 


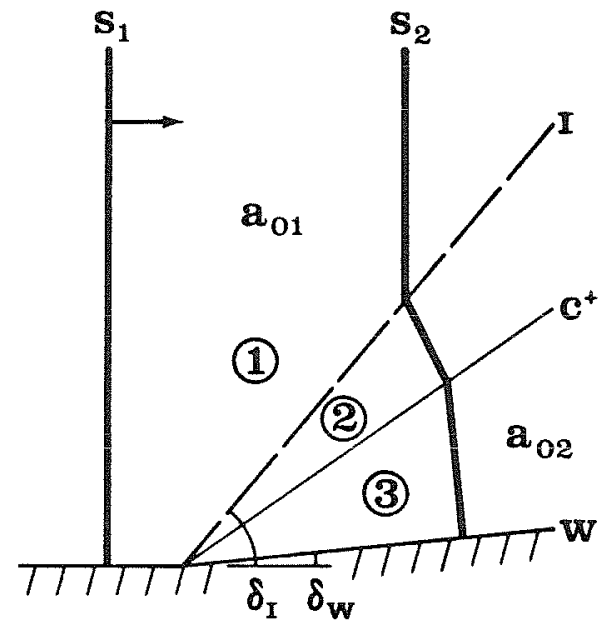

(a)

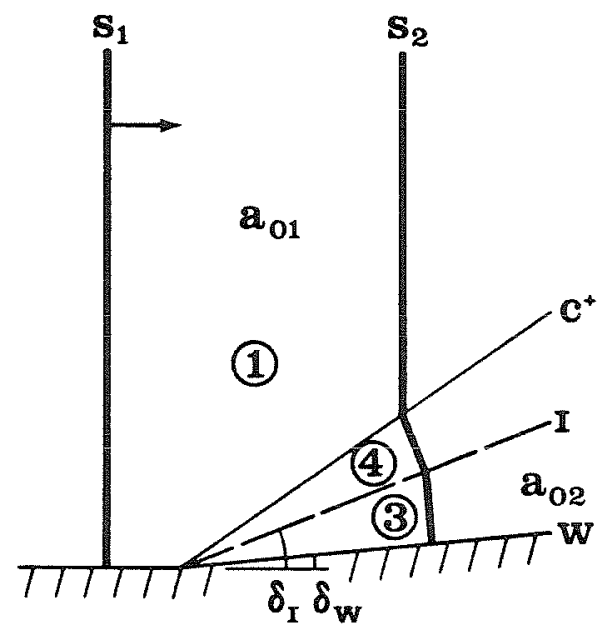

(b)

FIGURE 3.2. Configurations for Problem 2; (a) Contact surface above leading $\mathrm{C}^{+}$characteristic; (b) Contact surface between leading $\mathrm{C}^{+}$and wall: $S_{1}$, incident shock front; $S_{2}$, shock front after interaction with interface; $I$, interface; $W$, wall. 
far been treated only for Problem 1. Typical results are presented in tables 3.1 and 3.2 .

The problems are solved using the interface jump conditions (equations (2.4.8) and (2.4.10)), the shock-shock jump conditions (equations (2.4.2) and (2.4.3)), and the invariance of $\theta \pm \omega$ in the uniform regions and in the simple waves. All the calculations were done numerically, including the integration of equation (2.4.10), for which a fourth-order Adams-Moulton predictor-corrector algorithm was used, started off by the Runge-Kutta-Gill method. For the regular refraction cases, the conditions behind the interface are determined directly from the known conditions ahead of the interface, and the remaining region is then determined from the boundary conditions. However, for the irregular refraction cases, since none of the conditions in the two regions adjacent to the interface is known a priori, it is necessary to make a guess of one quantity, say the Mach number, $M$, ahead of the interface and then to solve the equations iteratively. Further details are contained in Appendix E. Unless otherwise stated, the computational accuracy for $M$ in these cases is better than $0.001 \%$.

Although the theory of shock dynamics in a non-uniform medium is approximate and provides information only about the main shock front, it has an important advantage over existing methods for calculating shock refraction at a gaseous interface, in that it is simple to apply and yields a unique solution. On the other hand, solution of the full gasdynamics equations from three-shock theory (Taub, 1947; Polachek and Seeger, 1951; Henderson, 1966 and others), yields information about the reflected wave as well, but, in general, there are twelve possible roots, so that it is necessary to develop a criterion to determine which solution agrees best with experiment. This has been the source of considerable controversy, 


\subsection{Problem 1}

A series of solutions for Problem 1 with $M_{1}=5.0$ and $a_{02} / a_{01}=2.0$ is given in table 3.1, and some typical cases are illustrated in figure 3.3. The uniform regions ( 1 through 6) are adjacent to simple waves (shock-expansions), or to discontinuities (shock-shocks or the contact surface). The shock front is continuous throughout, but the slope is discontinuous at the shock-shock and at the contact surface. Both the shock strength and slope vary continuously through the shock-expansions. As might be expected, for the slow-fast interaction the theory shows the shock to be concave forward in the upper part of the flow field and convex in the lower part.

Table 3.1. Summary of calculated refraction parameters for Problem 1

$$
\begin{gathered}
M_{1}=5.0, \theta_{1}=0^{\circ} \text { and } a_{02} / a_{01}=2.0 \\
\left(M_{2}=3.43 \text { and } \theta_{2}=0^{\circ}\right)
\end{gathered}
$$

\begin{tabular}{c|ccccccccccc} 
Case & $\delta_{I}$ & $M_{3}$ & $\theta_{3}$ & $M_{4}$ & $\theta_{4}$ & $M_{5}$ & $\theta_{5}$ & $M_{6}$ & $\theta_{6}$ & $\chi_{1}$ & $\chi_{2}$ \\
\hline 1 & 90.0 & 3.56 & 0.0 & 3.49 & -2.3 & - & - & - & - & - & - \\
2 & 60.0 & 3.73 & 18.3 & 3.34 & 3.6 & - & - & - & - & - & - \\
3 & 55.7 & $(4.07)$ & $(32.2)$ & 3.31 & 4.9 & - & - & - & - & - & - \\
4 & 55.0 & - & - & 3.29 & 5.7 & $(8.71)$ & $(52.3)$ & - & - & 55.0 & - \\
5 & 45.0 & - & - & 3.16 & 11.1 & 7.44 & 42.5 & - & - & 48.0 & - \\
6 & 36.8 & - & - & 3.10 & 13.6 & 6.74 & 34.4 & - & - & 42.8 & - \\
7 & 30.0 & - & - & 3.06 & 15.4 & 6.31 & 28.2 & - & - & 38.9 & - \\
8 & 20.5 & - & - & 2.94 & 20.5 & 5.89 & 20.5 & - & - & 34.6 & - \\
9 & 15.0 & - & - & 2.78 & 28.1 & 5.70 & 16.6 & - & - & 32.4 & - \\
10 & 13.8 & - & - & $(2.61)$ & $(36.6)$ & 5.67 & 15.9 & - & - & 32.0 & - \\
11 & 14.0 & - & - & - & - & 5.66 & 15.7 & $(3.01)$ & $(71.9)$ & 31.9 & 14.0 \\
12 & 0.0 & - & - & - & - & 5.40 & 10.0 & 3.39 & 57.3 & 28.8 & 6.1 \\
13 & -23.3 & - & - & - & - & 5.21 & 5.2 & 4.13 & 33.1 & 26.4 & -5.6 \\
14 & -45.0 & - & - & - & - & 5.12 & 3.0 & 4.60 & 16.7 & 25.3 & -14.1 \\
15 & -71.0 & - & - & - & - & 5.00 & 0.0 & 4.78 & 5.9 & 23.7 & -20.8
\end{tabular}

Angles $\delta, \theta$ and $\chi$ are given in degrees.

Values in parentheses denote vanishingly small regions. 


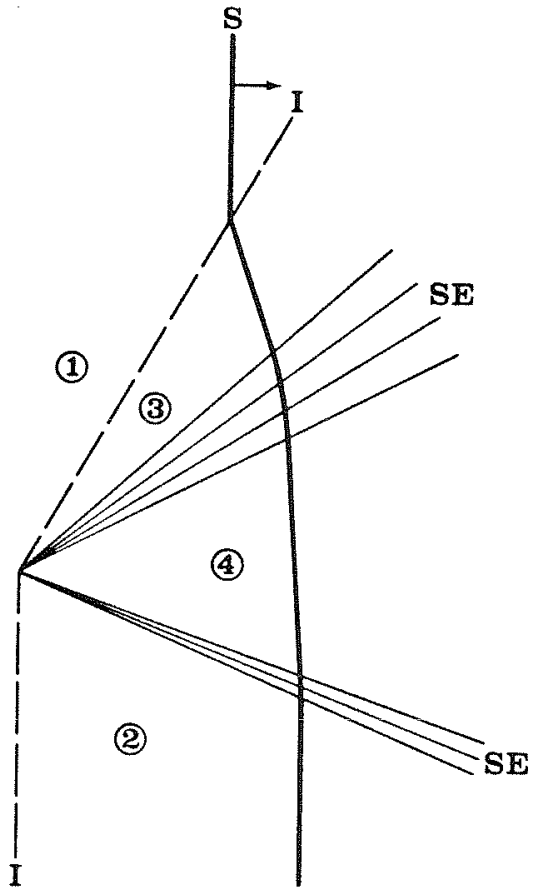

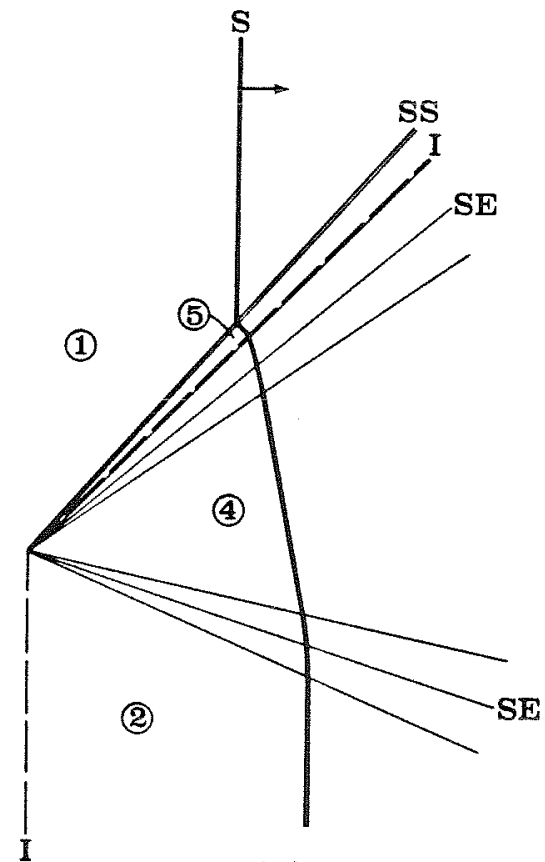

(b)

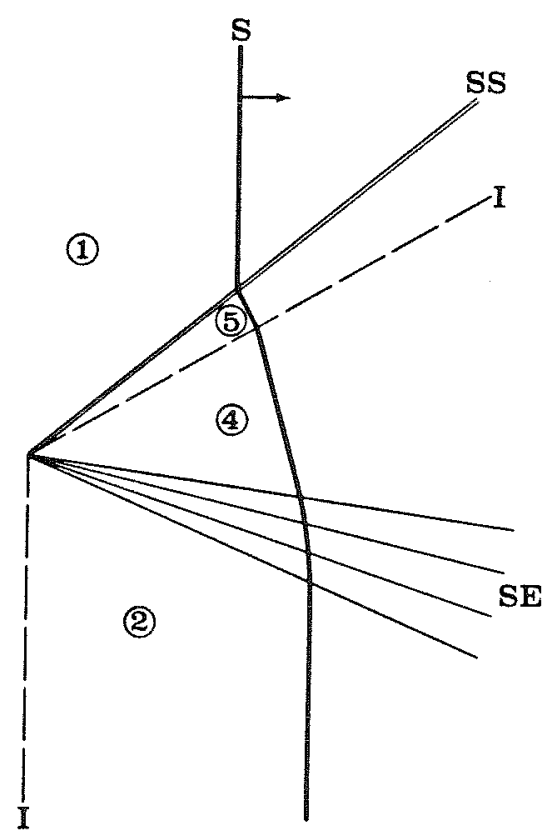

(c)

FIGURE 3.3. Solutions for Problem 1 with $M_{1}=5.0$ and $a_{02} / a_{01}=2.0$; (a) Regular refraction, $\delta_{I}=60.0^{\circ}$ (case 2); (b) Irregular refraction, $\delta_{I}=45.0^{\circ}$ (case 5); (c) Irregular refraction, $\delta_{I}=30.0^{\circ}$ (case 7); S, shock front; I, interface; SE, shock-expansion; SS, shock-shock. 


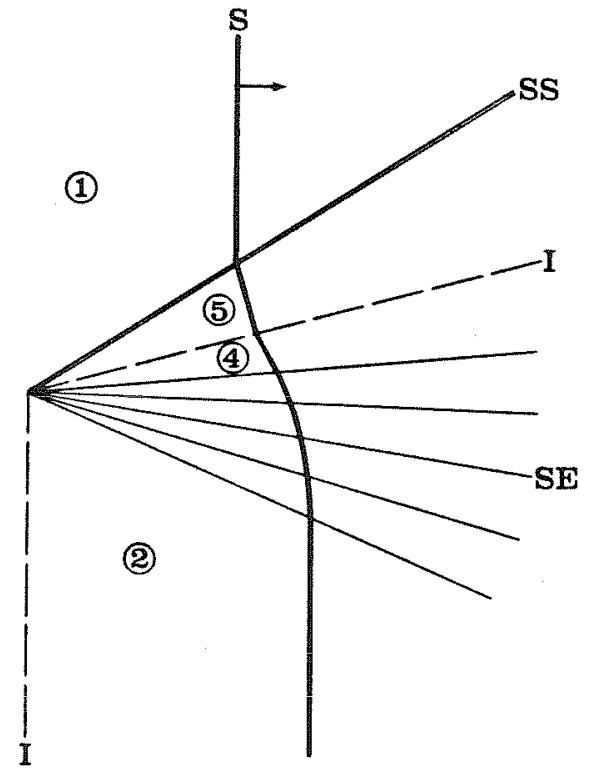

(d)

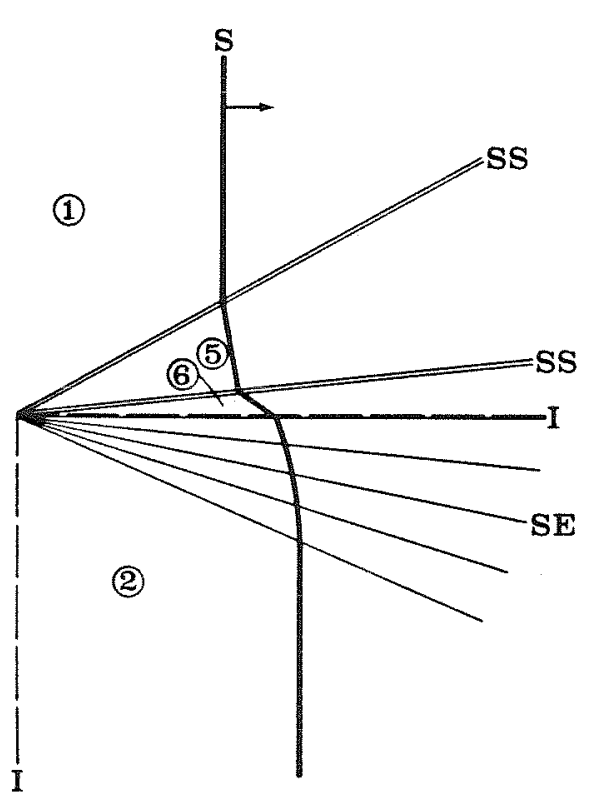

(e)

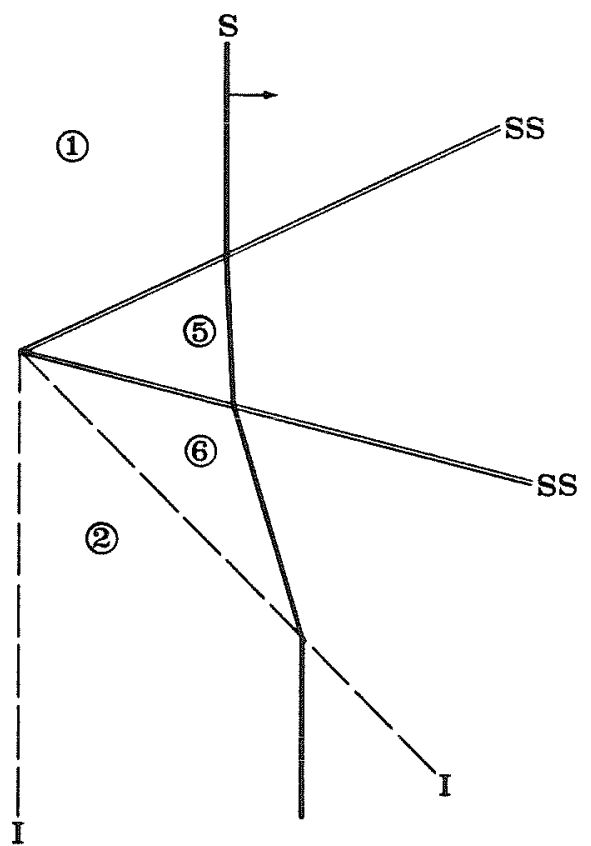

(f)

FIGURE 3.3. (Continued) Solutions for Problem 1 with $M_{1}=5.0$ and $a_{02} / \alpha_{01}=2.0_{i}$ (d) Irregular refraction, $\delta_{I}=15.0^{\circ}$ (case 9 ); (e) Irregular refraction, $\delta_{I}=0^{\circ}$ (case 12); (f) Regular refraction, $\delta_{I}=-45.0^{\circ}$ (case 14 ). $\mathrm{S}$, shock front; I, interface; SE, shock-expansion; SS, shock-shock. 
For large interface angles, a regular refraction solution is obtained, which contains two shock-expansions of opposite families, as shown in figure 3.3(a) (case 2). Shock dynamics theory predicts transition from regular to irregular refraction when the slope of the interface is equal to the slope of the characteristics in the region just behind the interface. This occurs when the uppermost characteristic of the shock-expansion between regions 3 and 4, designated here as $C_{t}^{+}$, approaches the contact surface, and region 3 vanishes. Then,

$$
\delta_{I_{t}^{+}}=\left.\tan ^{-1}\left(\frac{d y}{d x}\right)\right|_{c_{t}^{+}}
$$

where $\delta_{I_{t}^{+}}$is the interface angle at transition, and $d y / d x$ is given by equation (2.3.1B), evaluated on $C_{t}^{+}$(case 3 ). We note that at transition, the flow deflection angle is greatest, and designate this angle $\theta_{3_{i}}$.

Up to this point, the $C^{+}$characteristics crossing the interface originate in region 1, and the direction of crossing is from above to below. However, a further decrease in $\delta_{I}$ results in a reversal in the direction of crossing of the $C^{+}$characteristics, which now originate from the corner. The $C^{+}$characteristics emerging from the interface are steeper than those in region 1, and this results in the formation of a shock-shock discontinuity, which initially lies just above the interface (case 4 ), at an angle $\chi_{1}$ with the $x$-axis. As the interface angle is decreased further, the shock-shock separates from the interface, as shown in figure 3.3(b) (case 5), and the upper shock-expansion becomes smaller, until it finally vanishes (case 6).

In cases 4 through 6 , and in other cases to be encountered later, a characteristic lies just behind and adjacent to the interface. Under these conditions, a singularity appears in the expression for $d M / d \alpha_{0}$ (equation (2.4.10)), as $a_{0}$ 
approaches $a_{02}$. It is shown in Appendix $C$ that the singularity is a square-root singularity, so that $d M / d a_{0}$ is integrable, and solutions exist for this range of interface angles.

Transition from regular to irregular refraction in shock dynamics theory is analogous to that which occurs in the problem of a moving force field or 'leaky piston" in unsteady one-dimensional gasdynamics. In this problem, Hoffman (1967) found that a transition occurs from a shock-free solution, when the force is weak and supersonic, to a solution containing a shock, when the force is subsonic. In the analogy, the force, which is a "source" in gasdynamics, corresponds to the contact interface, which is a "source" in shock dynamics, and in particular, a weak supersonic force corresponds to a steeply inclined interface, while a subsonic force corresponds to a gradually sloping interface. Further, in an intermediate case, analogous to our cases 4 through 6 , Hoffman also found that a characteristic in the $(x, t)$ plane lies adjacent to the force and behind it, while the characteristics ahead, between the force and the shock, lie at a finite angle to the force. For this case, as in our result, a square-root singularity exists at the back of the discontinuity. The appearance of a characteristic adjacent to and at the back of a surface of discontinuity signifies a sonic condition well-known in gasdynamics, a familiar example of which is Chapman-Jouget detonation. The physical processes leading to this case are most easily understood for a force field (or contact region) of finite width, further details of which can be found in Hoffman (1967). Our use of the differential relation, equation (2.4.10), is equivalent to treating the contact discontinuity as a region of finite width.

It is also interesting to note that there exists a finite range of interface angles $\left(0.7^{\circ}\right.$ in this instance, between cases 3 and 4$)$ in which no solution could be obtained for the irregular refraction case. It appears that this difficulty is 
associated with the formation of the shock-shock on the interface, and a similar difficulty occurs whenever a shock-shock lies on or immediately adjacent to the interface.

Shown in figure $3.3(c)$ is the geometry of a simple irregular refraction, after the upper shock-expansion has vanished (case 7). As the interface angle continues to decrease, the shock front becomes normal to the interface (case B) and then reverses curvature (case 9) from convex forward at the interface to concave forward, as shown in figure $3.3(\mathrm{~d})$. For case $B$, in which we designate the interface angle as $\delta_{I_{n}}$, we note that the shock fronts on both sides of the interface are normal to the interface, so that the ray tubes do not cross the interface. In other words, there is no transfer of energy across the interface, as discussed in section 2.4,2. This case is identical to the diffraction of a shock by a wedge of angle $\delta_{I}$.

For a further decrease in the slope of the interface, a second transition angle, $\delta_{I_{t}^{-}}$, is reached when the uppermost characteristic of the lower shock-expansion, $C_{l}^{-}$, approaches the contact surface and region 4 vanishes, that is,

$$
\delta_{I_{t}^{-}}=\left.\tan ^{-1}\left(\frac{d y}{d x}\right)\right|_{c_{l}^{-}} .
$$

where $d y / d x$ is evaluated on $C_{l}^{-}$(case 10). For $\delta_{I}<\delta_{I_{t}^{-}}$, the behaviour of the $C^{-}$characteristics is similar to the behaviour of the $C^{+}$characteristics for $\delta_{I}<\delta_{I_{t}^{+}}$The $C^{-}$characteristics which cross the interface now originate from the corner, instead of originating in region 1 as before, and the direction of crossing is now from below to above. The steepness of the $\mathrm{C}^{-}$characteristics emerging from the interface results in the formation of a second shock-shock, which initially lies just above the interface (case 11), at an angle $\chi_{2}$ with the $x$ - 
axis. As shown in figure 3.3(e) (case 12), the shock-shock separates from the interface as the interface angle is decreased, and the lower shock-expansion becomes smaller, until it finally vanishes (case 13).

At this point (case 13), the interface lies on the leading $C^{-}$characteristic. When the interface lies below this characteristic, no information about the corner can propagate outward along the shock from the interface, so that the shock refraction at the interface becomes locally regular. A typical example is shown in figure 3.3(f) (case 14), in which we see that two shock-shocks of opposite families are present, but no shock-expansions.

This behaviour continues as the interface moves down toward the vertical. Both shock-shocks weaken, and as expected, the shock front straightens out and its strength tends to the undisturbed value of $M_{1}$. However, in region 6 , where the shock front has passed through two interfaces, the recovery is incomplete, and this may be attributed to reflection losses at each of the two interactions.

The results of this analysis show that if the interface is steep enough, the only mechanism by which concave curvature ("compressive" bending) can be induced on the shock is that provided by Snell's law, at or within the interface (cases 2 and 3 ). However, for smaller interface angles, when the refraction is irregular, convex curvature ("expansive" bending) occurs at the interface, presumably because too much compressive bending occurs at the shock-shock (cases 4 through 7). For even smaller interface angles, the shock-shock may be sufficiently weak so that the effects of the interface are once again compressive (cases 9 and 10), and the cycle repeats itself through the formation of a second shock-shock (cases 11 through 15). 
The solutions for this problem that involve two shock-expansions or two shock-shocks of opposite families are unusual, especially the latter, in which both the shock-shocks share a common Mach stem. Such solutions are the result of the unique properties of the characteristics in shock dynamics. The difference in behaviour between the characteristics in shock dynamics and the characteristics in gasdynamics is most easily seen from the fact that, in shock dynamics, the shock front, which is a locus of constant time, has no analogy in gasdynamics. It will be interesting to compare these predicted solutions with actual experimental results, when such results become available.

Before leaving this problem, we note that although we have been considering the slow-fast interface, the preceding results embody the form of the solutions for the fast-slow interface as well. For the fast-slow interface, we have $a_{02}<a_{01}$, $M_{2}>M_{1}$ and $U_{2}<U_{1}$, so that by interchanging region 1 with region 2 , we get $a_{02}>a_{01}, M_{2}<M_{1}$ and $U_{2}>U_{1}$, which are exactly the boundary conditions for the slow-fast interface. Of course, to get the detailed results for the fastslow case, it is necessary to determine $M_{2}$ at the vertical part of the interface, and then to solve for the various regions as before.

\subsection{Problem 2}

Given in table 3.2 and shown in figures 3.4 and 3.5 are some typical results for Problem 2. For any given incident shock strength and sound speed ratio, two different sequences of solutions are obtained, depending on the wall angle, $\delta_{\mathbb{B}}$. We note from the transition condition in Problem 1 (case 3 ), that when the interface angle is $\delta_{I_{l}^{+}}$, the flow deflection for regular refraction is maximum $\left(\theta_{3_{t}}\right)$, and that, in order to obtain a larger deflection, the flow adjusts itself by becoming irregular. Hence, for problem 2 , in which a wall is present below the interface, if $\delta_{\not F}>\theta_{3_{t}}$, for $\delta_{I}$ decreasing from $90^{\circ}$, it might be expected that the solution will become irregular before $\delta_{I}$ reaches $\delta_{I_{t}^{+}}$. Indeed, for $\delta_{\mathbb{T}}<\theta_{3_{t}}$, 
Table 3.2. Summary of calculated refraction parameters for Problem 2

\begin{tabular}{l|cccccccc}
\multicolumn{7}{c}{$a_{02 / a_{01}=2.0, M_{1}=5.0 \text { and } \delta_{W}=15.0^{\circ}}$} \\
Case & $\delta_{I}$ & $M_{2}$ & $\theta_{2}$ & $M_{3}$ & $\theta_{3}$ & $M_{4}$ & $\theta_{4}$ & $\chi$ \\
\hline 16 & 65.0 & 3.65 & 13.1 & 3.70 & 15.0 & - & - & 37.5 \\
17 & 62.9 & 3.68 & 15.0 & 3.68 & 15.0 & - & - & $(38.5)$ \\
18 & 60.0 & 3.73 & 18.3 & 3.64 & 15.0 & - & - & - \\
19 & 55.7 & $(4.07)$ & $(32.2)$ & 3.57 & 15.0 & - & - & - \\
20 & 55.0 & - & - & 3.53 & 15.0 & $(8.71)$ & $(52.3)$ & 55.0 \\
21 & 45.0 & - & - & 3.25 & 15.0 & 7.44 & 42.5 & 48.0 \\
22 & 38.2 & - & - & 3.15 & 15.0 & 6.84 & 35.8 & 43.7 \\
23 & 30.0 & - & - & 3.05 & 15.0 & 6.31 & 28.0 & 38.9
\end{tabular}

\begin{tabular}{c|cccccccc}
\multicolumn{8}{c}{$a_{02} / a_{01}=2.0, M_{1}=5.0$ and $\delta_{7}=45.0^{\circ}$} \\
Case & $\delta_{I}$ & $M_{2}$ & $\theta_{2}$ & $M_{3}$ & $\theta_{3}$ & $M_{4}$ & $\theta_{4}$ & $\chi$ \\
\hline 24 & 60.0 & 3.73 & 18.3 & 4.64 & 45.0 & - & - & 56.3 \\
25 & 57.8 & $(3.81)$ & $(22.0)$ & 4.58 & 45.0 & - & - & 57.8 \\
26 & 57.3 & - & - & 4.52 & 45.0 & $(9.25)$ & $(55.2)$ & 57.3 \\
27 & 50.0 & - & - & 4.10 & 45.0 & 8.22 & 49.1 & 52.6
\end{tabular}

\begin{tabular}{l|cccccccc}
\multicolumn{8}{c}{$\alpha_{02} / \alpha_{01}=2.0, M_{1}=5.0$ and $\delta_{W}=60.0^{\circ}$} \\
Case & $\delta_{I}$ & $M_{2}$ & $\theta_{2}$ & $M_{3}$ & $\theta_{3}$ & $M_{4}$ & $\theta_{4}$ & $\chi$ \\
\hline 28 & 75.0 & 3.58 & 6.8 & 6.34 & 60.0 & - & - & 62.4 \\
29 & 64.2 & $(3.66)$ & $(13.7)$ & 5.74 & 60.0 & - & - & 64.2 \\
30 & 63.9 & - & - & 5.67 & 60.0 & $(11.36)$ & $(63.1)$ & 63.9 \\
31 & 61.0 & - & - & 5.36 & 60.0 & 10.72 & 61.2 & 62.2
\end{tabular}

\begin{tabular}{c|cccccccc}
\multicolumn{7}{c}{$\alpha_{02} / \alpha_{01}=2.0, \delta_{I}=30.0^{\circ}$ and $\delta_{H}=0^{\circ}$} \\
Case & $M_{1}$ & $M_{2}$ & $\theta_{2}$ & $M_{3}$ & $\theta_{3}$ & $M_{4}$ & $\theta_{4}$ & $\chi$ \\
\hline 32 & 5.0 & - & - & 2.75 & 0.0 & 6.29 & 27.72 & 38.7 \\
33 & 4.0 & - & - & 2.21 & 0.0 & 5.05 & 28.14 & 38.9 \\
34 & 3.0 & - & - & 1.67 & 0.0 & 3.80 & 28.81 & 39.0 \\
35 & 2.0 & - & - & 1.14 & 0.0 & 2.53 & 29.79 & 38.6 \\
36 & 1.8 & - & - & 1.05 & 0.0 & 2.27 & 29.96 & 38.3 \\
37 & 1.78 & - & - & 1.04 & 0.0 & 2.24 & 29.98 & 38.2 \\
38 & 1.77 & - & - & 1.04 & 0.0 & 2.23 & 29.99 & 38.2
\end{tabular}

Angles $\delta, \theta$ and $\chi$ are given in degrees.

Values in parentheses denote vanishingly small regions. 


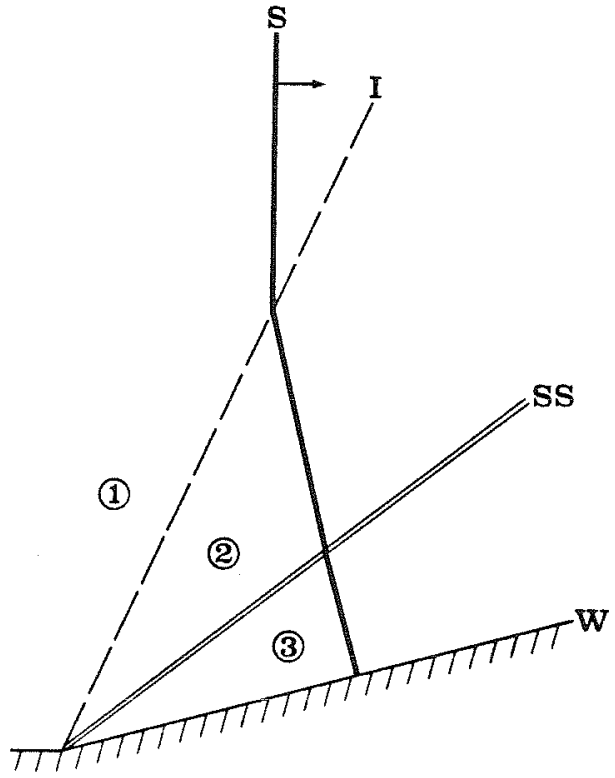

(a)

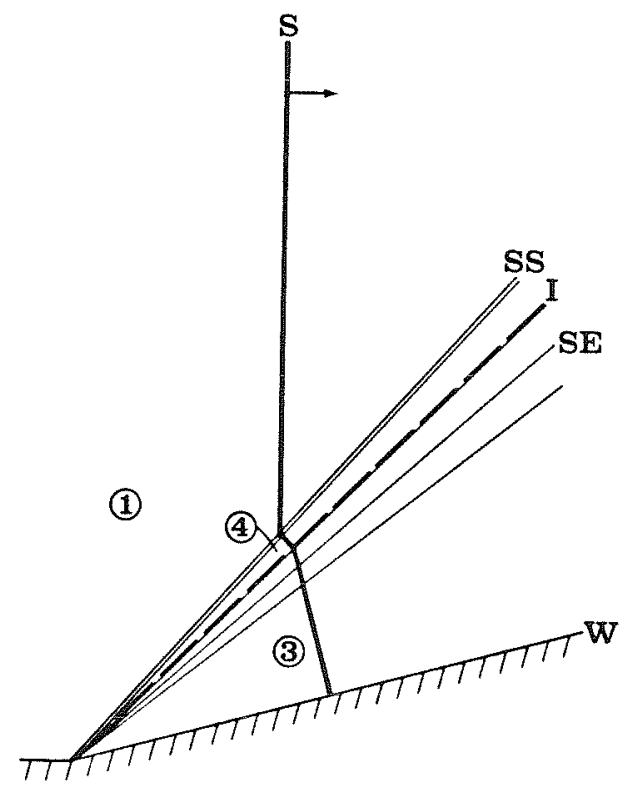

(c)

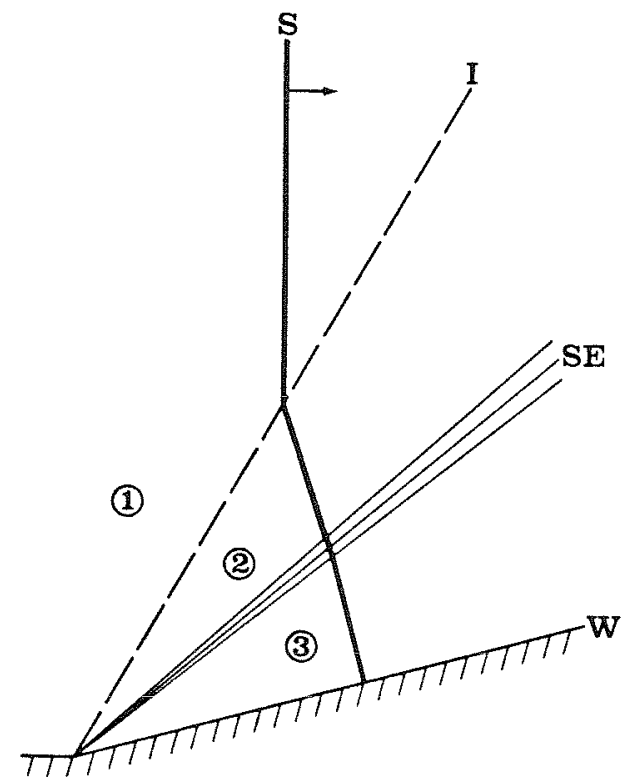

(b)

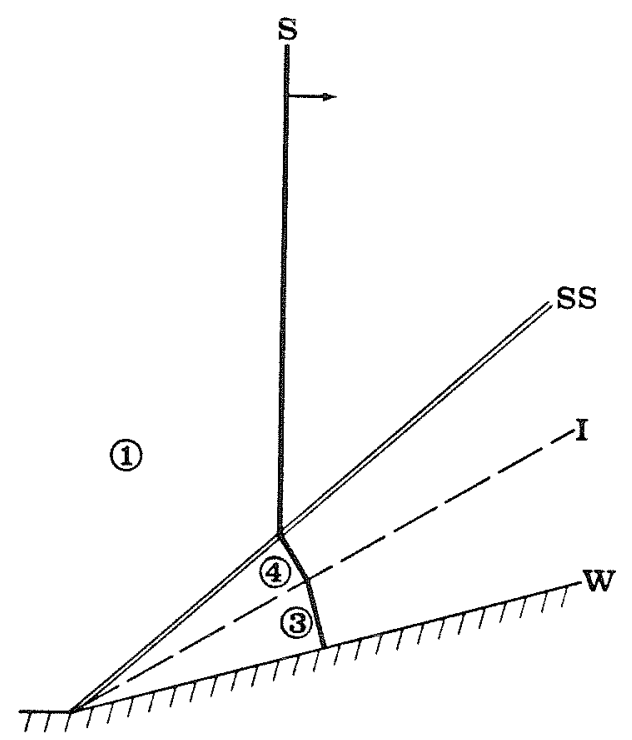

(d)

FIGURE 3.4. Solutions for Problem 2 with $M_{1}=5.0, a_{02} / a_{01}=2.0$ and $\delta_{y}=15.0^{\circ}$; (a) Regular refraction, $\delta_{I}=65.0^{\circ}$ (case 16); (b) Regular refraction, $\delta_{I}=60.0^{\circ} \quad$ (case 18); (c) Regular refraction, $\delta_{I}=45.0^{\circ}$ (case 21 ); (d) Irregular refraction, $\delta_{I}=30.0^{\circ}$ (case 23 ). S, shock front; I, interface; W, wall; SE, shock-expansion; SS, shock-shock. 


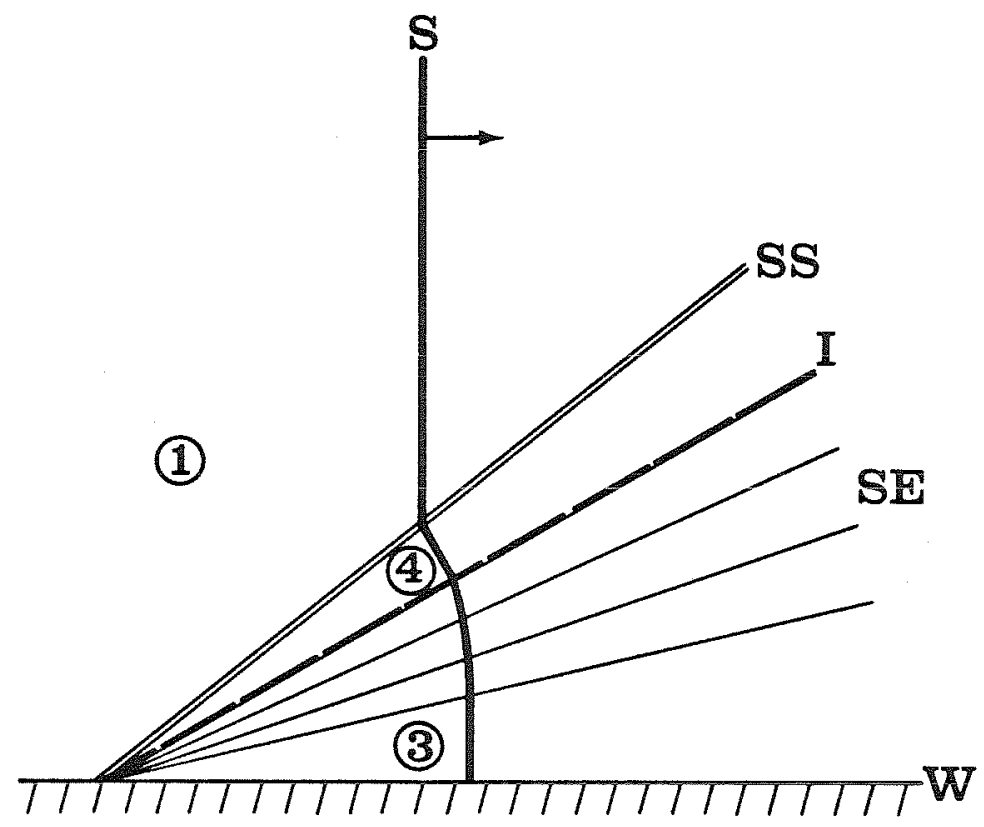

FIGURE 3.5. Irregular refraction solution for Problem 2 with $M_{1}=2.0, \quad a_{02} / a_{01}=2.0, \quad \delta_{W}=0^{\circ}$ and $\delta_{I}=30.0^{\circ}$ (case 35). S, shock front; I, interface; W, wall; SE, shockexpansion; SS, shock-shock. 
transition from regular to irregular refraction occurs at $\delta_{I}=\delta_{I_{t}^{+}}$, whereas for $\delta_{W}>\theta_{s_{t}}$, it occurs earlier. As a consequence, there are two sequences to be considered. An interesting third sequence is obtained by keeping the interface and wall angles fixed, and observing the effects of reducing the incident shock strength.

In the first and second sequences, we set $M_{1}=5.0$ and $a_{02} / \alpha_{01}=2.0$. For large interface angles and small wall slopes $\left(\delta_{w}<\theta_{3_{t}}\right)$, a shock-shock lies below the interface, as shown in figure 3.4(a) (case 16). As the interface angle is decreased, the shock-shock weakens, degenerates to a $C^{+}$characteristic (case 17), and is converted into a shock-expansion as shown in figure $3.4(\mathrm{~b})$ (case 18). The transition from the shock-shock to the shock-expansion results in regions 2 and 3 becoming identical (case 17), and corresponds to a zero contribution from the corner signal, referred to by Jahn (1956). In figure 3.4(a) (csse 16), the corner signal is a net compression, while in figure $3.4(\mathrm{~b})$ (case 1B), it is a net rarefaction.

As the interface angle is decreased further, the shock-expansion grows in size and strength, until its upper $C^{+}$characteristic lies adjacent to the interface (case 19). A further decrease in the interface angle results in the formation of a shock-shock just above the interface, that is, irregular refraction (case 20), and as the interface angle becomes even smaller, the shock-shock separates from the interface as shown in figure $3.4(c)$ (case 21 ), the shock-expansion vanishes (case 22) and, finally, a simple irregular refraction results, as shown in figure $3.4(\mathrm{~d})$ (case 23 ). In the limit, as the interface collapses onto the wedge $\left(\delta_{I} \rightarrow \delta_{W}\right)$, the problem reduces to that of the irregular reflection of a shock by a wedge. 
It is interesting to note that for the special case of the horizontal wall $\left(\delta_{W}=0^{\circ}\right)$, by considering the wall to be an axis of symmetry, one obtains the solution to the double wedge problem, in which both faces of the gaseous wedge are inclined at equal angles to the undisturbed rays of the incident shock wave.

The second sequence of solutions occurs when the wall angle is large $\left(\delta_{\mathbb{W}}>\theta_{3_{t}}\right)$. For large interface angles, as before, a shock-shock lies below the interface, between regions 2 and 3 (case 24). However, as the interface angle is decreased, the shock-shock moves towards the interface (case 25), crosses it (case 26), and moves out into the primary medium, resulting in a simple irregular refraction result (case 27). The shock-shock is not converted into a shockexpansion, and the corner signal is compressive for all interface angles. In view of the fact that the order of crossing of the interface and the shock-shock by the $C^{-}$characteristics is reversed when the shock-shock passes through the interface, cases 25 and 26 , it is remarkable that the conditions in region 3 nevertheless show the expected trend.

The larger the wall angle, the nearer the shock-shock remains to the wall for all interface angles (cases $2 B$ through 31 ). Each of these solutions contains a tiny Mach stem, either at the wall (cases 28 and 29), or at the interface, when it is near the wall (cases 30 and 31 ). The Mach stem is the result of the boundary condition applied at the wall, and it is present even though the angles involved are large enough that the solutions should be regular. Thus, even in the limiting cases of $\delta_{I}=90^{\circ}$ and $\delta_{I}=\delta_{W}$, our solution predicts the presence of a Mach stem at the wall, instead of giving a regular refraction result.

In the third sequence, we set $\delta_{I}=30.0^{\circ}$ and $\delta_{W}=0^{\circ}$ (cases 32 through 38 ). As the incident shock strength is reduced, the strength of the shock at the wall, $M_{3}$ tends to unity, and the shock front in region 4 becomes normal to the 
interface, as shown in figure 3.5 (case 35 ). It appears that both these limits are reached simultaneously, which is not surprising, since the conditions at the interface are influenced by the conditions at the wall. Physically, as the limit is approached, the shock-expansion behind the interface, which has been expanding downwards, fills the secondary medium entirely, and in the limit, the ray tubes in region 4 do not cross the interface. If the incident shock strength were to be reduced, or alternatively, if the interface angle were to be decreased, the shock-expansion would have to expand further. This is not possible, and since the expansion cannot be discontinuous, it appears that this situation is the limiting case for which the equations at the interface, equations (2.4.8) and $(2.4 .10)$, and the boundary conditions can be satisfied simultaneously. This can be interpreted to mean that beyond this limit, the shock front should no longer be continuous across the interface. It has been observed experimentally that this situation leads to the formation of a precursor wave, that is, a discontinuous shock front at the interface, as discussed in section 4.1.2.

The essential difference between the case of a normal shock at the interface for Problem 1 (case B), and the limiting case of a normal shock here, lies in the behaviour of the ray tubes. In Problem 1, a portion of the shock front is present below the sloping part of the interface, having been transmitted through the vertical part, so that, as the interface angle passes through $\delta_{I_{n}}$, the direction in which the ray tubes cross the interface is simply reversed. However, in the case of Problem 2, the portion of the shock front below the interface comes only from the ray tubes which cross the interface from above. Thus, when the shock becomes normal to the interface, it appears that no further solution is possible by shock dynamies theory. 


\section{Chapter 4}

\section{COMPARISON WITH EXPERIMIENT AND THEORY}

In this chapter, the results from shock dynamics theory are compared with experimental and theoretical results. In particular, comparison is made with the experimental data of Jahn (1956), and with the experimental and theoretical work of Henderson's group (Abd-el-Fattah, Henderson and Lozzi, 1976; Abd-elFattah and Henderson, 1978). The simple case of normal interaction at an interface is also discussed.

\subsection{Comparison with experimental results}

4.1.1. Jahn's results. Jahn (1956) published experimental results on shock refraction at a plane gaseous interface. He used gas combinations of air methane and air - carbon dioxide, and the gases were prevented from mixing at the interface by a very thin plastic membrane. The results of computations based on the settings used in his experiments are presented in table 4.1, together with the experimental results which were measured from his published photographs. For the regular refraction case, according to shock dynamics theory, the conditions in region 3 are determined solely from the conditions in region 1, so that the comparison with experiment is only local. For the irregular refraction case, however, the conditions at the interface are influenced by the presence of the wall, so that it is necessary to consider the whole flow field.

In order to locate the shock-shock in the photographs, the position of the corner had to be determined by extending the lines along the interface and the wall upstream until they intersected. Since the angle between the interface and the wall is small in both cases 45 and 46 , this introduces a possible error in the 
Table 4,1. Calculated and measured refraction parameters based on settings used by Jahn

Regular Refraction
\begin{tabular}{c|ccc||c|c} 
Case & $\delta_{I}$ & $M_{1}$ & $\alpha_{02} / \alpha_{01}$ & $\begin{array}{c}\text { Theoretical } \\
\theta_{3}\end{array}$ & $\begin{array}{c}\text { Experimental } \\
\theta_{3}\end{array}$ \\
\hline 39 & 62 & 1.073 & 1.289 & 9.0 & 7 \\
40 & 47 & 1.073 & 1.289 & 19.1 & 19 \\
41 & 66 & 1.073 & 0.780 & -5.3 & -5 \\
42 & 27 & 1.073 & 0.780 & -19.3 & -19 \\
43 & 41 & 1.732 & 0.780 & -11.3 & -11 \\
44 & 32 & 1.732 & 0.780 & -15.8 & -15
\end{tabular}

Irregular Refraction - Problem 2
\begin{tabular}{c|cccc||cc|cc} 
Case & $\delta_{I}$ & $\delta_{W}$ & $M_{1}$ & $\alpha_{02} / a_{01}$ & $M_{3}$ & $\chi$ & $M_{3}$ & $\chi$ \\
\hline 45 & 35 & 25 & 1.732 & 1.289 & 1.70 & 39.5 & 1.69 & 37 \\
46 & 15 & 1 & 1.073 & 1.289 & 1.00 & 26.7 & 1.00 & 21
\end{tabular}

Angles $\delta, \theta$ and $\chi$ are given in degrees.

measured value for $\chi$. Further, in all four cases, the ratio $\gamma_{2} / \gamma_{1}$ is approximately 0.93 , whereas in the computations, $\gamma_{2} / \gamma_{1}$ has been taken to be unity. Nevertheless, it is seen from the table that the calculated values compare favourably with the values measured from the photographs.

Case 46, which is based on figure 14(e) of Jahn's paper, requires special comment. The experimental result has a discontinuous main shock front and a precursor wave at the interface. Shock dynamics, however, attempts to find a solution in which the main shock front is continuous across the interface. The solution for this case does not converge to within the specified tolerance, and the computational accuracy is only $1 \%$. Thus, although the result for case 46 is approximate, it shows the agreement of the theory with the experimental result, that under these conditions, the shock front is not continuous at the interface. 
4.1.2. Abd-el-Fattah, Henderson and Lozzi's results. Abd-el-Fattah, Henderson and Lozzi (1976) have performed a series of experiments on shock refraction using a set-up similar to that of Jahn. In order to obtain as large a sound speed ratio as possible, they used a carbon dioxide - helium gas combination across the interface. They observed that the gases leaked through the membrane, and using a gas analyser, they recorded that at the time the shock tube was fired, the carbon dioxide was about $95 \%$ pure ( $5 \%$ by volume helium) and the helium was about $90 \%$ pure (10\% by volume carbon dioxide).

Shown in figure 4.1 is the observed transmitted shock wave angle $\omega_{t}$ versus the incident shock wave angle $\omega_{i}$. The figure is reproduced from their paper, and the solid lines are their best fit curves for the experimental data points, $\oplus$ (regular refraction) and $\nabla$ (irregular refraction). The wave angles $\omega_{i}$ and $\omega_{t}$ are related to our notation by

$$
\begin{aligned}
& \omega_{i}=90-\delta_{I} \\
& \omega_{t}=\theta_{t}+\left(90-\delta_{I}\right)
\end{aligned}
$$

and

$$
\tilde{\chi}=\chi-\delta_{I}
$$

where $\theta_{t}$ is the ray angle of the transmitted shock front at the interface.

The results of our calculations have been entered as data points, (. These calculations are based on a contaminated carbon dioxide - helium interface, across which $a_{02} / \alpha_{01}=2.53$, but we have taken the ratio $\gamma_{2} / \gamma_{1}$ across the interface to be unity, instead of using the actual value of 1.22. Further, it has been assumed that the back plate is in line with the front plate, that is, $\delta_{W}=0^{\circ}$. Agreement with the experimental results is very good, and over the whole range of incident wave angles, from $\omega_{i}=0^{\circ}$ through transition, the present theory 


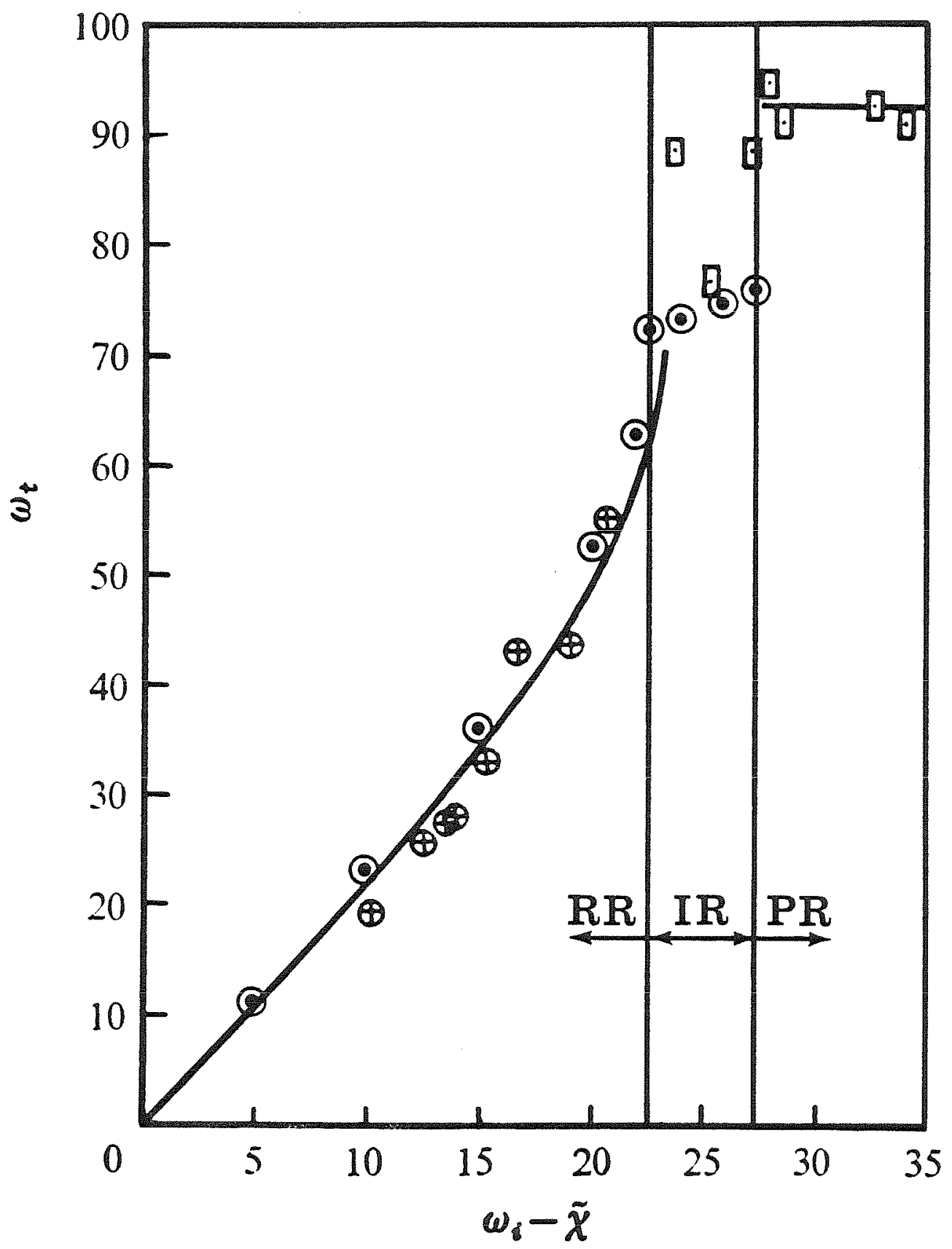

FIGURE 4.1. Transmitted shock wave angle $\omega_{i}$ versus incident shock wave angle $\omega_{i}$ for refraction of a plane shock at a contaminated carbon dioxide - helium interface. $\odot$, present theory; $\oplus$, experimental results (regular refraction); $\theta$, experimental results (irregular refraction); — Abd-El-Fattah, Henderson \& Lozzi's best fit curves for the experimental data; $R R$, regular refraction; IR, irregular refraction; PR, precursor irregular refraction. 
provides a much better model than either the Snell-piston theory or the pistondiaphragm theory of Abd-el-Fattah, Henderson and Lozzi (1976). We find that transition from regular to irregular refraction occurs at $\omega_{i}=22.7^{\circ}$, which compares with $\omega_{i}=23.12^{\circ}$ at transition from the three-shock theory, discussed in section 4.2 .

Over the range $22.7^{\circ}<\omega_{i}<27.6^{\circ}$, the refraction is irregular and a shockshock lies above the interface. (Between $\omega_{i}=22.7^{\circ}$ and $\omega_{i}=24.0^{\circ}$, no solution could be obtained for reasons which are given in section 3.1). The angle between the shock-shock and the interface, $\tilde{\chi}$, is small, reaching a maximum value of $\tilde{\chi}=0.25^{\circ}$ at $\omega_{i}=27.6^{\circ}$, which would explain why the shock-shock and the Mach stem are hardly discernible from the interface in figure 13 (plate 3 ) of Abd-elFattah, Henderson and Lozzi's paper. Further, the transmitted shock wave angle at the interface changes from $\omega_{t}=73.6^{\circ}$ at $\omega_{i}=24.0^{\circ}$ (transition), to $\omega_{t}=76.4^{\circ}$ at $\omega_{i}=27.6^{\circ}$, and does not approach the experimentally observed value of $\omega_{t}=93.0^{\circ}$ for large incident wave angles. The reason for this discrepancy is that the solution according to shock dynamics theory is constrained to predict a shock front that is continuous throughout, whereas the experiments show a discontinuous shock front at the interface.

This range of incident wave angles corresponds to the bound precursor range discussed by Abd-el-Fattah, Henderson and Lozzi. Their photographs show that in this range, the transmitted wave is slightly ahead of the incident wave at the interface. Although their results were not conclusive, they noticed, however, that both the incident and transmitted shock fronts had the same, or very nearly the same velocity along the interface, and they inferred that the discontinuity of the shock front at the interface is the result of some non-pseudostationary process at the corner. Perhaps, if the secondary effects of the corner could be eliminated completely, that is, if the back plate were to be aligned 
perfectly with the front plate with no gap between them, then the bound precursor irregular refraction result would indeed reduce to a simple irregular refraction result.

For $\omega_{i}>27.6^{\circ}$, shock dynamics theory does not yield any solution. As discussed in section 3.2, this is because, as the incident wave angle approaches the limit of $\omega_{i}=27.6^{\circ}$, the shock-expansion behind the interface fills up the entire region, and, at the same time, the shock front at the interface becomes normal to the interface, so that no ray tubes cross the interface. This range of incident wave angles corresponds to the free precursor result discussed by Abd-el-Fattah, Henderson and Lozzi, in which the velocity of the precursor (transmitted wave), $V_{t}$, is greater than the velocity of the incident shock, $V_{i}$, along the interface. In their paper, they determine the incident wave angle for the second transition, that is, the transition to the free precursor result, to be $\omega_{i}=28.8^{\circ}$ or $27.4^{\circ}$, depending on whether or not the membrane inertia is considered. These values compare very favourably with our result of $27.6^{\circ}$.

4.1.3. Abd-el-Fattah and Henderson's results, In a subsequent paper, Abd-elFattah and Henderson (1978) studied the refraction of shock waves at a carbon dioxide - methane interface. Their measurements indicated contamination at the interface, and that the methane was only $90 \%$ pure (10\% by volume carbon dioxide), giving a sound speed ratio across the interface of about 1.54 . In their experiments, they used three different incident shock strengths, $M_{1}=1.11 \mathrm{~B}$, 1.336 and 2.243, which they classified as the very weak group, the weak group and the strong group, respectively. In general, for each of the three groups, at small wave angles, the refraction was regular. This was followed by bound precursor irregular refraction for larger wave angles and by various types of free precursor irregular refractions at very large wave angles. These results are qualitatively the same as those discussed in the previous section, except for the 
occurrence of the different kinds of irregular refraction in the free precursor range.

For the very weak group, the agreement between Abd-el-Fattah and Henderson's experimental results and our shock dynamics computations is very good, especially for the regular refraction range, and the limits of their bound precursor range correspond almost exactly to the limits of our irregular refraction range. However, for the weak group and the strong group, good agreement is obtained only in the regular refraction range. For the weak group, we find that the irregular refraction range extends from transition at an interface angle of $53.2^{\circ}$, to an interface angle of $29^{\circ}$, whereas Abd-el-Fattah and Henderson indicate that the bound precursor range extends from transition at an interface angle of about $53^{\circ}$, to an interface angle of about $44^{\circ}$ only. For the strong group, shock dynamics predicts irregular refraction from transition at $\delta_{I}=51.6^{\circ}$ through $\delta_{I}=0^{\circ}$, that is, until the interface lies on the wall. On the other hand, Abd-el-Fattah and Henderson give a bound precursor result in the range $49.7^{\circ}>\delta_{I}>33.4^{\circ}$, and for interface angles smaller than $33.4^{\circ}$, their observations indicate a free precursor result.

Despite the poor agreement between the limits for the ranges of Abd-elFattah and Henderson's bound precursor refraction results and our irregular refraction results, it is interesting to note that their trajectory path angles of the shock wave confluences, $\chi_{1}$ and $\chi_{2}$, show satisfactory agreement with our shock-shock angle. This appears to indicate that the general form of the shock refraction in both the experiment and the theory are the same, and suggests that the various free precursor results observed may have been caused by the experimental set-up.

The reason for these discrepancies is not clear at this time. As Abd-el-Fattah and Henderson have pointed out, their results do not agree entirely with the 
results obtained by Jahn (1956), and in particular, they do not record any cases of irregular refraction in which the transmitted wave is continuous with the Mach stem, as shown in Jahn's figure 14(c). Their experimental results also indicate that for large wave angles, that is, for small interface angles, a corner signal attenuates the reflected wave at the three shock confluence. As mentioned in the previous section, this corner signal may have been caused by the experimental set-up. It appears that further analysis will be required when more experimental data becomes available.

\subsection{Comparison with three-shock theory}

Abd-el-Fattah, Henderson and Lozzi (1976) have computed the transition angles for refraction of a plane shock at a pure carbon dioxide - helium interface as a function of the incident shock strength. They use a method developed by Henderson (1966), based on the behaviour of three shock waves meeting at a point, in which the full equations of motion are applied to the local shock geometry at the interaction point on the interface, and are solved graphically in the hodograph plane using the shock polar method. Their results are shown by the solid line in figure 4.2 , which is reproduced from their paper, where the variable $\eta_{i}$ is

$$
\eta_{i}=\frac{2 \gamma M_{1}^{2}-(\gamma-1)}{\gamma+1}
$$

The results of the present theory have been entered as data points in the figure. It is seen that for Mach numbers below 3 , the agreement is very good. Again, for simplicity, we have taken the ratio $\gamma_{2} / \gamma_{1}$ across the interface to be unity, instead of using the actual value of 1.28 . 


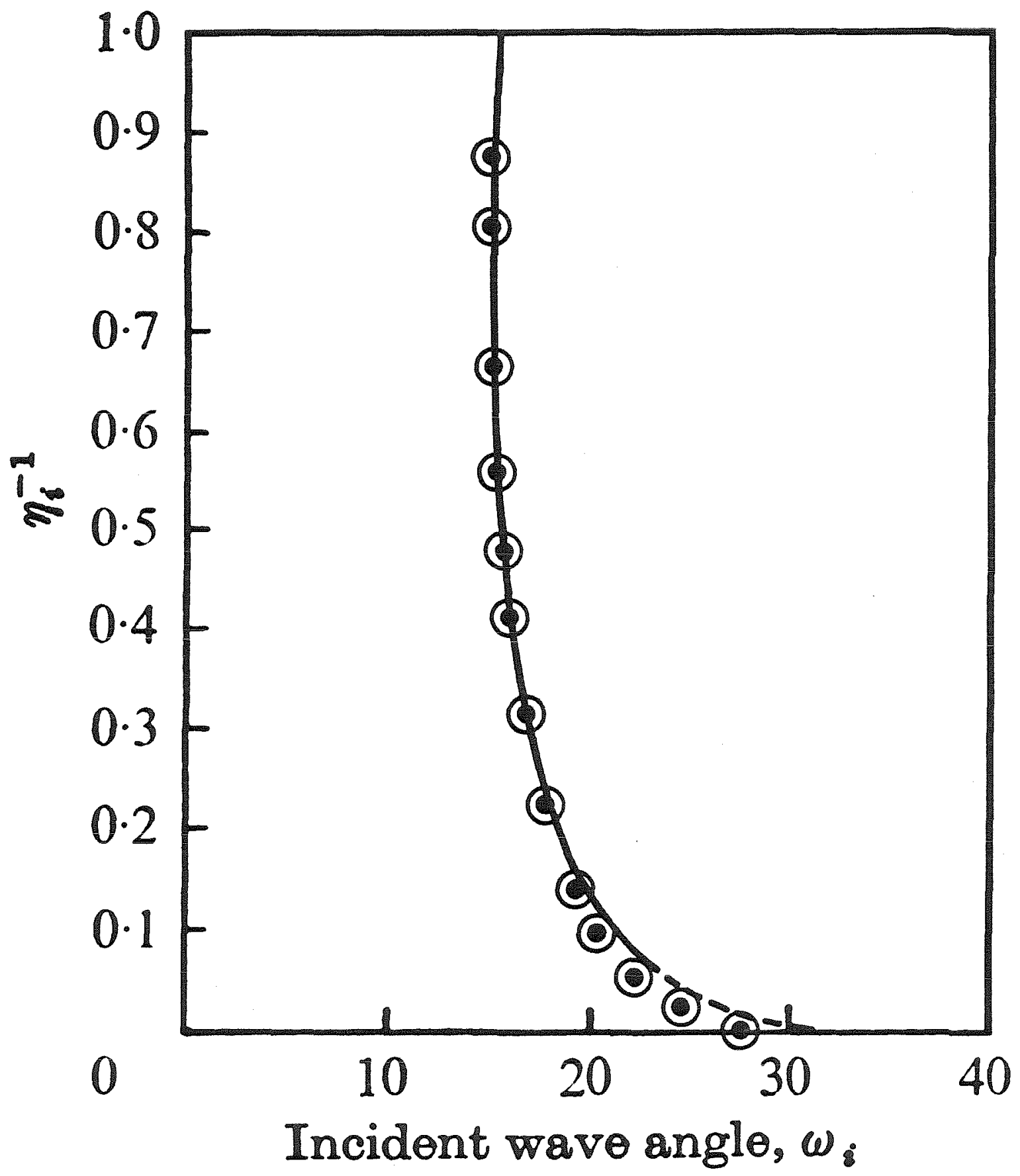

FIGURE 4.2. Transition angles for refraction of a plane shock at a pure carbon dioxide - helium interface. (อ), present theory; _- Abd-ElFattah, Henderson \& Lozzi's results. 


\subsection{Normal interaction}

An interesting check of the accuracy of the theory can be made in the case of Problem 1 with $\delta_{I}=90^{\circ}$, for which the shock interacts normally with the interface (table 4.2). Since the transmitted wave in region 2 is calculated exactly in this analysis from the one-dimensional gasdynamics equations, while the transmitted wave in region 3 is calculated approximately by shock dynamics theory, the departure of $M_{3}$ from $M_{2}$ and of $\theta_{3}$ from 0 provide a measure of the accuracy of the theory. It is seen that the theory shows reasonable agreement, although the accuracy decreases as the ratio $a_{02} / a_{01}$ increases, as might have been expected. The results of a more complete analysis of this case is depicted in figure 4.3 , in which the error $\varepsilon$ is defined by

$$
\varepsilon=\frac{M_{3}-M_{2}}{M_{2}}
$$

Table 4,2. Calculated refraction parameters for normal interaction

\begin{tabular}{c|cc|cccc} 
Case & $M_{1}$ & $a_{02} / \alpha_{01}$ & $M_{2}$ & $\theta_{2}$ & $M_{3}$ & $\theta_{3}$ \\
\hline 47 & 1.5 & 1.5 & 1.39 & 0.0 & 1.40 & 0.0 \\
48 & 1.5 & 5.0 & 1.15 & 0.0 & 1.20 & 0.0 \\
49 & 5.0 & 1.5 & 4.03 & 0.0 & 4.08 & 0.0 \\
50 & 5.0 & 5.0 & 2.09 & 0.0 & 2.42 & 0.0
\end{tabular}

Angles $\theta$ are given in degrees. 


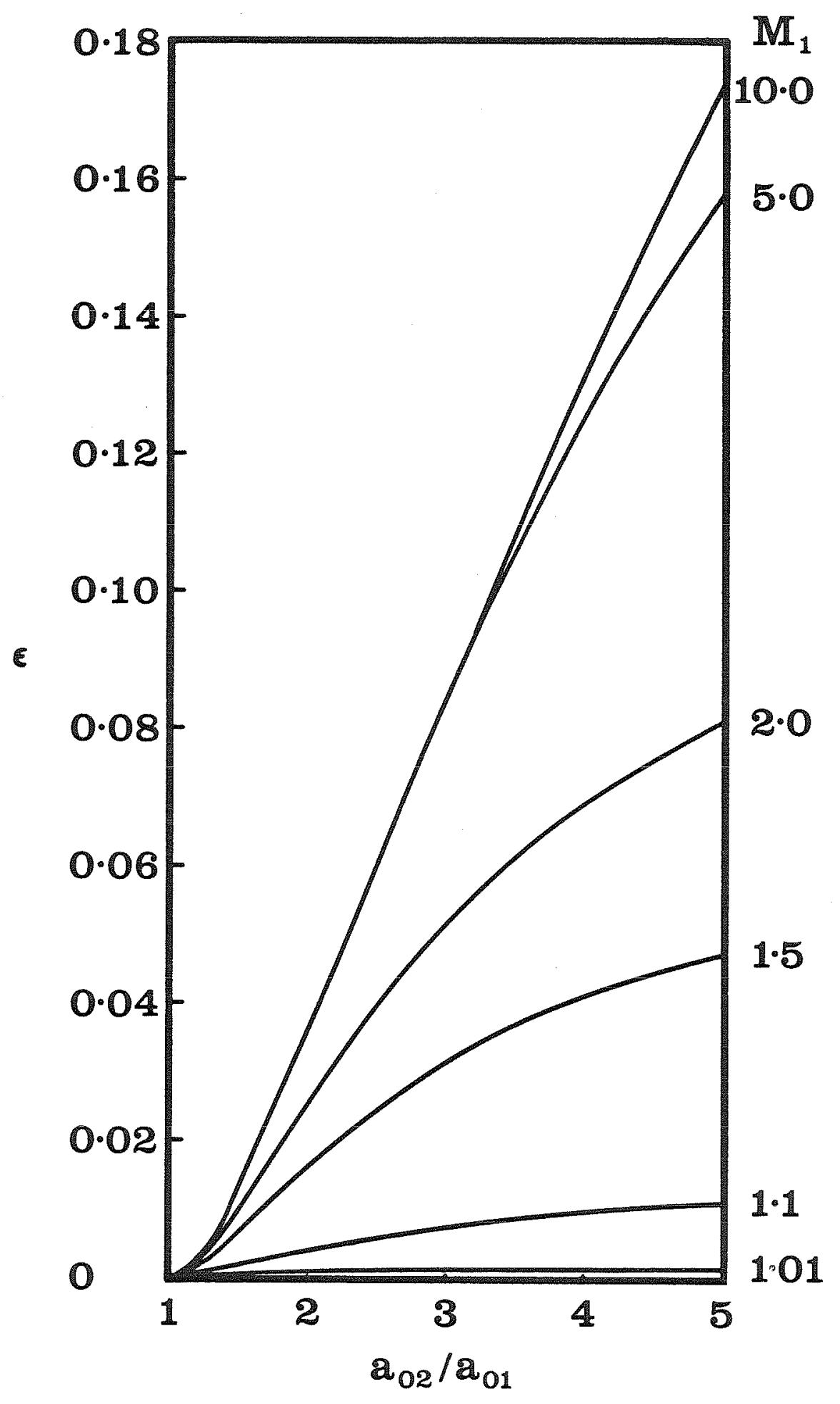

FIGURE 4.3. Relative error between the results from shock dynamics and the results from one-dimensional gasdynamies for normal interaction, $\delta_{I}=90^{\circ}$. 


\subsection{Closing remarks}

In closing, we consider briefly the results of a problem analysed by Collins and Chen (1970a), who applied the theory of shock dynamics to the propagation of a shock in two half-planes separated by a free surface, that is, $\delta_{I}=0^{\circ}$, with $M_{1}=4.45$ and $a_{02} / a_{01}=1.41$. This problem is qualitatively similar to our case 12 .

As discussed in Chapter 1, their analysis does not properly account for the transmission and reflection of both families of characteristics at the interface, and further, it does not provide for the possible occurrence of shock-shocks. Their solution essentially has two regions that lie adjacent to the interface, and they find that the ray angle changes from about $19^{\circ}$ above the interface to about $3^{\circ}$ below. On the other hand, our solution contains two shock-shocks, one at $27.2^{\circ}$ and the other at $1.0^{\circ}$ above the interface, and it has two uniform regions, together with a shock-expansion which lies adjacent to and below the interface. The configuration is similar to that shown in figure 3.3(e). At the interface, we find that the ray angle changes from $45.5^{\circ}$ above to $23.2^{\circ}$ below. 


\section{Chapter 5}

\section{SUMMARY AND CONCLUSIONS}

Whitham's theory of shock dynamics has been reformulated to account for imposed non-uniformities in the undisturbed medium ahead of the shock front. The governing equations are hyperbolic in nature. When compared to the conventional shock dynamics equations for a uniform medium, the essential difference is the appearance of source terms, which represent disturbances generated on the shock front as the shock propagates into regions where the fluid properties are non-uniform.

As in the case of a uniform medium, discontinuities of shock strength, $M$, and slope, $\theta$, can occur in the form of shock-shocks, which are the manifestation of the triple-shock intersections in Mach reflection. However, the presence of a contact surface, across which there exists an imposed change in fluid properties, introduces another kind of discontinuity in the shock front. Jump conditions across the interface are developed from the characteristic equations, and the theory naturally provides a relationship analogous to Snell's law. The shock dynamics equations governing shock refraction at a gaseous interface are simple to apply and yield a unique solution. By changing various parameters such as the incident shock strength, the fluid property ratios across the interface, and the interface angle, a large variety of interesting configurations are easily obtained. These features give the present theory its greatest appeal over existing methods for calculating shock refraction.

The theory models both regular refraction and irregular refraction, and also predicts the transition from one to the other. This is in contrast to the diffraction of shock waves in a uniform medium over solid wedges, where the 
theory only models irregular refraction. Transition from regular to irregular refraction in shock dynamics theory is analogous to the transition which occurs in unsteady one-dimensional gasdynamics under the action of a moving force field or "leaky piston", in which the solution changes from one that is shock-free for a supersonic force, to one that contains a shock for a subsonic force. In both problems, a sonic configuration occurs that is analogous to ChapmanJouget detonation.

For irregular refraction, given an incident shock strength and fluid property ratio, there exists an interíace angle at which the Mach stem is normal to the interface, so that no rays cross the interface. This is the condition for no energy flow across the interface, and it is the same condition that is applied at a solid boundary in the analysis of shock diffraction using the theory of shock dynamies in a uniform medium.

in the neighbourhood of a solid boundary, under certain conditions, a limiting irregular case is obtained, in which the Mach stem becomes normal to the interface and the Mach number of the shock front at the wall tends to unity. It appears that this signals the onset of a discontinuous shock front at the interface, namely, the formation of a precursor wave.

It has been implicitly assumed, through the application of the characteristic rule, that modifying disturbances overtaking the main shock front are excluded. Further, as pointed out by Whitham (1957), the theory tends to overconcentrate the disturbances on the shock front. For strong shocks, this representation is satisfactory, but for weak shocks, the true disturbance is distributed over a larger region than predicted by the theory. In spite of these factors, it appears that the calculated shock parameters compare favourably with the limited experimental data available. In particular, the calculated wave angles for the transmitted shock in regular refraction show very good agreement with 
experiment, while the calculated angles for transition from regular to irregular refraction agree well with the predictions from three-shock theory.

Future work will be directed at investigating problems involving the fast-slow gaseous interface, and also at examining shock refraction problems in which the variation of fuid properties is continuous. 


\section{References}

ABD-EL-FATTAH, A.M. \& HENDERSON, L.F. 1978 Shock waves at a slow-fast gas interface. J. Fluid Mech., 89, 79-95.

ABD-EL-FATTAH, A.M., HENDERSON, L.F. \& LOZZI, A. 1976 Precursor shock waves at a slow-fast gas interface. J. Fluid Mech., 76, 157-76.

BRYSON, A.E. \& GROSS, R.W.F. 1961 Diffraction of strong shock waves by cones, cylinders and spheres. J. Fluid Mech., 10, 1-22.

CHESTER, W. 1954 The quasi-cylindrical shock tube. Phil. Mag., (7) 45, 1293-301. CHISNELL, R.F. 1955 The normal motion of a shock wave through a nonuniform one-dimensional medium. Proc. Roy. Soc., A 232, 350-70.

CHISNELL, R.F. 1957 The motion of a shock wave in a channel, with applications to cylindrical and spherical shock waves. J. Fluid Mech, 2, 286-98.

COLLINS, R. \& CHEN, H.T. 1970 Propagation of a shock wave of arbitrary strength in two half planes containing a free surface. J. Comp. Phys., 5, 415-22.

COLLINS, R. \& CHEN, H.T, 1970 Motion of a shock wave through a nonuniform fluid. Lecture Notes in Physics, 8, 264-9. Springer.

HAYES. W.D. 1968 The propagation upward of the shock wave from a strong explosion in the atmosphere. I. Fluid Mech., 32, 317-31.

HENDERSON, L.F. 1966 The refraction of a plane shock wave at a gas interface. J. Fluid Mech., 26, 607-37.

HENDERSON, L.F. 1980 On the Whitham theory of shock-wave diffraction at concave corners. J. Fluid Mech, 99, B01-11.

HOFFMAN, A.L. 1967 A single-fluid model for shock formation in MHD shock tubes. J. Plasma Physics, 1, 193-207.

JAHN, R.G. 1956 The refraction of shock waves at a gaseous interface. J. Fuid Mech, 1, 457-89.

KUTLER, P. \& SHANKAR, V. 1977 Diffraction of a shock wave by a compression corner: Part II - single Mach reflection. A.I.A.A. J, 15, 197-203.

POLACHEK, H. \& SEEGER, R.J. 1951 On shock-wave phenomena; refraction of shock waves at a gaseous interface. Phys. Rev., B4, 922-29.

SHANKAR, V., KUTLER, P. \& ANDERSON, D. 1978 Diffraction of a shock wave by a compression corner: Part I - regular refraction. A.I.A.A. J., 16, 4-5.

TAUB, A.H. 1947 Refraction of plane shock waves. Phys, Rev., 72, 51-60.

WHITHAM, G.B. 1957 A new approach to problems of shock dynamics. Part I. Two-dimensional problems. J. Fluid Mech., 2, 145-71.

WHITHAM, G.B. 1958 On the propagation of shock waves through regions of nonuniform area or flow. J. Fluid Mech, 4, 337-60.

WHITHAM, G.B. 1959 A new approach to problems of shock dynamies. Part II. Three-dimensional problems. J. Fluid Mech., 5, 369-86. 


\section{Appendix A}

\section{INTEGRATION OF THE RAY TUBF ARFA FUNCTTON}

From equation (2.1.26), which is valid only in a uniform region, we have

$$
\frac{d A}{A}+\frac{M \lambda(M)}{M^{2}-1} d M=0
$$

where

$$
\lambda(M, \gamma)=\left(1+\frac{2}{\gamma+1} \frac{1-\mu^{2}}{\mu}\right)\left(1+2 \mu+\frac{1}{M^{2}}\right)
$$

and

$$
\mu(M, \gamma)=\left[\frac{(\gamma-1) M^{2}+2}{2 \gamma M^{2}-(\gamma-1)}\right]^{1 / 2}
$$

On integrating (A.1), we obtain

$$
A=C \exp ^{-\zeta(M)} \text {, }
$$

where

$$
\xi(M)=\int \frac{M \lambda(M)}{M^{2}-1} d M
$$

and $C$ is an arbitrary constant. From (A.5), using (A.R) 


$$
\begin{aligned}
\xi(M)=\int\left\{\frac{M}{M^{2}-1}\left(1+\frac{1}{M}\right)+\frac{2 \mu M}{M^{2}-1}+\frac{2}{\gamma+1} \frac{M\left(1-\mu^{2}\right)}{\mu\left(M^{2}-1\right)}+\right. \\
\left.+\frac{4}{\gamma+1} \frac{M\left(1-\mu^{2}\right)}{\left(M^{2}-1\right)}+\frac{2}{\gamma+1} \frac{\left(1-\mu^{2}\right)}{\mu M\left(M^{2}-1\right)}\right\} d M
\end{aligned}
$$

which on integration yields

$$
\begin{aligned}
& \zeta(M)=\log \left(\frac{M^{2}-1}{M}\right)+\left[\log \left(\frac{1-\mu}{1+\mu}\right)+\left(\frac{(\gamma-1)}{2 \gamma}\right)^{1 / 2} \log \left\{\frac{\mu+\left(\frac{\gamma-1}{2 \gamma}\right)^{1 / 2}}{\mu-\left(\frac{\gamma-1}{2 \gamma}\right)^{1 / 2}}\right]+\right. \\
& +\left(\frac{2}{\gamma(\gamma-1)}\right)^{1 / 2} \log \left[\left(M^{2}+\frac{2}{\gamma-1}\right)^{1 / 2}+\left(M^{2}-\frac{\gamma-1}{2 \gamma}\right)^{1 / 2}\right]+ \\
& +\frac{1}{\gamma} \log \left(M^{2}-\frac{\gamma-1}{2 \gamma}\right)-\left(\frac{2}{\gamma-1}\right)^{1 / 2} \tan ^{-1}\left[\mu\left(\frac{\gamma-1}{2}\right)^{1 / 2}\right] \\
& =\log \left(\frac{M^{2}-1}{M}\right)+\log \left(\frac{1-\mu}{1+\mu}\right)+\left(\frac{\gamma}{2(\gamma-1)}\right)^{1 / 2} \log \left\{\frac{\mu+\left(\frac{\gamma-1}{2 \gamma}\right)^{1 / 2}}{\mu-\left(\frac{\gamma-1}{2 \gamma}\right)^{1 / 2}}\right\}+ \\
& +\frac{1}{\gamma} \log \left(M^{2}-\frac{\gamma-1}{2 \gamma}\right)-\left(\frac{2}{\gamma-1}\right)^{1 / 2} \tan ^{-1}\left[\mu\left(\frac{y-1}{2}\right)^{1 / 2}\right]+ \\
& +\left[\frac{1}{2 \gamma(\gamma-1)}\right]^{1 / 2} \log \left[\frac{4 \gamma-(\gamma-1)^{2}}{4 \gamma^{2}}\right] .
\end{aligned}
$$

The last term of (A.7) is a constant, so that from (A.4) 


$$
\begin{gathered}
A=C\left(\frac{M}{M^{2}-1}\right)\left(\frac{1+\mu}{1-\mu}\right)\left(M^{2}-\frac{\gamma-1}{2 \gamma}\right)^{-1 / \gamma}\left[\frac{\mu-\left(\frac{\gamma-1}{2 \gamma}\right)^{1 / 2}}{\mu+\left(\frac{\gamma-1}{2 \gamma}\right)^{1 / 2}}\right]^{\left.\frac{\gamma}{2(\gamma-1)}\right]^{1 / 2}} \times \\
\quad \times \exp \left[\left(\frac{2}{\gamma-1}\right)^{1 / 2} \tan ^{-1}\left\{\mu\left(\frac{\gamma-1}{2}\right)^{1 / 2}\right\}\right]
\end{gathered}
$$

where $C$ is an arbitrary constant.

Equation (A.B) is a more compact form of the expression obtained independently by Henderson (1980), who also pointed out the errors in the expression published by Bryson and Gross (1961). 


\section{Appendix B}

\section{THE COORDINATE TRANSTORMATION REILATIONS}

From figure B.1, by considering a line element $A \delta \beta$ on the shock front ( $\alpha=$ constant), we get

$$
\delta x=-A \delta \beta \sin \theta
$$

and

$$
\delta y=A \delta \beta \cos \theta
$$

that is,

$$
\left(\frac{\partial x}{\partial \beta}\right)_{\alpha}=-A \sin \theta
$$

and

$$
\left(\frac{\partial y}{\partial \beta}\right)_{\alpha}=A \cos \theta
$$

Similarly, by considering a line element $a_{0} M \delta \alpha$ on the ray ( $\beta=$ constant), we get

$$
\delta x=a_{0} M \delta \alpha \cos \theta
$$

and

$$
\delta y=a_{0} M \delta \alpha \sin \theta .
$$

that is, 


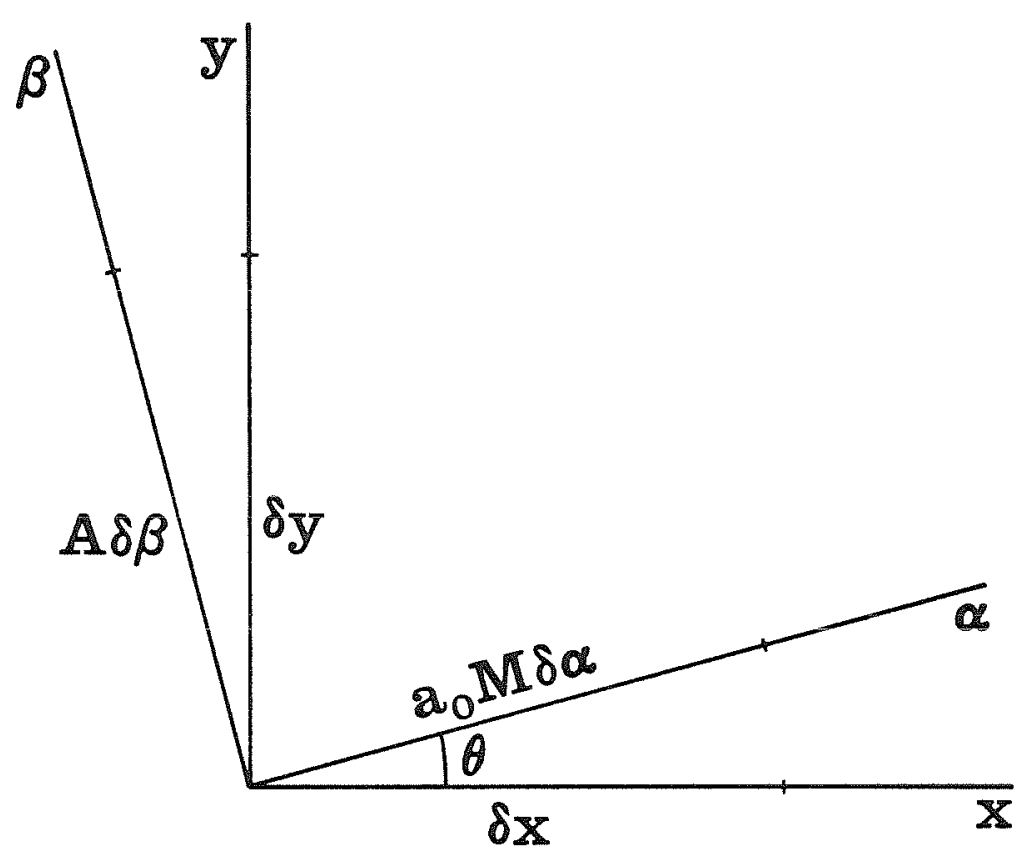

FIGURE B.1. Relationship between the $(\alpha, \beta)$ and the $(x, y)$ coordinate systems. 


$$
\left(\frac{\partial x}{\partial \alpha}\right)_{\beta}=\alpha_{0} M \cos \theta
$$

and

$$
\left(\frac{\partial y}{\partial \alpha}\right)_{\beta}=\mathbb{a}_{0} M \sin \theta .
$$

Likewise, by considering line elements along the $x$-axis and along the $y$-axis, we get

$$
\begin{aligned}
& \left(\frac{\partial \alpha}{\partial y}\right)_{x}=\frac{\sin \theta}{a_{0} M}, \\
& {\left[\frac{\partial \beta}{\partial y}\right]_{x}=\frac{\cos \theta}{A} .}
\end{aligned}
$$

and

$$
\begin{aligned}
& \left(\frac{\partial \alpha}{\partial x}\right)_{y}=\frac{\cos \theta}{\alpha_{0} M} . \\
& \left(\frac{\partial \beta}{\partial x}\right)_{y}=-\frac{\sin \theta}{A} .
\end{aligned}
$$

Using (B.1) through (B.4), we obtain the total differential relations

$$
\begin{aligned}
& d x=a_{0} M \cos \theta d \alpha-A \sin \theta d \beta, \\
& d y=a_{0} M \sin \theta d \alpha+A \cos \theta d \beta,
\end{aligned}
$$




$$
\begin{aligned}
& d \alpha=\frac{\cos \theta}{a_{0} M} d x+\frac{\sin \theta}{a_{0} M} d y, \\
& d \beta=-\frac{\sin \theta}{A} d x+\frac{\cos \theta}{A} d y .
\end{aligned}
$$

and the derivatives

$$
\begin{aligned}
& \frac{\partial}{\partial x}=\frac{\cos \theta}{a_{0} M} \frac{\partial}{\partial \alpha}-\frac{\sin \theta}{A} \frac{\partial}{\partial \beta}, \\
& \frac{\partial}{\partial y}=\frac{\sin \theta}{a_{0} M} \frac{\partial}{\partial \alpha}+\frac{\cos \theta}{A} \frac{\partial}{\partial \beta}, \\
& \frac{\partial}{\partial \alpha}=a_{0} M \cos \theta \frac{\partial}{\partial x}+a_{0} M \sin \theta \frac{\partial}{\partial y} . \\
& \frac{\partial}{\partial \beta}=-A \sin \theta \frac{\partial}{\partial x}+A \cos \theta \frac{\partial}{\partial y} .
\end{aligned}
$$

These are the transformation relations between the $(\alpha, \beta)$ coordinate system and the $(x, y)$ coordinate system. 


\section{Appendix C}

\section{THE SINGULARITY}

In this appendix, we examine the conditions under which the ordinary differential equation (2.4.10) becomes singular and prove that the singularity is integrable.

\section{c.1. Manifestation of the singularity}

From equation (2.4.10), using (2.4.11) and (2.4.12), we have the differential equation

$$
\frac{d M}{d \alpha_{0}}=\frac{\nu^{2} M\left[(1+g) \alpha_{0}^{2} M^{2}-g \alpha_{01}^{2} C_{1}^{2}\right]}{\alpha_{0}\left[\alpha_{01}^{2} C_{1}^{2}-\left(1+\nu^{2}\right) \alpha_{0}^{2} M^{2}\right]}
$$

where

$$
C_{1}=\frac{M_{1}}{\cos \widetilde{\theta}_{1}}
$$

and $g$ and $\nu$ are functions of $M$, given by equations (2.1.23) and (2.3.15) respectively. We note that $C_{1}$ is constant for a given problem. It is convenient to non-dimensionalize the terms in $\alpha_{0}$ by writing

$$
r=\frac{a_{0}}{a_{01}}
$$

to give 


$$
\frac{d M}{d r}=\frac{\nu^{2} M\left[(1+g) r^{2} M^{2}-g C_{1}^{2}\right]}{r\left[C_{1}^{2}-\left(1+\nu^{2}\right) r^{2} M^{2}\right]}
$$

The differential equation becomes singular when the denominator vanishes, that is,

$$
C_{1}^{2}-\left(1+\nu^{2}\right) r^{2} M^{2}=0
$$

From (2.4.8), we have

$$
\frac{a_{0} M}{\cos \tilde{\theta}}=\frac{a_{01} M_{1}}{\cos \tilde{\theta}_{1}}
$$

that is,

$$
\frac{r M}{\cos \widetilde{\theta}}=C_{1}
$$

So (C.1.5) becomes

$$
C_{1}^{2}-\left(1+\nu^{2}\right) C_{1}^{2} \cos ^{2} \ddot{\theta}=0
$$

that is,

$$
\cos ^{2} \tilde{\theta}=\frac{1}{1+\nu^{2}}
$$




$$
\tan \widetilde{\theta}= \pm \nu .
$$

Applying (2.3.19), we get

$$
\widetilde{\theta}= \pm v \text {. }
$$

that is, the singularity occurs when

$$
\theta=\delta_{I} \pm v
$$

The characteristics slopes, from (2.3.20), are

$$
\eta^{ \pm}=\theta \pm v
$$

so that when the singularity occurs,

$$
\eta^{+}=\delta_{I} \quad \text { or } \quad \delta_{I}+2 v
$$

or

$$
\eta^{-}=\delta_{I} \quad \text { or } \quad \delta_{I}-2 v
$$

Thus, one condition for the differential equation (2.4.10) to be singular is that a characteristic lies parallel to the interface. We note that this condition also causes the source terms in equations (2.4.5) to be singular. 


\section{C.2. Behaviour of the differential equation at the singularity}

To study the behaviour of (C.1.1) at the singularity, it is first shown that $M$ is bounded, and then the right-hand side of the equation is expanded in a Taylor series about the singular point $\left(r_{0}, M_{0}\right)$.

From equation (C.1.5), at the singularity, we have

$$
M^{2}=\frac{C_{1}^{2}}{r^{2}\left[1+\nu^{2}(M)\right]} .
$$

From (C.1.2), the bounds on the constant $C_{1}$ are

$$
\left|C_{1}\right|<\infty \text { for } \tilde{\theta} \neq \frac{\pi}{2} \text {. }
$$

for finite sound speed ratios,

$$
0<r<\infty \text {, }
$$

and from (2.3.15), or from figure 2.2, as $M$ varies from $M=1$ to $M \gg 1$, we have

$$
0 \leq \nu(M)<\left[\frac{1}{1+\frac{2}{\gamma}+\left(\frac{2 \gamma}{\gamma-1}\right)^{1 / 2}}\right]^{1 / 2} \approx 0.444
$$

Therefore, from (C.R.1) 


$$
M^{2}<\infty
$$

that is, $M$ is bounded.

Since $M$ is bounded, we can take a Taylor series expansion about the singular point $\left(r_{0}, M_{0}\right)$. We write (C.1.4) as

$$
\frac{d M}{d r}=\frac{\Psi(M)}{r\left[C_{1}^{2}-r^{2} \psi^{2}(M)\right]} .
$$

where

$$
\Psi(\bar{M})=\frac{\nu^{2} M\left(1-\nu^{2} g\right) C_{1}^{2}}{1+\nu^{2}}
$$

and

$$
\psi(M)=M^{2}\left(1+\nu^{2}\right)
$$

Taking the Taylor series expansion gives

$$
\frac{d M}{d r}=\frac{\Psi\left(M_{0}\right)}{\left[r_{0}+\left(r-r_{0}\right)+\ldots\right]\left[C_{1}^{2}-\left\{r_{0}+\left(r-r_{0}\right)+\ldots\right\}^{2}\left\{\psi\left(M_{0}\right)+\left(M-M_{0}\right) \psi\left(M_{0}\right)+\ldots\right\}^{2}\right]}
$$

which, to first order is

$$
\frac{d M}{d r}=-\frac{\Psi\left(M_{0}\right)}{2 r_{0}^{2}\left[\left(r-r_{0}\right) \psi^{2}\left(M_{0}\right)+r_{0}\left(M-M_{0}\right) \psi\left(M_{0}\right) \psi^{\prime}\left(M_{0}\right)\right]}
$$


The terms in $r$ and $M$ are not separable in this form, so further linearization is necessary. For this, we assume that in the neighbourhood of the singularity

$$
\frac{\left|r-r_{0}\right|}{\left|M-M_{0}\right|} \ll 1
$$

Then (C.2.3) becomes

$$
\frac{d M}{d r}=-\frac{\Phi_{0}^{2}}{2\left(M-M_{0}\right)}
$$

where

$$
\Phi_{0}^{Z}=\frac{\Psi\left(M_{0}\right)}{r_{0}^{3} \psi\left(M_{0}\right) \psi\left(M_{0}\right)} .
$$

Integration of (C.2.5) gives

$$
\left(M-M_{0}\right)^{2}=-\Phi_{0}^{2} r+C
$$

where $C$ is a constant. At the singularity, $r=r_{0}$ and $M=M_{0}$, so

$$
c=\Phi_{0}^{2} r_{0}^{2}
$$

giving

$$
M=M_{0} \pm \Phi_{0}\left(r_{0}-r\right)^{1 / 2}
$$

Hence, to this first order approximation, 


$$
\frac{d M}{d r} \sim \pm \frac{1}{\left(r_{0}-r\right)^{1 / 2}}
$$

that is, the singularity is integrable.

We now return to check assumption (C.2.4). Substituting for $M-M_{0}$ from (C.2.6) gives, in the neighbourhood of the singularity,

$$
\left|\left(r_{0}-r\right)^{1 / 2}\right| \ll 1
$$

that is, the assumption is consistent, and the proof is complete. 


\section{Appendix D}

\section{SHOCK WAVE INTTRACTION WTTH A NORMAL CONTACT DISCONTINUITY}

When a shock wave is incident normally on an interface separating two regions of gas with different sound speeds, in general, the result is a transmitted wave and a reflected wave. The transmitted wave is always a shock, while the reflected wave may be either a shock or an expansion, depending on the properties of the gas on each side of the interface. The two cases are illustrated in figures D.1(a) and D.1(b) respectively.

The strengths of the transmitted and reflected waves can be found by matching the pressures and gas velocities across the interface. In this analysis, it is assumed that only one species of gas is present, that is, we take $\gamma=$ constant across the interface. Also, we assume the perfect gas relations.

\section{D.1. Refected shock wave}

From figure D.1(a) and the shock relations given in equation (2.1.16), across the incident shock

$$
\frac{u_{11}}{\alpha_{01}}=\frac{2\left(M_{i}^{2}-1\right)}{(\gamma+1) M_{i}}
$$

and

$$
\frac{p_{11}}{p_{01}}=1+\frac{2 \gamma\left(M_{i}^{2}-1\right)}{\gamma+1}
$$

across the transmitted shock 


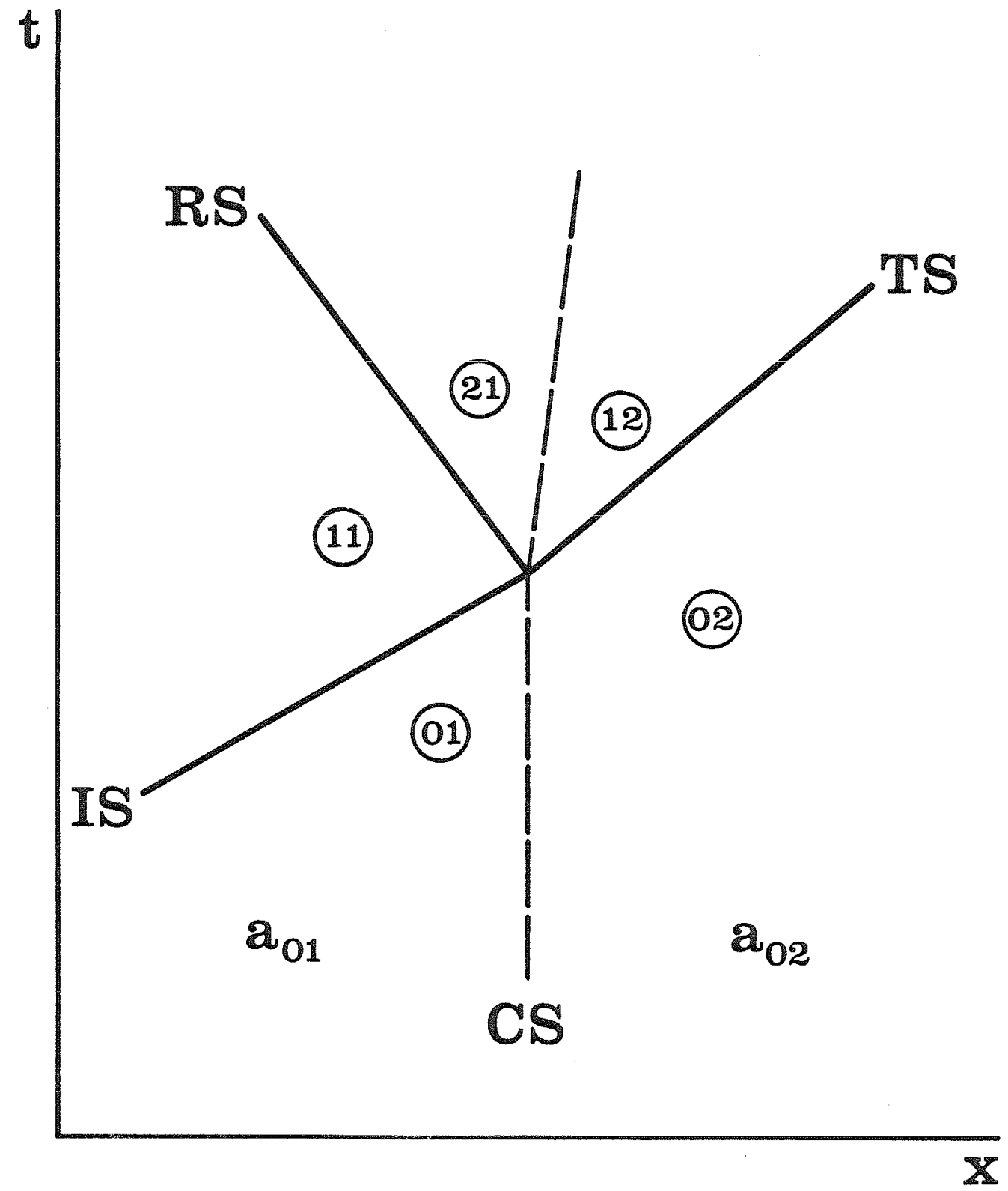

FIGURE D.1(a). Interaction of a shock wave with a normal contact discontinuity when the reflected wave is a shock. CS, contact surface; IS, incident shock; TS, transmitted shock; RS, reflected shock. 


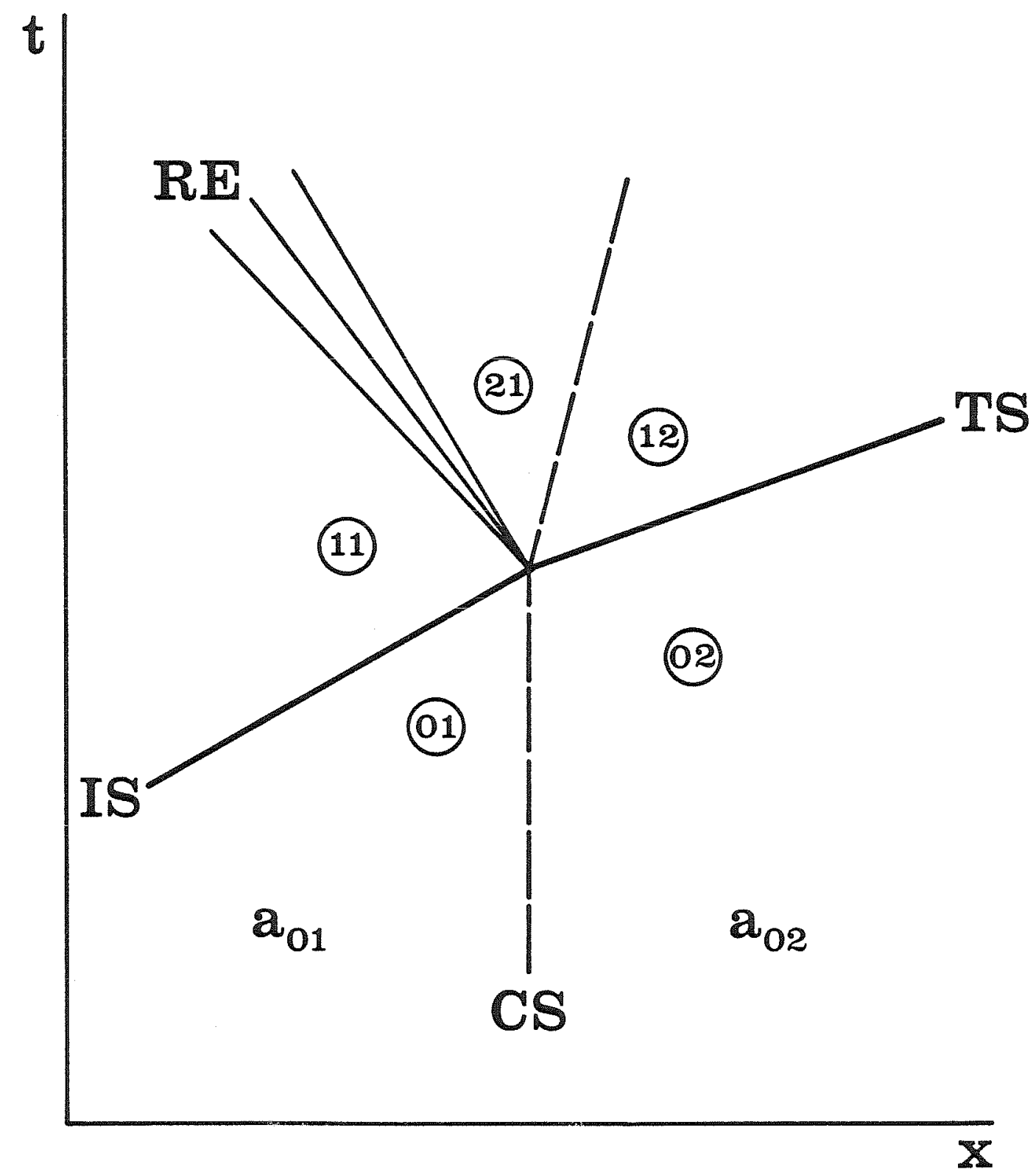

FIGURE D.1(b). Interaction of a shock wave with a normal contact discontinuity when the reflected wave is an expansion. CS, contact surface; IS, incident shock; TS, transmitted shock; RE, reflected expansion. 


$$
\frac{u_{12}}{a_{02}}=\frac{2\left(M_{t}^{2}-1\right)}{(\gamma+1) M_{t}}
$$

and

$$
\frac{p_{12}}{p_{02}}=1+\frac{Z \gamma\left(M_{t}^{2}-1\right)}{\gamma+1} .
$$

and across the reflected shock

$$
\frac{-u_{21}-\left(-u_{11}\right)}{a_{11}}=\frac{2\left(M_{r}^{2}-1\right)}{(\gamma+1) M_{r}}
$$

and

$$
\frac{p_{21}}{p_{11}}=1+\frac{2 \gamma\left(M_{r}^{2}-1\right)}{\gamma+1} .
$$

Also, the sound speed ratio across the incident shock is given by

$$
\frac{a_{11}}{a_{01}}=\frac{\left[\left\{(\gamma-1) M_{i}^{2}+2\right\}\left\{2 \gamma M_{i}^{2}-(\gamma-1)\right\}\right]^{1 / 2}}{(\gamma+1) M_{i}} .
$$

Finally, from the initial conditions

$$
p_{01}=p_{02}
$$

and from the matching conditions 


$$
p_{21}=p_{12}
$$

and

$$
u_{21}=u_{12}
$$

From equations $(D .1 .2),(D .1 .4),(D .1 .6),(D .1 .8)$ and $(D .1 .9)$ we get

$$
M_{\tau}=\left[1+\frac{(\gamma+1)\left(M_{t}^{2}-M_{i}^{2}\right)}{2 \gamma M_{i}^{2}-(\gamma-1)}\right]^{1 / 2} \text {. }
$$

and from equations $(D .1 .1),(D .1 .3),(D .1 .5)$ and $(D .1 .10)$, we get

$$
\left(\frac{M_{i}^{2}-1}{M_{i}}-\frac{M_{t}^{2}-1}{M_{t}} \frac{a_{02}}{a_{01}}\right) \frac{a_{01}}{a_{11}}=\frac{M_{\tau}^{2}-1}{M_{T}}
$$

Eliminating $a_{01} / a_{11}$ using equation (D.1.7) and $M_{r}$ using equation (D.1.11) gives

$$
\begin{aligned}
& {\left[\frac{M_{i}^{2}-1}{M_{i}}-\frac{M_{i}^{2}-1}{M_{t}} \frac{\alpha_{02}}{\alpha_{01}}\right] \frac{M_{i}}{\left[(\gamma-1) M_{i}^{2}+2\right]^{1 / 2}}+} \\
& -\frac{\left(M_{i}^{2}-M_{i}^{2}\right)}{\left[2 \gamma M_{i}^{2}-(\gamma-1)+(\gamma+1)\left(M_{i}^{2}-M_{i}^{2}\right)\right]^{1 / 2}}=0 .
\end{aligned}
$$

Equation (D.1.13) gives the strength of the transmitted shock at a normal interface in terms of the incident shock strength and the sound speed ratio when the reflected wave is a shock. 


\section{D.2. Refected expansion wave}

From figure D.1(b), as in section D.1, across the incident shock

$$
\frac{u_{11}}{a_{01}}=\frac{2\left(M_{i}^{2}-1\right)}{(\gamma+1) M_{i}}
$$

and

$$
\frac{p_{11}}{p_{01}}=1+\frac{2 \gamma\left(H_{i}^{2}-1\right)}{\gamma+1} \text {. }
$$

and across the transmitted shock

$$
\frac{u_{12}}{a_{02}}=\frac{2\left(M_{t}^{2}-1\right)}{(\gamma+1) M_{t}}
$$

and

$$
\frac{p_{12}}{p_{02}}=1+\frac{2 \gamma\left(M_{t}^{2}-1\right)}{\gamma+1} .
$$

Across the reflected expansion wave, the Riemann invariant on the $C^{+}$characteristic is conserved, so that

$$
u_{11}+\frac{2}{\gamma-1} a_{11}=u_{21}+\frac{2}{\gamma-1} a_{21} .
$$

and, since the expansion process is isentropic 


$$
\frac{p_{21}}{p_{11}}=\left(\frac{T_{21}}{T_{11}}\right)^{\frac{\gamma}{\gamma-1}}=\left(\frac{a_{21}}{a_{11}}\right)^{\frac{2 \gamma}{\gamma-1}} .
$$

Also, the sound speed ratio across the incident shock is given by

$$
\frac{a_{11}}{a_{01}}=\frac{\left[\left\{(\gamma-1) M_{i}^{2}+2\right\}\left\{2 \gamma M_{i}^{2}-(\gamma-1)\right\}\right]^{1 / 2}}{(\gamma+1) M_{i}}
$$

Finally, from the initial conditions

$$
p_{01}=p_{02}
$$

and from the matching conditions

$$
p_{21}=p_{12}
$$

and

$$
u_{21}=u_{12}
$$

From equations (D.2.2), (D.2.4), (D.2.6), (D.2.8) and (D.2.9) we get

$$
\frac{a_{21}}{a_{11}}=\left[\frac{2 \gamma M_{t}^{2}-(\gamma-1)}{2 \gamma M_{i}^{2}-(\gamma-1)}\right]^{\frac{\gamma-1}{2 \gamma}}
$$

and from equations (D.2.5) and (D.2.10) we get 


$$
\frac{1}{\gamma+1}\left(\frac{M_{i}^{2}-1}{M_{i}}-\frac{M_{t}^{2}-1}{M_{t}} \frac{a_{02}}{a_{01}}\right) \frac{a_{01}}{a_{11}}=\frac{1}{\gamma-1}\left(\frac{a_{21}}{a_{11}}-1\right) .
$$

Eliminating $\alpha_{01} / \alpha_{11}$ using equation (D.2.7) and $\alpha_{21} / \alpha_{11}$ using equation (D.2.11) gives

$$
\begin{aligned}
{\left[\frac{M_{i}^{2}-1}{M_{i}}-\frac{M_{t}^{2}-1}{M_{t}} \frac{a_{02}}{a_{01}}\right] } & \frac{M_{i}}{\left[\left\{(\gamma-1) M_{i}^{2}+2\right\}\left\{2 \gamma M_{i}^{2}-(\gamma-1)\right\}\right]^{1 / 2}}+ \\
& +\frac{1}{\gamma-1}\left[1-\left\{\frac{2 \gamma M_{t}^{2}-(\gamma-1)}{2 \gamma M_{i}^{2}-(\gamma-1)}\right\}^{\frac{\gamma-1}{2 \gamma}}\right]=0 .
\end{aligned}
$$

Equation (D.2.13) gives the strength of the transmitted shock at a normal interface in terms of the incident shock strength and the sound speed ratio when the reflected wave is an expansion.

\section{D.3. Some general remarks}

Equation (D.1.13) is an expression for the strength of the transmitted shock when the reflected wave is a shock, while equation (D.2.13) is a similar expression for the case when the refiected wave is an expansion. At the cross-over from a reflected shock to a reflected expansion, the reflected wave is an acoustic wave, so that from equations (D.1.11) or (D.2.11), $M_{t}=M_{i}$. Further, from both equations (D.1.13) and (D.2.13), we see that this occurs when $a_{01}=a_{02}$, that is, the interface vanishes.

The pressure ratio across the reflected wave is 


$$
\begin{aligned}
\frac{p_{21}}{p_{11}} & =\frac{p_{12}}{p_{02}} \frac{p_{01}}{p_{11}} \\
& =\frac{2 \gamma M_{i}^{2}-(\gamma-1)}{2 \gamma M_{i}^{2}-(\gamma-1)} .
\end{aligned}
$$

while the velocity ratio is

$$
\begin{aligned}
\frac{u_{21}}{u_{11}} & =\frac{u_{21}}{a_{02}} \frac{a_{01}}{u_{11}} \frac{a_{02}}{a_{01}} \\
& =\left(\frac{M_{t}^{2}-1}{M_{t}}\right)\left(\frac{M_{i}}{M_{i}^{2}-1}\right) \frac{a_{02}}{a_{01}},
\end{aligned}
$$

that is,

$$
\frac{a_{02}}{a_{01}}=\frac{u_{21}}{u_{11}}\left(\frac{M_{i}^{2}-1}{M_{i}}\right)\left(\frac{M_{t}}{M_{t}^{2}-1}\right)
$$

When the reflected wave is a shock

$$
\frac{p_{21}}{p_{11}}>1
$$

and, remembering that the shock is left-facing, while the flow is to the right

$$
\frac{u_{21}}{u_{11}}<1 .
$$


So from $(D .3 .1)$

$$
M_{t}>M_{i}
$$

and from (D.3.2)

$$
a_{02}>a_{01}
$$

Likewise, when the reffected wave is an expansion

$$
\frac{p_{21}}{p_{11}}<1
$$

and, since the expansion is moving to the left, while the flow is to the right

$$
\frac{u_{21}}{u_{11}}>1
$$

So from (D.3.1)

$$
M_{i}<M_{i}
$$

and from (D.3.2)

$$
a_{02}>a_{01}
$$

To summarize, for a decrease in sound speed across the interface, the transmitted shock is stronger than the incident shock and the reflected wave is a shock. For an increase in sound speed across the interface, the opposite is true. 


\section{Appendix $\mathbf{E}$}

\section{FLOWCHARTS AND COMPUTER PROGRAMS}

This appendix contains information on the algorithms used in the shock refraction calculations and provides listings of the main subprograms.

\section{E.1. Flowcharts}

The basic algorithms used for calculating regular and irregular refraction are presented in figures E.1(a) and E.1(b) respectively.

\section{E.2. Computer programs}

The following subprogram listings are provided:

INTPOL Subroutine for interpolating the value of $\omega$ given $M$, or viceversa, from a table of values obtained by integrating equation (2.3.11).

FAREA Function subprogram for evaluating $A$ using (A.B).

FCHI Function subprogram for evaluating $\chi$ using (2.4.3).

FLAMBDA Function subprogram for evaluating $\lambda$ using (2.1.21).

FMU Function subprogram for evaluating $\mu$ using (2.1.25).

FUPSILON Function subprogram for evaluating $v$ using (2.3.19).

DERIVM Subroutine for evaluating the derivative $d M / d a_{0}$ using (2.4.10).

MDQ Subroutine for numerically integrating equation (2.4.10).

TRT Subroutine for solving transcendental equations. 


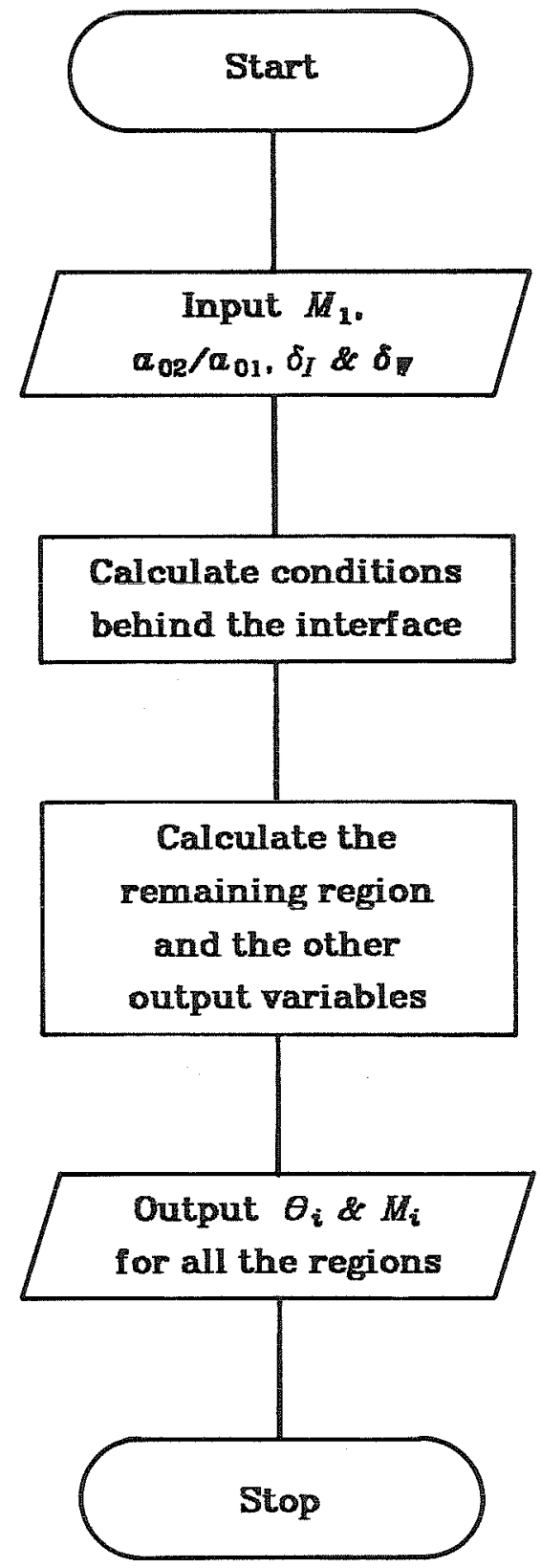

FIGURE E.1(a) Flowchart for regular refraction computations. 


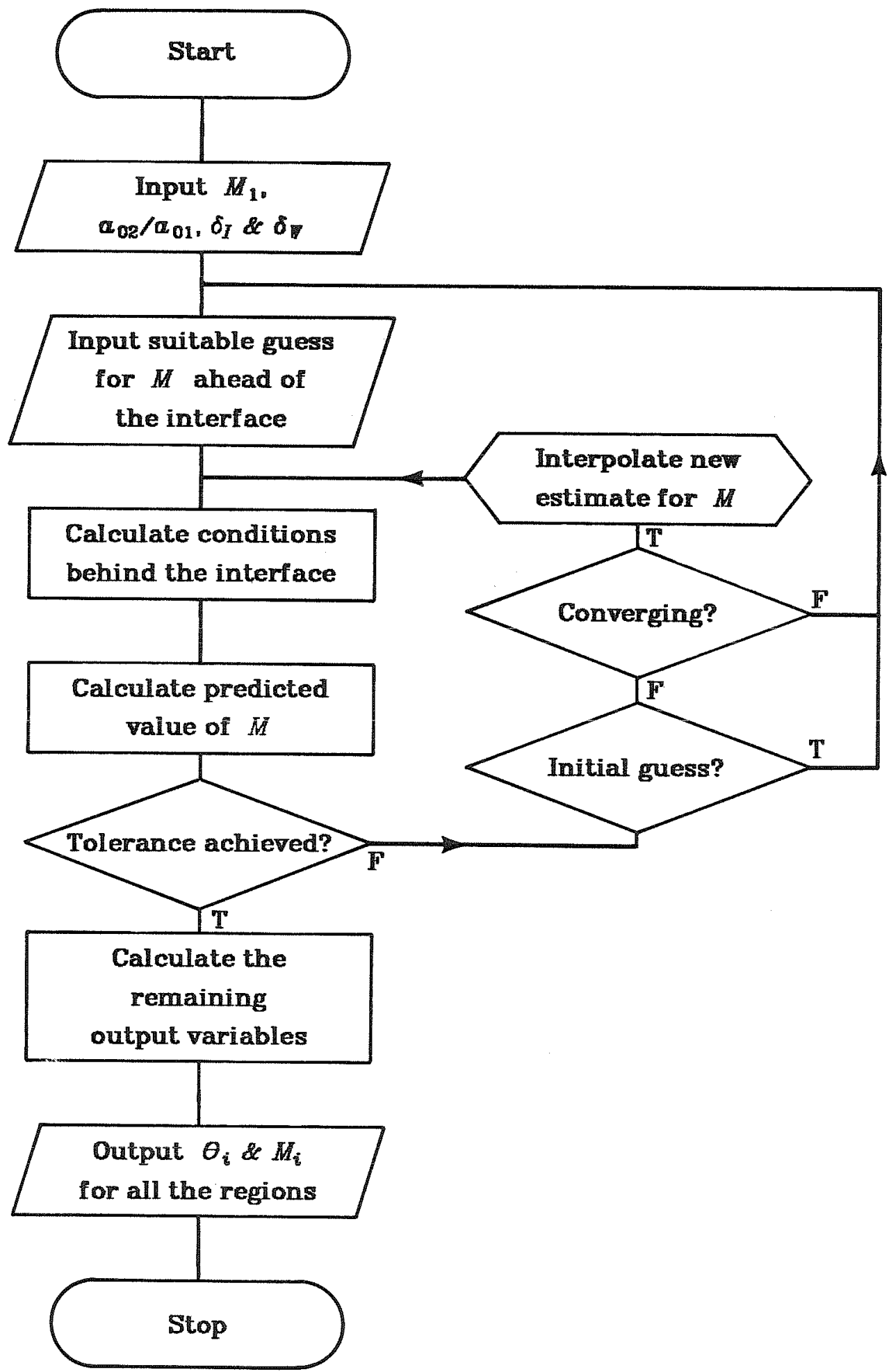

FIGURE E.1(b) Flowchart for irregular refraction computations. 
FILE: IACLMU.FOR

THIS FILE CONTAINS THE FOLLOWING SUBROUTINE AND FUNCTION SUBPROGRAMS:

- INTPOL(MNO,OMEGA,IT,*)

- FAREA(MNO)

- FCHI(TU,MU,ML)

- FLAMBDA(MNO)

- FMU(MNO)

- FUPSILON(MNO)

ANY CALLING PROGRAM MUST HAVE THE FOLLOWING OPENING STATEMENTS:

IMPLICIT REAL*8(A-H,L-Z)

COMMON /CONST/GAM,GAM1,GAM2

OPEN (UNIT $=11$, NAME $=$ 'CCWOMEGA.DRU',TYPE $={ }^{\prime} \mathrm{OLD}^{\prime}, \mathrm{READONLY}$,

1 ORGANIZATION='RELATIVE',ACCESS='DIRECT')

SUBROUTINE INTPOL(MNO,OMEGA,IT,*)

$\mathrm{C}$

$\mathrm{C}$

$\mathrm{C}$

$\mathrm{C}$

$\mathrm{C}$

$\mathrm{C}$

THIS SUBROUTINE PROVIDES THE VALUES OF MACH NUMBER AND OMEGA BY INTERPOLATION FOR ANY GIVEN MACH NUMBER (IT=1) OR OMEGA (IT=2).

IT USES DATA FROM THE UNFORMATTED DIRECT ACCESS FILE CCWOMEGA.DRU, (LOGICAL NUMBER 11).

IMPLICIT REAL* $(\mathrm{A}-\mathrm{H}, \mathrm{L}-\mathrm{Z})$

IF (IT.EQ.1.OR.IT.EQ.2) GO TO 1

GO TO 91

1 IREC $=135$

GO TO $(5,6)$ IT

5 IF (MNO.GE.1.ODO.AND.MNO.LT.1.000001DO) GO TO 81

IF (MNO.LT.1.ODO.OR.MNO.GT.100.0DO) GO TO 97

$\mathrm{X}=\mathrm{MNO}$

GO TO 10

6 IF (OMEGA.GE.O.ODO.AND.OMEGA.LT.2.828427232167634D-03) GO TO 82

IF (OMEGA.LT.O.ODO.OR.OMEGA.GT.1.165901164037424D+01) GO TO 99

$\mathrm{X}=\mathrm{OMEGA}$

$10 \quad \mathrm{IR}=\mathrm{IREC} / 2$

IRU $=$ IREC

$\operatorname{IRL}=1$

20 GO TO $(25,26)$ IT

25 READ(11'IR) XT,YT

GO TO 30

26 READ(11'IR) YT,XT

30 IF (X.GT.XT) GO TO 35 


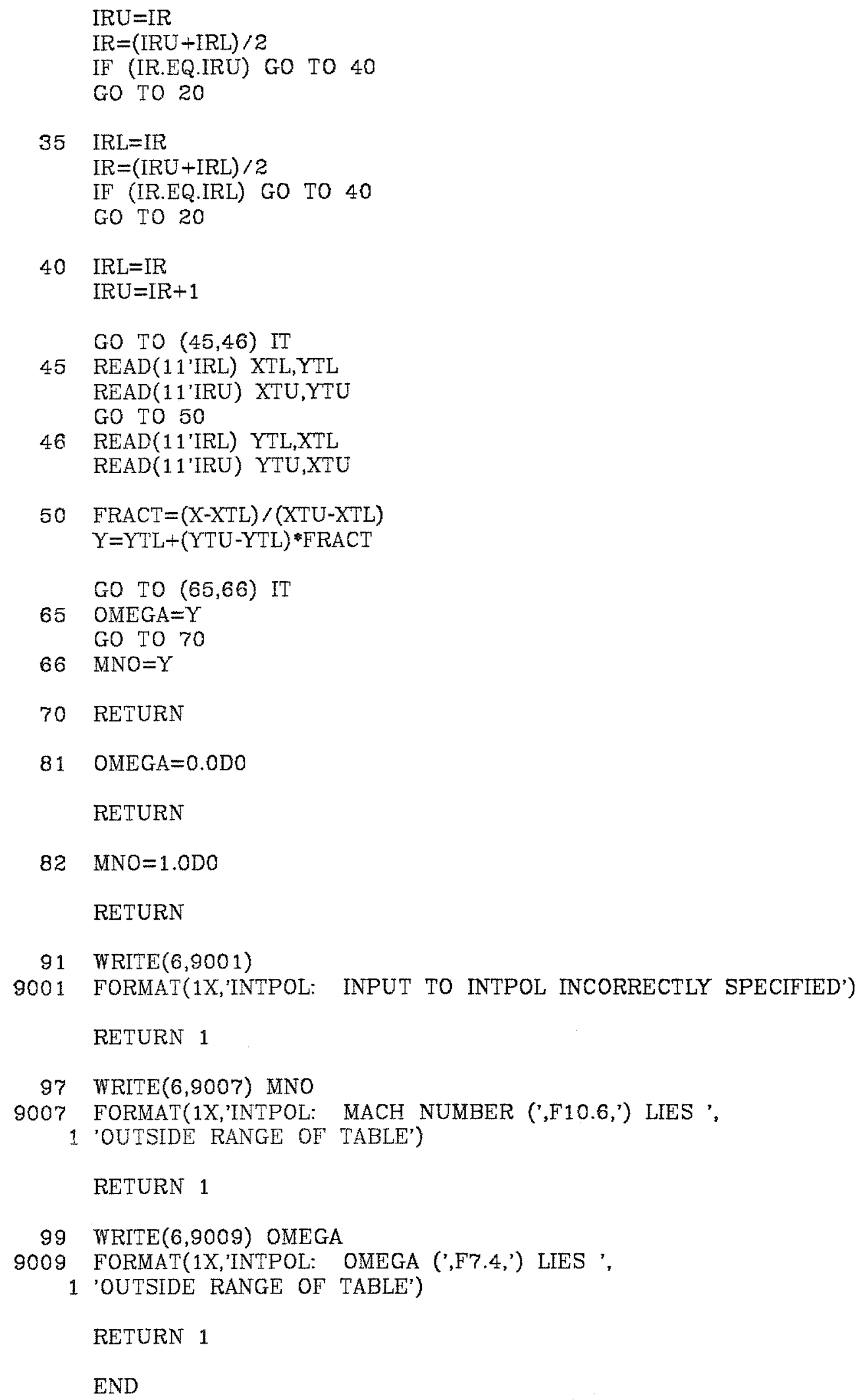


FUNCTION FAREA(MNO)

C

C THIS FUNCTION SUBPROGRAM EVALUATES THE RAY AREA FUNCTION IN

C TERMS OF THE MACH NUMBER. IT USES THE IMPROVED EXPRESSION.

$\mathrm{C}$

IMPLICIT REAL*8(A-H,L-Z)

COMMON /CONST/GAM,GAM1,GAM2

GAM3 = GAM1 / GAM2

SQGAM3 $=$ DSQRT $($ GAM3)

C1AREA $=$ DSQRT (2.0DO/GAM 1)

MNOSQ $=$ MNO $*$ MNO

$\mathrm{MU}=\mathrm{FMU}(\mathrm{MNO})$

FAREA $=($ MNO $/($ MNOSQ-1.ODO $) *((1.0 D O+M U) /(1.0 D O-M U)) *$

1 (MNOSQ-GAMB)**(-1.ODO/GAM)*

$2((\text { MU-SQGAM3 }) /(\text { MU +SQGAM3 }))^{*}$ DSQRT(GAM/2.ODO / GAM 1$) *$

3 DEXP(C1AREA*DATAN(MU/C1AREA))

RETURN

END

FUNCTION FCHI(TU,MU,ML)

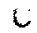

C THIS FUNCTION SUBPROGRAM EVALUATES THE SLOPE OF THE

C SHOCK-SHOCK LINE RELATIVE TO THE X-AXIS.

$\mathrm{C}$

IMPLICIT REAL*8(A-H,L-Z)

IF (ML.EQ.MU) GO TO 10

$\mathrm{AU}=\mathrm{FAREA}(\mathrm{MU})$

$A L=F A R E A(M L)$

FCHI $=$ TU+DATAN(AU /MU *DSQRT $((M L * M L-M U * M U) /$

$1(\mathrm{AU} * \mathrm{AU}-\mathrm{AL} * \mathrm{AL})))$

RETURN

$10 \quad \mathrm{FCHI}=\mathrm{TU}+\mathrm{FUPSILON}(\mathrm{MU})$

RETURN

END 
FUNCTION FLAMBDA(MNO)

C

C THIS FUNCTION SUBPROGRAM EVALUATES THE FUNCTION LAMBDA

C IN TERMS OF THE MACH NUMBER.

$\mathrm{C}$

IMPLICIT REAL*8(A-H,L-Z)

COMMON /CONST/GAM,GAM1,GAM2

$\mathrm{MU}=\mathrm{FMU}(\mathrm{MNO})$

FLAMBDA $=(1.0 \mathrm{DO}+2.0 \mathrm{DO} /(\mathrm{GAM}+1.0 \mathrm{DO}) *(1.0 \mathrm{DO}-\mathrm{MU} * \mathrm{MU}) / \mathrm{MU}) *$

1 (1.ODO+2.0DO* $\mathrm{MU}+1.0 \mathrm{DO} / \mathrm{MNO} / \mathrm{MNO})$

RETURN

END

FUNCTION FMU(MNO)

C

THIS FUNCTION SUBPROGRAM EVALUATES THE FUNCTION MU IN TERHS OF THE MACH NUMBER.

IMPLICIT REAL*8(A-H,L-Z)

COMMON /CONST/GAM, GAM1,GAM2

MNOSQ $=$ MNO $*$ MNO

MUSQ $=($ GAM $1 *$ MNOSQ $+2.0 D 0) /($ GAM $2 *$ MNOSQ-GAM1 $)$

$F M U=D S Q R T(M U S Q)$

RETURN

END

FUNCTION FUPSILON(MNO)

C

C THIS FUNCTION SUBPROGRAM CALCULATES THE SLOPE OF THE

C CHARACTERISTIC LINE RELATIVE TO THE FLOW DEFLECTION ANGLE.

$\mathrm{C}$

IMPLICIT REAL*8(A-H,L-Z)

FUPSILON=DATAN(DSQRT $(($ MNO + 1.ODO $) *($ MNO-1.0DO $) /$ FLAMBDA(MNO) $) /$ MNO $)$

RETURN

END 
SUBROUTINE DERIVM(AO,M,DMDAO, *)

$\mathrm{C}$

$\mathrm{C}$

THIS SUBROUTINE EVALUATES THE DERIVATIVE DMDAO

$\mathrm{C}$

IMPLICIT REAL*8(A-H,L-Z)

COMMON /CONST/GAM,GAM 1,GAM2

COMMON/DERI/CCSQ

IF (M.LE.1.ODO) GO TO 98

$\mathrm{MNO}=\mathrm{M}$

$\mathrm{MNOSQ}=\mathrm{MNO} * \mathrm{MNO}$

MNOSQ1 $=$ MNOSQ $-1.0 \mathrm{DO}$

$\mathrm{GG}=1.0 \mathrm{DO}+2.0 \mathrm{DO} * \mathrm{MNOSQ} 1 / \mathrm{DSQRT}(\mathrm{GAM} 2 * \mathrm{GAM} 1$ *MNOSQ*MNOSQ+

1 (4.0DO*GAM-GAM 1 GAM1)*MNOSQ-2.ODO*GAM1)

NU =DSQRT(MNOSQ1 $/$ FLAMBDA(MNO)) /MNO

$N U S Q=N U * N U$

$\mathrm{C} 1=\mathrm{AO} * \mathrm{M}$

$\mathrm{C} 1 \mathrm{SQ}=\mathrm{C} 1 * \mathrm{C} 1$

$\mathrm{C} 2 \mathrm{SQ}=\mathrm{CCSQ}-\mathrm{C} 1 \mathrm{SQ}$

C IF (C2SQ.LT.0.0DO) GO TO 99

$\mathrm{DMDAO}=\mathrm{NUSQ} * \mathrm{M} *(\mathrm{GG} * \mathrm{C} 2 \mathrm{SQ}-\mathrm{C} 1 \mathrm{SQ}) / \mathrm{AO} /(\mathrm{NUSQ} * \mathrm{C} 1 \mathrm{SQ}-\mathrm{C} 2 \mathrm{SQ})$

RETURN

\$8 RETURN 1

99 WRITE(6,9999) MNO,C2SQ

9999 FORMAT (1X,'DERIVM: M3 =',2PE15.6,' GIVES C2SQ =',1PE12.3) RETURN 1

END 
$\mathrm{C}$
$\mathrm{C}$
$\mathrm{C}$

FILE: MDQTRT.FOR

THIS FILE CONTAINS THE FOLLOWING SUBROUTINE SUBPROGRAMS:

- MDQ(FNCT,KK,X,Y,DYDX,DELX,EPS, *)

- SCHECK $(X, D Y D X, *)$

- RKG(FNCT,DX,X,Y,DYDX, *)

- AM(FNCT,DX,X,Y,DYDX, *)

- SAVE(ISR,X,Y,DYDX)

- TRT(FNCT,XST1,XST2,DX,EPS,ITMAX,IRTS,RTS,ITER,FRT, *)

THESE SUBROUTINES ARE SIMILAR TO THE CITLIB SUBROUTINES MODDEQ AND DTROOT RESPECTIVELY, EXCEPT FOR THE ERROR RETURN FACILITY.

SUBROUTINE MDQ(FNCT,KK,X,Y,DYDX,DELX,EPS,*)

IMPLICIT REAL*B(A-H,L-Z)

COMMON /INPUT/DUM1,DUM2,DUM3,DUM4,XSTART,XEND

COMMON /MDQAM/F(4),YP

COMMON /SLOPE/XEND1

IF (KK.LE.O.OR.KK.GE.3) GO TO 999

GO TO $(10,130)$, KK

C INITIAL CALL

10 CONTINUE

ISTEP $=4$

$\mathrm{DX}=\mathrm{DELX} / \mathrm{DFLOAT}$ (ISTEP)

DXMAX $=\mathrm{DELX}$

DXMIN $=$ DELX $/$ DFLOAT $(2 * 10)$

EUPPER $=$ EPS

ELOWER $=0.02 D O * E P S$

$\mathrm{IJ}=1$

CALL FNCT(X,Y,DYDX,\&99)

C WRITE $(6,8001) \mathrm{X}, \mathrm{Y}, \mathrm{DYDX}$

C8001 FORMAT(1X,'F:',3F 15.5)

$\mathrm{F}(\mathrm{IJ})=\mathrm{DYDX}$

$\mathrm{ISR}=1$

$\mathrm{KK}=2$

XEND $1=$ XEND $-1 . O D-6 *($ XEND-XSTART $)$

$X E N D I=X E N D-5.0 D O * D E L X$

RETURN

C SUBSEQUENT CALLS

130 CONTINUE

$\mathrm{XS}=\mathrm{X}$

$\mathrm{XE}=\mathrm{X}+\mathrm{DELX}$

IF (IJ.GE.4) GO TO 170 


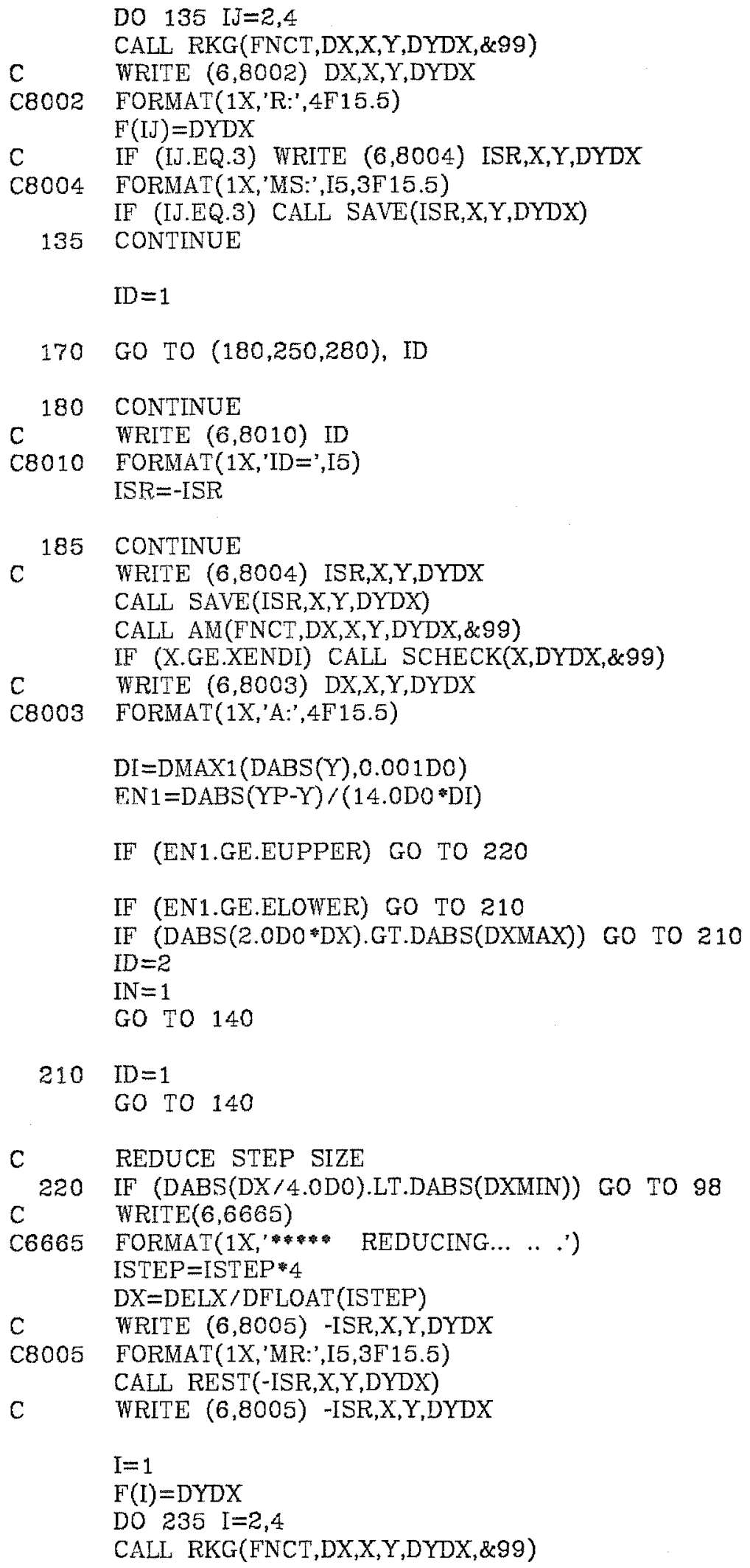




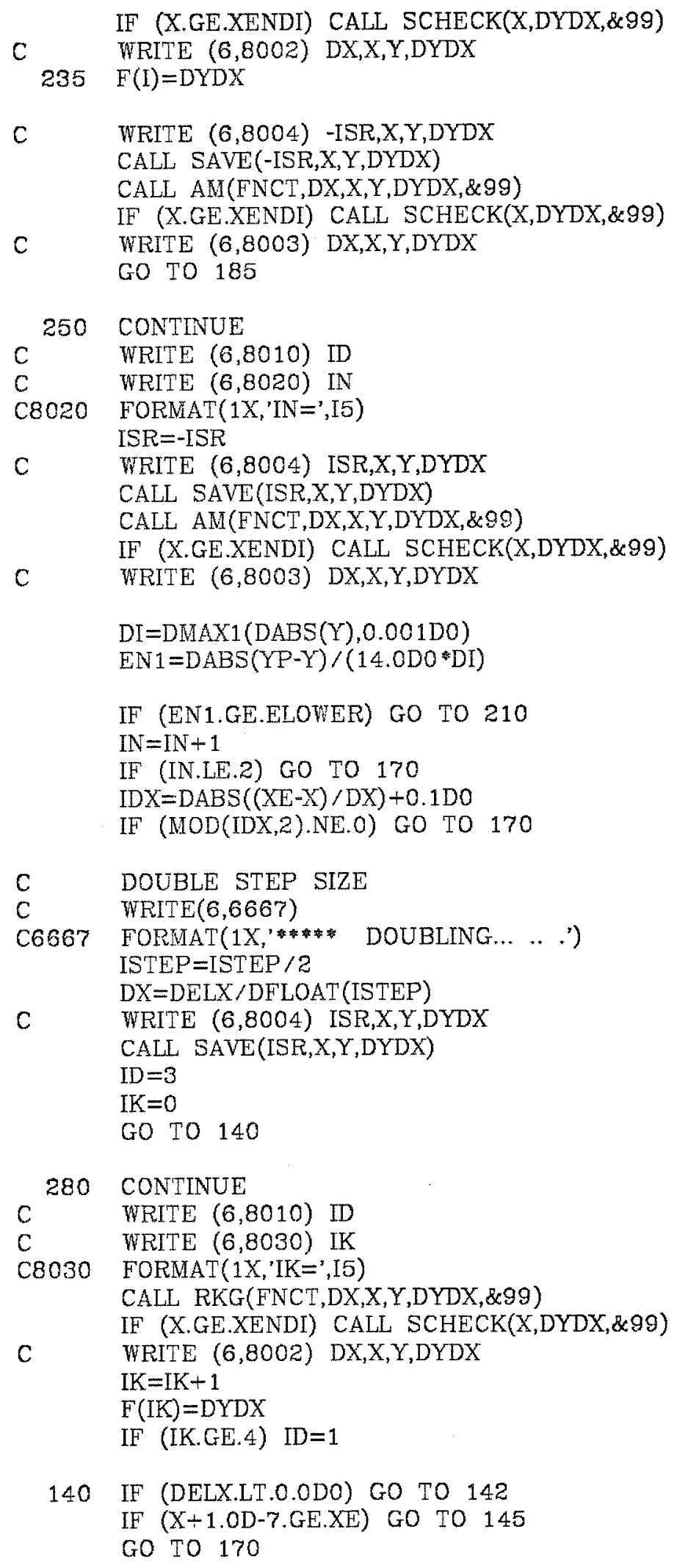




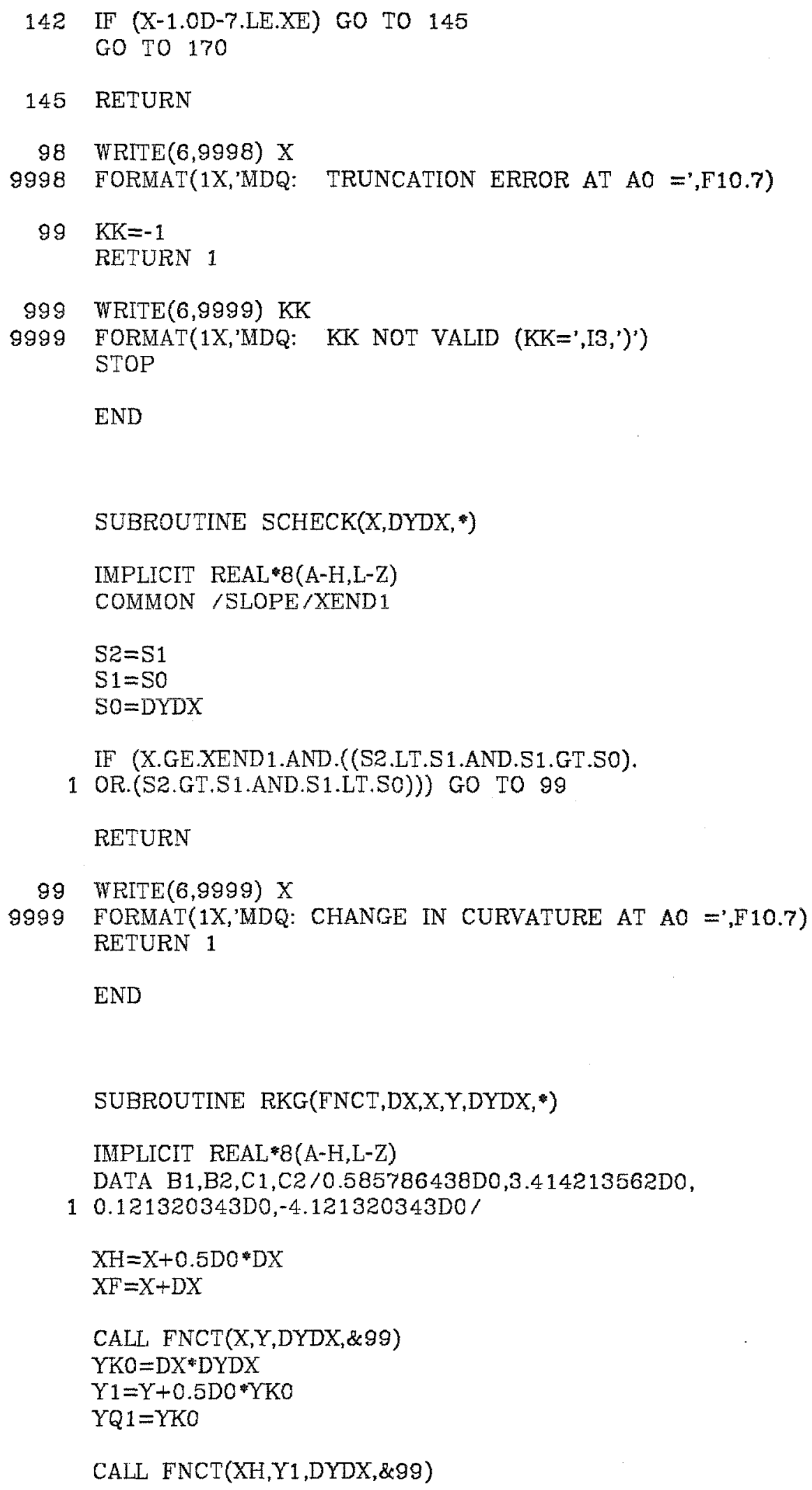


$\mathrm{YK} 1=\mathrm{DX} \cdot \mathrm{DYDX}$

$Y 2=Y 1+0.5 D 0 * B 1 *(Y K 1-Y Q 1)$

$\mathrm{YQ2}=\mathrm{B} 1 * \mathrm{YK} 1+\mathrm{C} 1 * \mathrm{YQ} 1$

CALL FNCT(XH,Y2,DYDX,\&99)

$\mathrm{YKZ}=\mathrm{DX} * \mathrm{DYDX}$

$\mathrm{Y} 3=\mathrm{YZ}+0.5 \mathrm{DO} * \mathrm{BZ} *(\mathrm{YKZ}-\mathrm{YQZ})$

$\mathrm{YQ3}=\mathrm{B} 2 * \mathrm{YK} 2+\mathrm{C2} * \mathrm{YQ2}$

CALL FNCT(XF,Y3,DYDX,\&99)

$\mathrm{YK} 3=\mathrm{DX} * \mathrm{DYDX}$

$Y_{4}=Y 3+(0.5 D 0 * Y K 3-Y Q 3) / 3.0 D 0$

$\mathrm{X}=\mathrm{XF}$

$Y=Y 4$

CALL FNCT(X,Y,DYDX,\&99)

RETURN

99 RETURN 1

END

SUBROUTINE AM(FNCT,DX,X,Y,DYDX, $\left.{ }^{*}\right)$

IMPLICIT REAL *8(A-H,L-Z)

COMMON /MDQAM/ $\mathrm{F}(4), \mathrm{YP}$

$\mathrm{XF}=\mathrm{X}+\mathrm{DX}$

$\mathrm{YP}=\mathrm{Y}+(\mathrm{DX} / 24.0 \mathrm{DO}) *(55.0 \mathrm{DO} * \mathrm{~F}(4)-59.0 \mathrm{DO} * \mathrm{~F}(3)+37.0 \mathrm{DO} * \mathrm{~F}(2)-$

$19.0 \mathrm{DO} * \mathrm{~F}(1))$

CALL FNCT(XF,YP,DYDX,\&99)

$\mathrm{YC}=\mathrm{Y}+(\mathrm{DX} / 24.0 \mathrm{DO}) *(9.0 \mathrm{DO} * \mathrm{DYDX}+19.0 \mathrm{DO} * \mathrm{~F}(4)-5.0 \mathrm{DO} * \mathrm{~F}(3)+$

$1 F(2))$

$\mathrm{X}=\mathrm{XF}$

$\mathrm{Y}=\mathrm{YC}$

CALL FNCT(X,Y,DYDX,\&99)

DO $10 \mathrm{I}=1,3$

$10 \quad F(I)=F(I+1)$

$F(4)=D Y D X$

RETURN

99 RETURN 1

END 
SUBROUTINE SAVE(ISR,X,Y,DYDX)

IMPLICIT REAL *8(A-H,L-Z)

IF (ISR.LT.0) GO TO 25

$\mathrm{XA}=\mathrm{X}$

$\mathrm{YA}=\mathrm{Y}$

DYDXA=DYDX

RETURN

$25 \quad \mathrm{XB}=\mathrm{X}$

$\mathrm{YB}=\mathrm{Y}$

DYDXB $=$ DYDX

RETURN

C RESTORE

ENTRY REST(ISR,X,Y,DYDX)

IF (ISR.LT.0) GO TO 75

$\mathrm{X}=\mathrm{XA}$

$Y=Y A$

DYDX $=$ DYDXA

RETURN

$75 \mathrm{X}=\mathrm{XB}$

$\mathrm{Y}=\mathrm{YB}$

$D Y D X=D Y D X B$

RETURN

END 
SUBROUTINE TRT(FNCT,XST1,XST2,DX,EPS,ITMAX,IRTS,RTS,ITER,FRT,*)

IMPLICIT REAL*8(A-H,L-Z)

DIMENSION RTS(1),ITER(1),FRT(1)

C INITIALIZE

IF (XST1-XST2) 4,99,6

$4 \mathrm{XSTART}=\mathrm{XST} 1$

XSTOP $=$ XST2

GO TO 10

$6 \mathrm{XSTART}=\mathrm{XST} 2$

$\mathrm{XSTOP}=\mathrm{XST} 1$

10 IF (DX.LE.O.ODO.OR.EPS.LE.O.ODO) GO TO 99

$\mathrm{II}=1$

$\mathrm{XL}=\mathrm{XSTART}$

$\mathrm{XU}=\mathrm{XI}+\mathrm{DX}$

$F X L=F N C T(X L)$

$11 \mathrm{FXU}=\mathrm{FNCT}(\mathrm{XU})$

C CHECK FOR SIGN CHANGE OR IDENTICAL ZERO

IF (FXL*FXU) 14,15,16

$16 \mathrm{XL}=\mathrm{XU}$

IF (XL.GE.XSTOP) GO TO 100

$\mathrm{XU}=\mathrm{XU}+\mathrm{DX}$

IF (XU.GT.XSTOP) XU=XSTOP

$\mathrm{FXL}=\mathrm{FXU}$

GO TO 11

15 ITER(II) $=0$

IF (FXL.EQ.0.0D0) RTS(II)=XL

IF (FXU.EQ.0.0DO) RTS(II) $=\mathrm{XU}$

FRT(II) $=0.0 \mathrm{DO}$

FXU2 $=$ FXU

$\mathrm{XU} 2=\mathrm{XU}$

GO TO 50

14 FXU2 = FXU

$\mathrm{XU} 2=\mathrm{XU}$

C INTERPOLATION LOOP

DO $29 \mathrm{~J}=1$,ITMAX

$\mathrm{J} J=\mathrm{J}$

$\mathrm{XRT}=\mathrm{XU}-\mathrm{FXU} *(\mathrm{XU}-\mathrm{XL}) /(\mathrm{FXU}-\mathrm{FXL})$

FTEMP $=$ FNCT $(X R T)$

IF (DABS(FTEMP).LE.EPS) GO TO 30

IF (XRT.EQ.XU) GO TO 40

IF (FTEMP*FXU.LT.O.ODO) GO TO 24

$F X L=F X L^{*} 0.5 D O$

GO TO 26

$24 \mathrm{XL}=\mathrm{XU}$

$F X L=F X U$ 


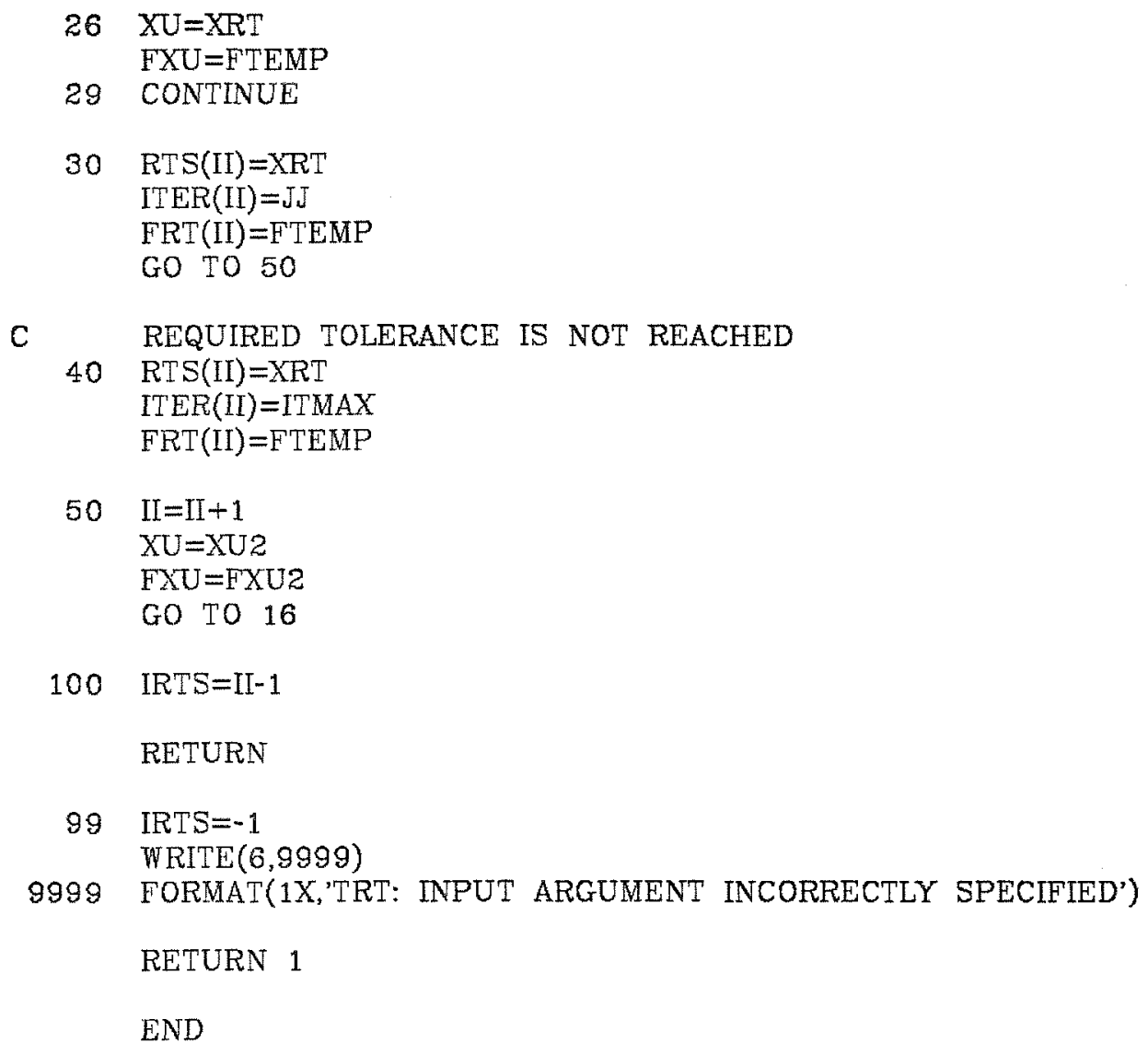

